\title{
Synthesis of (+)-Omphadiol and (+)-Pyxidatol C
}

Gowrisankar Parthasarathy, Ulrike Eggert, and Markus Kalesse*

Institute for Organic Chemistry and Centre for Biomolecular Drug Research, Leibniz Universität Hannover, Schneiderberg 1B, 30167 Hannover, Germany

E-mail: Markus.Kalesse@oci.uni-hannover.de

\section{Supplementary Information}

CONTENTS:

Page

1. General

02-02

2. Experimental procedures for (+)-omphadiol

03-10

3. Comparison table of (+)-omphadiol

4. References

5. Experimental procedures for (+)-pyxidatol C

$13-18$

6. Comparison table of (+)-pyxidatol C

7. References

$20-20$

8. Spectroscopic data of (+)-omphadiol

21-34

9. Spectroscopic data of (+)-pyxidatol C 


\section{Experimental section}

General:

Unless otherwise indicated, all reactions were carried out under a positive pressure of nitrogen and with ovendried glassware using standard Schlenk techniques. Infrared spectra (IR) were recorded on a Perkin-Elmer FTIR SPECTRUM ONE spectrometer (diamond ATR Golder Gate sampling). Proton nuclear magnetic resonance ( $\left({ }^{1} \mathrm{H}\right.$ NMR) spectra were recorded on Bruker AMX 300 or 400 . Chemical shifts are reported in parts per million $(\mathrm{ppm})$ relative to internal standard (tetramethylsilane, $\delta_{\mathrm{H}}=0.00 ; \mathrm{CDCl}_{3}, \delta_{\mathrm{H}}=7.26$; acetone- ${ }_{\mathrm{d} 6}, \delta_{\mathrm{H}}=$ 2.05 and $\left.\mathrm{C}_{6} \mathrm{D}_{6}, \delta_{\mathrm{H}}=7.16\right)$. Data are presented as follows: chemical shift $(\delta, \mathrm{ppm})$, integration, multiplicity $(\mathrm{s}=$ singlet, $\mathrm{d}=$ doublet, $\mathrm{t}=$ triplet, $\mathrm{q}=$ quadruplet, $\mathrm{m}=$ multiplet, $\mathrm{br}=$ broad), coupling constant (reported in $\mathrm{Hz}$ ), assignment. Carbon magnetic resonance $\left({ }^{13} \mathrm{C}\right.$ NMR) spectra were recorded on Bruker AMX 300 or 400 . Chemical shifts are reported in parts per million $(\mathrm{ppm})$ relative to internal standard (tetramethylsilane, $\delta_{\mathrm{C}}=$ 0.00; $\mathrm{CDCl}_{3}, \delta_{\mathrm{C}}=77.00$ ). Electron impact (EI) mass spectra were obtained using Varian CH-4 or SM-1 instruments operating at $40 \mathrm{eV}$ and electrospray (ESI) spectra using an Applied Biosystems API 150EX LC/MS system. Product purification by flash column chromatography was performed using Brunschwig silica gel $60 \AA$ (32-63 mesh). Analytical thin layer chromatography (TLC) was carried out using Merck commercial aluminium sheets coated $\left(0.2 \mathrm{~mm}\right.$ layer thickness) with Kieselgel $60 \mathrm{~F}_{254}$, with visualization by ultraviolet. THF was freshly distilled from Na. $\mathrm{CH}_{2} \mathrm{Cl}_{2}$ was freshly distilled from $\mathrm{CaH}_{2}$. Diethyl ether, $n$-hexane, toluene were purified on $\mathrm{Al}_{2} \mathrm{O}_{3}$ columns. Diisopropylamine was distilled over sodium hydride prior to use. Cyclohexane and ethyl acetate for flash chromatography are used without further purification. 


\section{3-Hydroxy-2,2-dimethylpropyl pivalate (A)}

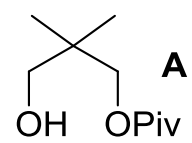

To a solution of 2,2-methyl-propane-1,3-diol $(2.5 \mathrm{~g}, 24.05 \mathrm{mmol})$ in $\mathrm{CH}_{2} \mathrm{Cl}_{2}(125 \mathrm{~mL})$ at room temperature was added triethylamine $(5 \mathrm{~mL}, 36.01 \mathrm{mmol})$ and pivaloyl chloride $(3.1 \mathrm{~mL}, 25.21 \mathrm{mmol})$ at room temperature. The resultant mixture was stirred at room temperature for overnight and quenched with brine. The layers were separated and the organic layer was dried $\left(\mathrm{Na}_{2} \mathrm{SO}_{4}\right)$, concentrated under vacuum and silica gel flash chromatography (hexanes/ether, 3:1) to give 3-hydroxy-2,2-dimethylpropyl pivalate $\mathbf{A}(3.6 \mathrm{~g}, 80 \%)$ as a colorless oil. ${ }^{1} \mathbf{H}$ NMR (200 MHz, $\left.\mathbf{C D C l}_{\mathbf{3}}\right) \boldsymbol{\delta} 3.90$ (s, 2H), 3.26 (s, 2H), 1.20 (s, 9H), 0.91 (s, 6H); ${ }^{13} \mathbf{C}$ NMR (101 MHz, $\left.\mathbf{C D C l}_{\mathbf{3}}\right) \boldsymbol{\delta}$ 179.2, 69.1, 68.1, 38.9, 36.6, 27.2, 21.4.

\section{2,2-Dimethyl-3-oxopropyl pivalate (10)}

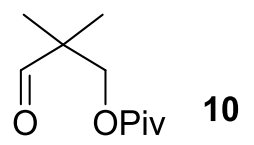

To a solution of oxalyl chloride $(2.0 \mathrm{~mL}, 22.34 \mathrm{mmol})$ in $\mathrm{CH}_{2} \mathrm{Cl}_{2}(40 \mathrm{~mL})$ was added DMSO $(2.6 \mathrm{~mL}, 36.70$ mmol) at $-78{ }^{\circ} \mathrm{C}$ over a period of 5 mins. After $15 \mathrm{~min}$, a solution of alcohol $\mathbf{A}(3.0 \mathrm{~g}, 15.96 \mathrm{mmol})$ in $\mathrm{CH}_{2} \mathrm{Cl}_{2}$ $(15 \mathrm{~mL})$ was added into the above reaction mixture at $-78{ }^{\circ} \mathrm{C}$ and allowed to stir for additional $1 \mathrm{~h}$ and quenched with $\mathrm{NEt}_{3}(11.10 \mathrm{~mL}, 79.8 \mathrm{mmol})$ at $-78{ }^{\circ} \mathrm{C}$. The turbid solution was allowed to attain room temperature over a period of $1 \mathrm{~h}$. The reaction mixture was diluted with water $(50 \mathrm{~mL})$ and extracted with diethyl ether $(2 \times 20 \mathrm{~mL})$ followed by brine $(20 \mathrm{~mL})$ then dried over $\left(\mathrm{Na}_{2} \mathrm{SO}_{4}\right)$. The obtained crude aldehyde $\mathbf{1 0}$ was purified by flash chromatography (hexanes/ethyl acetate 5:1) to yield pure compound $\mathbf{1 0}(2.6 \mathrm{~g}, 82 \%) .{ }^{1} \mathbf{H}$

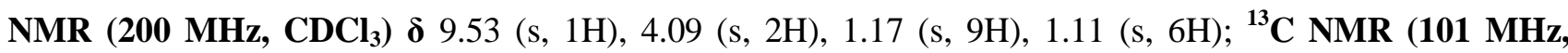
$\left.\mathbf{C D C l}_{3}\right) \boldsymbol{\delta} 203.4,178.0,67.7,46.5,38.8,27.0,18.8$.

(R)-3-hydroxy-2,2-dimethyl-3-((2S,3S,3aS,4S,7R,7aS)-1-oxo-3-(prop-1-en-2-yl)-2,3,3a,4,7,7a-hexahydro1H-4,7-methanoinden-2-yl)propyl pivalate

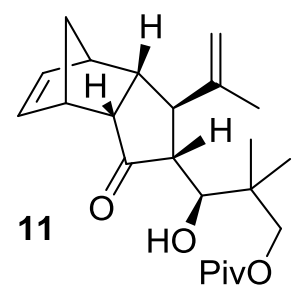

tert-BuLi (28.8 $\mathrm{mL}, 1.6 \mathrm{M}$ in pentane, $46.1 \mathrm{mmol})$ was added to a solution of 2-bromopropene ( $2 \mathrm{~mL}, 21.9$ mmol) in anhydrous ether $(120 \mathrm{~mL})$ at $-78^{\circ} \mathrm{C}$. The resultant solution was stirred at $-78{ }^{\circ} \mathrm{C}$ for $30 \mathrm{~min}$. and then warmed to $0{ }^{\circ} \mathrm{C}$ for $30 \mathrm{~min}$. The above prepared solution was transferred into a suspension of $\mathrm{CuI}(2.1 \mathrm{~g}, 10.96$ 
$\mathrm{mmol})$ in ether $(10 \mathrm{~mL})$ at $-78{ }^{\circ} \mathrm{C}$ and warmed to $\mathrm{rt}$ for $10 \mathrm{~min}$. The resultant black mixture was cooled to -78 ${ }^{\circ} \mathrm{C}$ and treated with a solution of (-)-KDP enone 9 (1.6 g, $\left.10.96 \mathrm{mmol}\right)$ in ether $(2 \mathrm{~mL})$. The resultant brown mixture was warmed to $-40{ }^{\circ} \mathrm{C}$ for $2 \mathrm{~h}$ and then aldehyde $\mathbf{A}(3,3 \mathrm{~g}, 17.53 \mathrm{mmol})$ was added at $-78{ }^{\circ} \mathrm{C}$ and stirred at $-30{ }^{\circ} \mathrm{C}$ for $2 \mathrm{~h}$, then at $-15{ }^{\circ} \mathrm{C}$ for another $1 \mathrm{~h}$. The reaction was quenched with a saturated aqueous $\mathrm{NH}_{4} \mathrm{Cl}$ solution $(10 \mathrm{~mL})$ at $-78{ }^{\circ} \mathrm{C}$ and the aqueous layer was extracted with ether $(3 \times 20 \mathrm{~mL})$. The combined ether layers were washed with a saturated aqueous $\mathrm{NaCl}$ solution and dried over $\mathrm{Na}_{2} \mathrm{SO}_{4}$. Silica gel flash chromatography to afford a pure aldol 11 as white solid (3.64 g, 88\%). ${ }^{1} \mathbf{H}$ NMR (400 $\left.\mathbf{M H z}, \mathbf{C D C l}_{3}\right) \delta$ 6.23 $(\mathrm{dd}, J=5.7,2.9 \mathrm{~Hz}, 1 \mathrm{H}), 6.15(\mathrm{dd}, J=5.7,3.0 \mathrm{~Hz}, 1 \mathrm{H}), 4.91-4.87(\mathrm{~m}, 1 \mathrm{H}), 4.83(\mathrm{~s}, 1 \mathrm{H}), 3.96(\mathrm{~d}, J=10.9$ $\mathrm{Hz}, 1 \mathrm{H}), 3.80(\mathrm{~d}, J=10.9 \mathrm{~Hz}, 1 \mathrm{H}), 3.24$ (d, $J=1.1 \mathrm{~Hz}, 2 \mathrm{H}), 3.14$ (bs, $1 \mathrm{H}), 3.05$ (ddd, $J=10.8,4.3,2.3 \mathrm{~Hz}$, 1H), 2.99 (bs, 1H), $2.90-2.82(\mathrm{~m}, 1 \mathrm{H}), 2.71(\mathrm{dd}, J=13.0,2.4 \mathrm{~Hz}, 1 \mathrm{H}), 2.41(\mathrm{dd}, J=13.0,8.1 \mathrm{~Hz}, 1 \mathrm{H}), 1.80$ $(\mathrm{s}, 3 \mathrm{H}), 1.65(\mathrm{dt}, J=8.3,1.7 \mathrm{~Hz}, 1 \mathrm{H}), 1.46(\mathrm{~d}, J=8.4 \mathrm{~Hz}, 1 \mathrm{H}), 1.18(\mathrm{~s}, 9 \mathrm{H}), 0.88(\mathrm{~s}, 3 \mathrm{H}), 0.86(\mathrm{~s}, 3 \mathrm{H}) ;{ }^{13} \mathbf{C}$

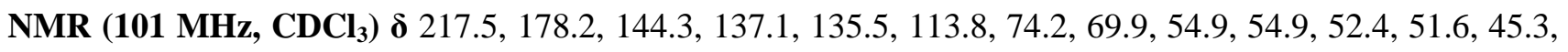
44.0, 43.6, 39.0, 38.9, 27.2, 22.2, 20.6, 18.6; IR (ATR, neat) $\boldsymbol{v}_{\max } 3528,2964,1720,1479,1285,1174,883$ $\mathrm{cm}^{-1}$; HRMS (ESI-TOF): calcd. for $\mathrm{C}_{23} \mathrm{H}_{35} \mathrm{O}_{4}(\mathrm{M}+1)^{+} \mathrm{m} / \mathrm{z} 375.2538$ found $\mathrm{m} / \mathrm{z} 375.2535$. [a] ${ }^{\mathbf{2 0}}{ }_{\mathbf{D}}+12.8(c$ $\left.0.81, \mathrm{CHCl}_{3}\right)$.

\section{(E)-2,2-dimethyl-3-((3R,3aS,4S,7R,7aS)-1-oxo-3-(prop-1-en-2-yl)-1,3,3a,4,7,7a-hexahydro-2H-4,7-} methanoinden-2-ylidene)propyl pivalate

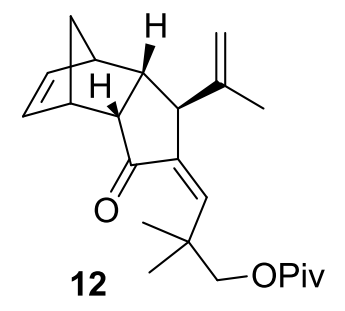

To a solution of Keto1 11 (3.5 g, $9.36 \mathrm{mmol})$ in THF $(320 \mathrm{~mL})$ was added methyl chlorosulfonate $(2.2 \mathrm{~mL}$, $28.07 \mathrm{mmol})$ and DMAP $(5.71 \mathrm{~g}, 46.8 \mathrm{mmol})$ at room temperature. The resultant white suspension was heated to reflux for 4 days and concentrated in vacuo to remove the volatiles. The reaction mixture was diluted with water $(15 \mathrm{~mL})$ and extracted with diethyl ether $(150 \mathrm{~mL})$. The combined organic layer was washed with water $(10 \mathrm{~mL})$, brine $(10 \mathrm{~mL})$, dried over $\mathrm{Na}_{2} \mathrm{SO}_{4}$, and concentrated. The obtained crude product was purified by chromatography on silica gel (hexanes/EtOAc, 5:1) to afford 3.1 g (93\%) of enone $\mathbf{1 2}$ as white solid. ${ }^{\mathbf{1}} \mathbf{H}$ NMR $\left(400 \mathrm{MHz}, \mathbf{C D C l}_{3}\right) \boldsymbol{\delta} 6.40(\mathrm{~d}, J=2.1 \mathrm{~Hz}, 1 \mathrm{H}), 6.00(\mathrm{dd}, J=5.6,2.9 \mathrm{~Hz}, 1 \mathrm{H}), 5.95(\mathrm{dd}, J=5.6,2.9 \mathrm{~Hz}, 1 \mathrm{H})$, $4.72-4.70(\mathrm{~m}, 1 \mathrm{H}), 4.59(\mathrm{~d}, J=0.7 \mathrm{~Hz}, 1 \mathrm{H}), 3.89(\mathrm{~d}, J=10.6 \mathrm{~Hz}, 1 \mathrm{H}), 3.86(\mathrm{~d}, J=10.6 \mathrm{~Hz}, 1 \mathrm{H}), 3.27-3.22$ (m, 1H), $3.16(\mathrm{~s}, 1 \mathrm{H}), 3.08(\mathrm{bs}, 1 \mathrm{H}), 2.95(\mathrm{dd}, J=8.8,4.8 \mathrm{~Hz}, 1 \mathrm{H}), 2.58$ (ddd, $J=8.8,4.1,1.6 \mathrm{~Hz}, 1 \mathrm{H}), 1.75$ (s, 3H), $1.50(\mathrm{dt}, J=8.3,1.7 \mathrm{~Hz}, 1 \mathrm{H}), 1.41(\mathrm{~d}, J=8.4 \mathrm{~Hz}, 1 \mathrm{H}), 1.19(\mathrm{~s}, 9 \mathrm{H}), 1.12(\mathrm{~s}, 3 \mathrm{H}), 1.04(\mathrm{~s}, 3 \mathrm{H}) ;{ }^{13} \mathbf{C}$

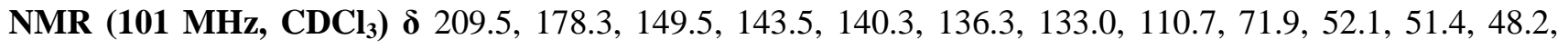
47.6, 47.6, 45.6, 38.9, 37.5, 27.2, 23.6, 23.3, 21.3; IR (ATR, neat) $\boldsymbol{v}_{\max } 2979,2926,1727,1708,1627,1365$, 
1291, 1234, 1173, $889 \mathrm{~cm}^{-1}$; HRMS (ESI-TOF): calcd. for $\mathrm{C}_{23} \mathrm{H}_{32} \mathrm{O}_{3} \mathrm{Na}(\mathrm{M}+\mathrm{Na})^{+} \mathrm{m} / \mathrm{z} 379.2251$ found $\mathrm{m} / \mathrm{z}$ $379.2249 ;[\boldsymbol{\alpha}]^{\mathbf{2 0}}{ }_{\mathbf{D}}-202.3\left(\right.$ c $\left.1.04, \mathrm{CHCl}_{3}\right)$.

(2R,3S,3aS,4S,7R,7aS)-2-(3-hydroxy-2,2-dimethylpropyl)-3-(prop-1-en-2-yl)-2,3,3a,4,7,7a-hexahydro-1H-

\section{4,7-methanoinden-1-one}

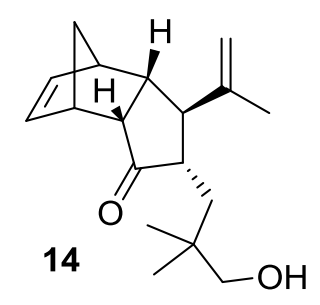

Lithium metal pieces $\left(250 \mathrm{mg}, 35.4 \mathrm{mmol}\right.$ ) were added to an anhydrous liquid $\mathrm{NH}_{3}$ (about $150 \mathrm{~mL}$ ) at $-78{ }^{\circ} \mathrm{C}$ under nitrogen atmosphere. The resultant blue solution was treated with a solution of $\mathbf{1 2}(1.6 \mathrm{~g}, 5.06 \mathrm{mmol})$ in THF (100 mL) at $-78{ }^{\circ} \mathrm{C}$. The reaction mixture was allowed to stir for $1 \mathrm{~h}$ at $-78{ }^{\circ} \mathrm{C}$, then quenched with tert. $\mathrm{BuOH}(50 \mathrm{~mL})$ followed by $\mathrm{MeOH}(10 \mathrm{~mL})$ at the same temperature, then allowed to attain room temperature. The excess $\mathrm{NH}_{3}$ was evaporated under the stream of nitrogen flow and allowed to stir at room temperature for another $2 \mathrm{~h}$. The resultant white suspension was treated with neat methyl salicylate $(4.5 \mathrm{~mL}$, $35.4 \mathrm{mmol}$ ) and allowed to stir for $30 \mathrm{mins}$ at the same temperature. The reaction was diluted with saturated aqueous $\mathrm{NH}_{4} \mathrm{Cl}(20 \mathrm{~mL})$ and extracted with EtOAc $(3 \mathrm{X} 40 \mathrm{~mL})$. The combined organic layers were dried $\mathrm{Na}_{2} \mathrm{SO}_{4}$ and concentration in vacuo gave crude 14. Flash chromatography on silica gel (hexanes:EtOAc, 4:1) to afford $960 \mathrm{mg}(80 \%)$ of hydroxylketone $\mathbf{1 4}$ as single diastereomer. ${ }^{1} \mathbf{H}$ NMR (400 $\left.\mathbf{~ M H z}, \mathbf{C D C l}_{3}\right) \boldsymbol{\delta} 6.22$ $(\mathrm{dd}, J=5.7,2.5 \mathrm{~Hz}, 1 \mathrm{H}), 6.08(\mathrm{dd}, J=5.8,3.0 \mathrm{~Hz}, 1 \mathrm{H}), 4.90-4.87(\mathrm{~m}, 1 \mathrm{H}), 4.78(\mathrm{~s}, 1 \mathrm{H}), 4.12(\mathrm{dd}, J=10.3$, $4.6 \mathrm{~Hz}, 1 \mathrm{H}), 3.19-3.11(\mathrm{~m}, 3 \mathrm{H}), 3.07-3.02(\mathrm{~m}, 1 \mathrm{H}), 2.98(\mathrm{bs}, 1 \mathrm{H}), 2.92-2.84(\mathrm{~m}, 1 \mathrm{H}), 2.45-2.36(\mathrm{~m}, 1 \mathrm{H})$, $1.88-1.83(\mathrm{~m}, 1 \mathrm{H}), 1.82(\mathrm{~s}, 3 \mathrm{H}), 1.71-1.62(\mathrm{~m}, 2 \mathrm{H}), 1.49(\mathrm{~d}, J=8.3 \mathrm{~Hz}, 1 \mathrm{H}), 0.94(\mathrm{~s}, 3 \mathrm{H}), 0.75(\mathrm{~d}, J=14.6$ $\mathrm{Hz}, 1 \mathrm{H}), 0.66(\mathrm{~s}, 3 \mathrm{H}) ;{ }^{13} \mathbf{C}$ NMR (101 MHz, $\left.\mathbf{C D C l}_{3}\right) \boldsymbol{\delta} 220.7,144.4,137.3,135.4,113.2,69.4,54.4,54.2$, 52.5, 51.9, 45.2, 43.9, 43.8, 35.2, 34.2, 26.6, 23.2, 18.6; IR (ATR, neat) $\boldsymbol{v}_{\max } 3463,2954,2868,1721,1449$, 1048, $894 \mathrm{~cm}^{-1}$; HRMS (ESI-TOF): calcd. for $\mathrm{C}_{18} \mathrm{H}_{26} \mathrm{O}_{2} \mathrm{Na}(\mathrm{M}+\mathrm{Na})^{+} \mathrm{m} / \mathrm{z} 297.1829$ found $\mathrm{m} / \mathrm{z}$ 297.1831; $[\boldsymbol{\alpha}]^{\mathbf{2 0}}{ }_{\mathbf{D}}-2.0\left(c 0.85, \mathrm{CHCl}_{3}\right)$. 


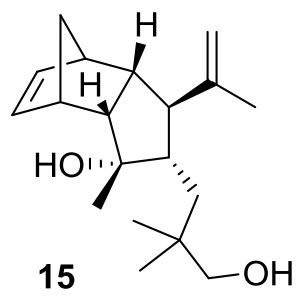

To a flask was added $\mathrm{CeCl}_{3}-7 \mathrm{H}_{2} \mathrm{O}$ and heated at $100{ }^{\circ} \mathrm{C}$ for $1 \mathrm{~h}$, then at $130{ }^{\circ} \mathrm{C}$ for additional $1 \mathrm{~h}$ without stirring under high vacuum $(0.1 \mathrm{~mm} / \mathrm{Hg})$. Later, allowed to attain room temperature and flushed with Nitrogen. The obtained anhydrous $\mathrm{CeCl}_{3}$ was treated with anhydrous $\mathrm{THF}(40 \mathrm{~mL})$ at $0{ }^{\circ} \mathrm{C}$ and stirred at $\mathrm{rt}$ for $3 \mathrm{~h}$. The above suspension was cooled to $-78{ }^{\circ} \mathrm{C}$ and treated with $\mathrm{MeLi}(10.6 \mathrm{~mL}, 16.9 \mathrm{mmol})$, and the resultant yellow color suspension was stirred for $30 \mathrm{mint}$ at the same temperature. A solution of compound $\mathbf{1 4}$ $(1.16 \mathrm{~g}, 4.23 \mathrm{mmol})$ in THF $(15 \mathrm{~mL})$ was added into the reaction mixture at $-78{ }^{\circ} \mathrm{C}$, and slowly warmed to $\mathrm{rt}$ over a period of $3 \mathrm{~h}$. The reaction was quenched with saturated aqueous solution of $\mathrm{NH}_{4} \mathrm{Cl}$ at $0{ }^{\circ} \mathrm{C}$, and the mixture was extracted with EtOAc. The combined organic layers were washed with saturated $\mathrm{NaCl}$ solution, dried over $\mathrm{Na}_{2} \mathrm{SO}_{4}$, and concentrated under reduced pressure. The residue was purified by column chromatography on silica gel (hexanes/EtOAc, 1:1) to afford diol $\mathbf{1 5}(980 \mathrm{mg}, 80 \%)$ as white solid. ${ }^{1} \mathbf{H}$ NMR $\left(\mathbf{5 0 0} \mathbf{M H z}, \mathbf{C D C l}_{3}\right) \boldsymbol{\delta} 6.46(\mathrm{dd}, J=5.5,3.0 \mathrm{~Hz}, 1 \mathrm{H}), 6.15(\mathrm{dd}, J=5.5,3.0 \mathrm{~Hz}, 1 \mathrm{H}), 4.88(\mathrm{~s}, 1 \mathrm{H}), 4.79(\mathrm{~d}, J=$ $2.0 \mathrm{~Hz}, 1 \mathrm{H}), 3.21(\mathrm{dd}, J=11.1,3.1 \mathrm{~Hz}, 1 \mathrm{H}), 3.12(\mathrm{dd}, J=10.8,7.5 \mathrm{~Hz}, 1 \mathrm{H}), 2.94(\mathrm{~s}, 1 \mathrm{H}), 2.73-2.69(\mathrm{~m}, 1 \mathrm{H})$, $2.68(\mathrm{~s}, 1 \mathrm{H}), 2.62(\mathrm{dd}, J=10.9,3.6 \mathrm{~Hz}, 1 \mathrm{H}), 1.80(\mathrm{~s}, 3 \mathrm{H}), 1.73-1.65(\mathrm{~m}, 2 \mathrm{H}), 1.63(\mathrm{t}, J=3.6 \mathrm{~Hz}, 1 \mathrm{H}), 1.59$ $(\mathrm{d}, J=3.9 \mathrm{~Hz}, 1 \mathrm{H}), 1.56(\mathrm{~d}, J=4.2 \mathrm{~Hz}, 1 \mathrm{H}), 1.52(\mathrm{dd}, J=12.4,2.3 \mathrm{~Hz}, 1 \mathrm{H}), 1.49$ (d, $J=8.0 \mathrm{~Hz}, 1 \mathrm{H}), 1.29$ (s,

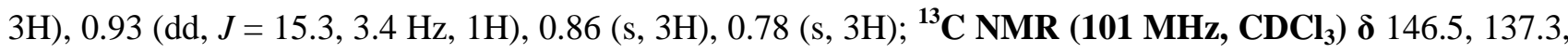
135.2, 113.7, 78.9, 71.4, 58.1, 56.9, 54.8, 51.7, 48.7, 44.6, 43.6, 34.7, 33.6, 27.7, 25.5, 24.4, 18.9; IR (ATR, neat) $v_{\max } 3425,2961,2929,1638,1473,1337,1046,1032,881 \mathrm{~cm}^{-1}$; HRMS (ESI-TOF): calcd. for

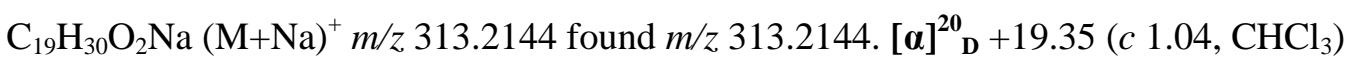

(1S,4S,5R)-5-(3-hydroxy-2,2-dimethylpropyl)-1-methyl-4-(prop-1-en-2-yl)cyclopent-2-en-1-ol<smiles>C=C(C)[C@H]1C=C[C@@](C)(O)C1CC(C)(C)CO</smiles>

Compound15 (390 mg, $1.34 \mathrm{mmol}$ ) was placed in a quartz tube and connected to a pyrolysis apparatus while other end of the receiver connected to a vacuum pump through a cold trap at $-78{ }^{\circ} \mathrm{C}$. Then, the quartz tube was heated to $150-180{ }^{\circ} \mathrm{C}$ at $0.01 \mathrm{~mm} / \mathrm{Hg}$, while the pyrolysis tube $\left(10 \mathrm{~cm}\right.$ long) was kept at $480{ }^{\circ} \mathrm{C}$ until the 
starting material had disappeared. The obtained product from cold trap was further purified by flash chromatography (1:1, n-hexanes/ethyl acetate) to give pure compound 6 (230 mg, 75\%). ${ }^{\mathbf{1}} \mathbf{H}$ NMR (400 MHz, $\left.\mathbf{C D C l}_{3}\right) \boldsymbol{\delta} 5.72(\mathrm{dd}, J=5.7,2.3 \mathrm{~Hz}, 1 \mathrm{H}), 5.68(\mathrm{dd}, J=5.7,1.8 \mathrm{~Hz}, 1 \mathrm{H}), 4.81(\mathrm{~s}, 1 \mathrm{H}), 4.80(\mathrm{~d}, J=1.4 \mathrm{~Hz}, 1 \mathrm{H})$, $3.39(\mathrm{~d}, J=11.3 \mathrm{~Hz}, 1 \mathrm{H}), 3.22(\mathrm{~d}, J=11.3 \mathrm{~Hz}, 1 \mathrm{H}), 3.10(\mathrm{dt}, J=6.8,1.9 \mathrm{~Hz}, 1 \mathrm{H}), 2.60(\mathrm{bs}, 1 \mathrm{H}), 1.98(\mathrm{dd}, J=$ 15.0, 7.1 Hz, 1H), $1.78(\mathrm{td}, J=7.0,3.6 \mathrm{~Hz}, 1 \mathrm{H}), 1.66(\mathrm{~s}, 3 \mathrm{H}), 1.45(\mathrm{~s}, 3 \mathrm{H}), 1.22(\mathrm{dd}, J=15.0,3.6 \mathrm{~Hz}, 1 \mathrm{H})$, $0.93(\mathrm{~s}, 3 \mathrm{H}), 0.86(\mathrm{~s}, 3 \mathrm{H}) ;{ }^{13} \mathbf{C} \mathbf{N M R}\left(\mathbf{1 0 1} \mathbf{~ M H z}, \mathbf{C D C l}_{\mathbf{3}}\right) \boldsymbol{\delta}$ 146.2, 138.0, 136.2, 111.9, 83.2, 70.8, 63.0, 47.2, 37.8, 35.2, 28.4, 26.5, 24.4, 18.9; IR (ATR, neat) $\boldsymbol{v}_{\max } 3301,2956,2869,1645,1473,1092,1047,889 \mathrm{~cm}^{-1}$; HRMS (ESI-TOF): calcd. for $\mathrm{C}_{14} \mathrm{H}_{24} \mathrm{O}_{2} \mathrm{Na}(\mathrm{M}+\mathrm{Na})^{+} m / z 247.1672$ found $m / z 247.1674 ;[\boldsymbol{\alpha}]^{20}{ }_{\mathbf{D}}-122.4(c 0.82$, $\left.\mathrm{CHCl}_{3}\right)$

(4aS,5R,7aR)-3,3,7a-trimethyl-5-(prop-1-en-2-yl)-2,3,4,4a,5,7a-hexahydrocyclopenta[b]pyran-2-ol

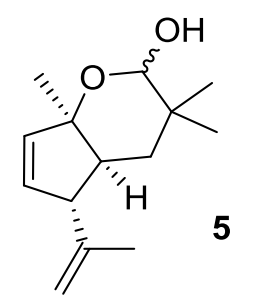

To a stirred solution of compound $6(200 \mathrm{mg}, 0.89 \mathrm{mmol})$ and $\mathrm{Et}_{3} \mathrm{~N}(0.62 \mathrm{~mL}, 4.46 \mathrm{mmol})$ in $\mathrm{CH}_{2} \mathrm{Cl}_{2}(10 \mathrm{~mL})$ was added a solution of $\mathrm{SO}_{3} \cdot \mathrm{Py}(355 \mathrm{mg}, 2.23 \mathrm{mmol})$ in DMSO $(0.5 \mathrm{~mL})$ at $0{ }^{\circ} \mathrm{C}$. After being stirred for $1 \mathrm{~h}$, the mixture was allowed to stir for additional $1 \mathrm{~h}$ at room temperature. The reaction was quenched with water and extracted with EtOAc. The combined extracts were washed with brine, dried over anhydrous Na2SO4 and concentrated in Vacuo. The resultant residue was purified by silica gel flash chromatography (20:1 hexanes:EtOAc) to give $160 \mathrm{mg}$ compound $\mathbf{5}$ in $80 \%$ yield an inseparable diastereomeric mixture. ${ }^{1} \mathbf{H}$ NMR (major isomer) (200 MHz, $\left.\mathbf{C D C l}_{\mathbf{3}}\right) \boldsymbol{\delta} 5.82(\mathrm{dd}, J=5.7,2.3 \mathrm{~Hz}, 1 \mathrm{H}), 5.75(\mathrm{dd}, J=5.7,1.8 \mathrm{~Hz}, 1 \mathrm{H}), 4.76(\mathrm{~s}$, $1 \mathrm{H}), 4.59$ (s, 1H), $4.48(\mathrm{~d}, J=6.7 \mathrm{~Hz}, 1 \mathrm{H}), 2.76(\mathrm{~s}, 1 \mathrm{H}), 2.41(\mathrm{~d}, J=10.3 \mathrm{~Hz}, 1 \mathrm{H}), 1.96(\mathrm{dd}, J=15.0,7.1 \mathrm{~Hz}$, 1H), $1.78(\mathrm{~s}, 3 \mathrm{H}), 1.72-1.68(\mathrm{~m}, 2 \mathrm{H}), 1.33(\mathrm{~s}, 3 \mathrm{H}), 0.86(\mathrm{~s}, 3 \mathrm{H}), 0.51(\mathrm{~s}, 3 \mathrm{H}) ;{ }^{13} \mathbf{C}$ NMR (Major isomer) (101 MHz, $\left.\mathbf{C D C l}_{3}\right) \boldsymbol{\delta} 146.6,136.7,132.2,110.1,98.1,87.2,58.8,43.7,42.1,36.6,33.8,29.3,25.9$, 17.4. IR (ATR, neat) $\mathbf{v m a x} 3358,2972,2931,1648,1480,1443,1287,1157,1083,1061,1005,908 \mathrm{~cm}^{-1} ;[\boldsymbol{\alpha}]^{\mathbf{2 0}}{ }_{\mathbf{D}}^{-}$ $102.3\left(c 0.74, \mathrm{CHCl}_{3}\right)$ 


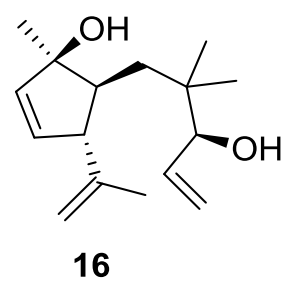

To a solution of lactol $5(150 \mathrm{mg}, 0.67 \mathrm{mmol})$ in anhydrous benzene $(10 \mathrm{~mL})$ was added vinyl magnesium bromide $\left(4.8 \mathrm{~mL}, 0.7 \mathrm{M}\right.$ in THF) dropwise at $0{ }^{\circ} \mathrm{C}$ under nitrogen atmosphere. The mixture was stirred at room temperature for $8 \mathrm{~h}$. The reaction was quenched cautiously with saturated solution of $\mathrm{NH}_{4} \mathrm{Cl}$ at $0{ }^{\circ} \mathrm{C}$ and the aqueous layer was extracted with ethyl acetate $(3 \mathrm{X} 10 \mathrm{~mL})$. The combined organic layers were washed with saturated aqueous $\mathrm{NaCl}$ solution $(10 \mathrm{~mL})$ and concentrated. The obtained crude product was further purified by flash chromatography on silica gel (2:5 hexane:EtOAc) to give $135 \mathrm{mg}$ ( $80 \%$ ) of allylic alcohol 16. ${ }^{1} \mathbf{H}$ NMR (400 MHz, $\mathbf{C D C l}_{3}$ ) $\boldsymbol{\delta} 5.93(\mathrm{ddd}, J=17.0,10.5,6.3 \mathrm{~Hz}, 1 \mathrm{H}), 5.69$ (dd, $\left.J=5.7,2.2 \mathrm{~Hz}, 1 \mathrm{H}\right), 5.65$ $(\mathrm{dd}, J=5.7,1.7 \mathrm{~Hz}, 1 \mathrm{H}), 5.25(\mathrm{dt}, J=17.2,1.6 \mathrm{~Hz}, 1 \mathrm{H}), 5.20-5.17(\mathrm{~m}, 1 \mathrm{H}), 4.77(\mathrm{~s}, 2 \mathrm{H}), 3.93(\mathrm{~d}, J=6.3$ $\mathrm{Hz}, 1 \mathrm{H}), 3.09$ (dt, $J=6.6,1.9 \mathrm{~Hz}, 1 \mathrm{H}), 3.05(\mathrm{bs}, 1 \mathrm{H}), 2.49$ (bs, 1H), 2.15 (dd, $J=15.1,7.7 \mathrm{~Hz}, 1 \mathrm{H}), 1.85$ (td, $J$ = 7.6, 3.2 Hz, 1H), $1.64(\mathrm{t}, J=1.0 \mathrm{~Hz}, 3 \mathrm{H}), 1.47(\mathrm{~s}, 3 \mathrm{H}), 1.19(\mathrm{dd}, J=15.1,3.2 \mathrm{~Hz}, 1 \mathrm{H}), 0.86(\mathrm{~s}, 3 \mathrm{H}), 0.85(\mathrm{~s}$, 3H); ${ }^{13} \mathbf{C}$ NMR (101 MHz, $\left.\mathbf{C D C l}_{3}\right) \boldsymbol{\delta}$ 145.7, 137.8, 137.6, 135.7, 116.2, 111.9, 82.9, 78.5, 63.5, 47.0, 39.7, 37.4, 29.1, 23.9, 23.3, 19.1; IR (ATR, neat) $\boldsymbol{v}_{\max }$ 3338, 2967, 2871, 1645, 1450, 1372, 1083, 996, $891 \mathrm{~cm}^{-1}$; HRMS (ESI-TOF): calcd. for $\mathrm{C}_{16} \mathrm{H}_{26} \mathrm{O}_{2}(\mathrm{M}+\mathrm{Na})^{+} \mathrm{m} / z 273.1831$ found $m / z 273.1832 ;[\boldsymbol{\alpha}]^{\mathbf{2 0}}{ }_{\mathbf{D}}-123.8(c 0.68$, $\left.\mathrm{CHCl}_{3}\right)$

\section{(1R,3aR,6S,8aS)-1,4,7,7-tetramethyl-1,3a,6,7,8,8a-hexahydroazulene-1,6-diol}

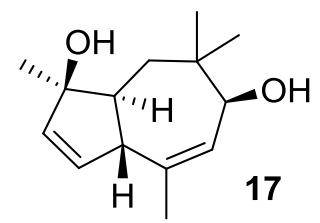

To a solution of diene $\mathbf{1 6}(135 \mathrm{mg}, 0.54 \mathrm{mmol})$ in anhydrous toluene $(50 \mathrm{~mL})$ was added a solution of Grubbs' $2^{\text {nd }}$ generation catalyst $(14 \mathrm{mg}, 0.016 \mathrm{mmol})$ in toluene $(2 \mathrm{~mL})$ at room temperature under nitrogen atmosphere. Then, the reaction mixture was allowed to stir at rt for $15 \mathrm{~min}$, then heated to reflux for $3 \mathrm{~h}$. The reaction allowed to attain room temperature and filtered through a plug of silica gel. The obtained crude material was further purified by flash chromatography to afford compound $\mathbf{1 7}(100 \mathrm{mg}, 80 \%)$ as a pale yellow oil. ${ }^{1} \mathbf{H}$ NMR (400 MHz, $\left.\mathbf{C D C l}_{3}\right) \boldsymbol{\delta} 6.02(\mathrm{dd}, J=5.8,1.6 \mathrm{~Hz}, 1 \mathrm{H}), 5.84(\mathrm{dd}, J=5.8,2.9 \mathrm{~Hz}, 1 \mathrm{H}), 5.60$ $-5.55(\mathrm{~m}, 1 \mathrm{H}), 3.97$ (d, $J=8.8 \mathrm{~Hz}, 1 \mathrm{H}), 3.75(\mathrm{~d}, J=7.4 \mathrm{~Hz}, 1 \mathrm{H}), 2.14(\mathrm{t}, J=13.2 \mathrm{~Hz}, 1 \mathrm{H}), 1.76(\mathrm{dd}, J=1.4$, $1.0 \mathrm{~Hz}, 3 \mathrm{H}), 1.70(\mathrm{ddd}, J=12.6,8.9,2.3 \mathrm{~Hz}, 2 \mathrm{H}), 1.47-1.42(\mathrm{~m}, 1 \mathrm{H}), 1.33(\mathrm{~s}, 3 \mathrm{H}), 1.25-1.22(\mathrm{~m}, 1 \mathrm{H}), 1.09$

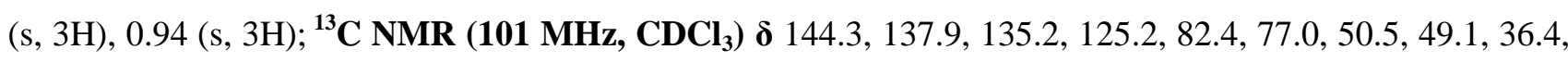


35.7, 30.4, 25.1, 24.9, 22.5; HRMS (ESI-TOF): calcd. for $\mathrm{C}_{14} \mathrm{H}_{22} \mathrm{O}_{2}(\mathrm{M}+\mathrm{Na})+\mathrm{m} / \mathrm{z} 245.1517$ found $\mathrm{m} / \mathrm{z}$ 245.1517; IR (ATR, neat) vmax 3386, 2967, 2929, 1656, 1447, 1375, 1253, 1142, 1078, 1017, $933 \mathrm{~cm}^{-1}$; $[\alpha]^{20}{ }_{D}+52.6\left(c 0.75, \mathrm{CHCl}_{3}\right)$.

(1R,3aR,8aS)-1-hydroxy-1,4,7,7-tetramethyl-3a,7,8,8a-tetrahydroazulen-6(1H)-one

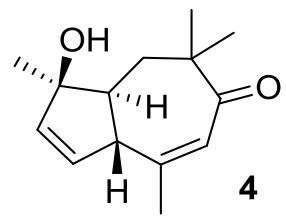

To a slurry of alcohol $17(35 \mathrm{mg}, 0.16 \mathrm{mmol})$ and $\mathrm{NaHCO}_{3}(40 \mathrm{mg}, 0.48 \mathrm{mmol})$ in $\mathrm{CH}_{2} \mathrm{Cl}_{2}(7 \mathrm{~mL})$ at $0{ }^{\circ} \mathrm{C}$ was added Dess-Martin periodinane $(90 \mathrm{mg}, 0.21 \mathrm{mmol})$, and the resultant mixture was stirred for $30 \mathrm{~min}$ at the same temperature. The reaction mixture was diluted with a saturated aqueous $\mathrm{Na}_{2} \mathrm{SO}_{3}$ solution and extracted with $\mathrm{CH}_{2} \mathrm{Cl}_{2}$. The organic layer was washed with brine, dried over $\mathrm{Na}_{2} \mathrm{SO}_{4}$, filtered, and concentrated. The residue was purified by flash column chromatography (silica gel, 30\% EtOAc/hexanes) to give dienone 4 (22 $\mathrm{mg}, 62 \%)$ as a colorless oil.

${ }^{1} \mathbf{H}$ NMR (400 MHz, CDCl $)$ ) $6.14(\mathrm{dd}, J=5.8,1.6 \mathrm{~Hz}, 1 \mathrm{H}), 5.92(\mathrm{dd}, J=5.8,3.1 \mathrm{~Hz}, 1 \mathrm{H}), 5.82(\mathrm{dd}, J=$ 2.9, $1.4 \mathrm{~Hz}, 1 \mathrm{H}), 3.57-3.52(\mathrm{~m}, 1 \mathrm{H}), 2.00(\mathrm{~s}, 3 \mathrm{H}), 1.97-1.78(\mathrm{~m}, 3 \mathrm{H}), 1.38(\mathrm{~s}, 3 \mathrm{H}), 1.25(\mathrm{~s}, 3 \mathrm{H}), 1.23$ (s, 3H); ${ }^{13} \mathbf{C}$ NMR (101 MHz, $\left.\mathbf{C D C l}_{3}\right) \boldsymbol{\delta} 208.1,150.7,138.2,134.1,127.9,82.4,54.0,52.6,46.8,37.6,28.2$, 27.0, 25.5, 23.7; IR (ATR, neat) $v_{\max }$ 2969, 2956, 1656, 1453, 1381, 1271, 1140, 1034, $754 \mathrm{~cm}^{-1}$; HRMS (ESI-TOF): calcd. for $\mathrm{C}_{14} \mathrm{H}_{20} \mathrm{O}_{2}(\mathrm{M}+\mathrm{Na})^{+} \mathrm{m} / z 243.1361$ found $m / z 243.1364 ;[\boldsymbol{\alpha}]^{\mathbf{2 0}}{ }_{\mathbf{D}}-3.65\left(c 0.52, \mathrm{CHCl}_{3}\right)$

(3R,3aS,8aR)-3-hydroxy-3,5,5,8-tetramethyl-2,3,3a,4,5,8a-hexahydroazulen-6(1H)-one

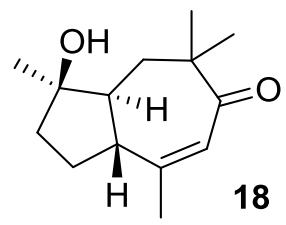

To a solution of diene $4(10 \mathrm{mg}, 45 \mu \mathrm{mol})$ in $\mathrm{MeOH}(3 \mathrm{~mL})$ was added $5 \% \mathrm{Rh}$ on alumina $(2 \mathrm{mg})$ under nitrogen atmosphere at room temperature. The reaction mixture was stirred under hydrogen atmosphere for 4 h. Then, the reaction mixture diluted with EtOAc, filtered through Celite and concentrated in vacuo. The obtained residue was purified by flash chromatography to afford $\alpha, \beta$-unsaturated ester $\mathbf{1 8}(8 \mathrm{mg}, 80 \%)$ as a viscous material. ${ }^{1} \mathbf{H}$ NMR (400 MHz, $\left.\mathbf{C D C l}_{3}\right) \boldsymbol{\delta} 5.80(\mathrm{~s}, 1 \mathrm{H}), 2.70(\mathrm{dd}, \mathrm{J}=12.5,7.5 \mathrm{~Hz}, 1 \mathrm{H}), 2.14-2.04(\mathrm{~m}$, $1 \mathrm{H}), 1.89$ (s, 3H), $1.86-1.80(\mathrm{~m}, 3 \mathrm{H}), 1.66-1.58(\mathrm{~m}, 2 \mathrm{H}), 1.40-1.30(\mathrm{~m}, 2 \mathrm{H}), 1.29$ (s, 3H), $1.16(\mathrm{~s}, 3 \mathrm{H})$, 1.14 (s, 3H); ${ }^{13} \mathbf{C}$ NMR (101 MHz, $\left.\mathbf{C D C l}_{3}\right) \boldsymbol{\delta}$ 208.9, 153.9, 126.6, 80.2, 52.2, 51.5, 47.4, 38.9, 36.8, 29.3, 
27.7, 26.8, 25.8, 24.6; IR (ATR, neat) $\boldsymbol{v}_{\max }$ 3463, 2959, 1623, 1458, 1381, 1108, 1076, $943 \mathrm{~cm}^{-1}$. HRMS (ESI-TOF): calcd. for $\mathrm{C}_{14} \mathrm{H}_{22} \mathrm{O}_{2}(\mathrm{M}+\mathrm{Na})^{+} \mathrm{m} / z 245.1517$ found $\mathrm{m} / \mathrm{z} 245.1516$. [a $]^{\mathbf{2 0}}{ }_{\mathbf{D}}-82.2\left(c 0.51, \mathrm{CHCl}_{3}\right)$

\section{(1R,3aR,6R,8aS)-1,4,7,7-tetramethyl-1,2,3,3a,6,7,8,8a-octahydroazulene-1,6-diol}

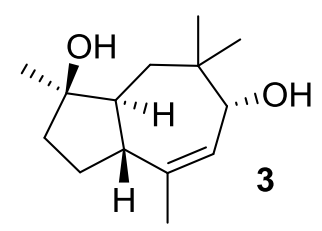

To a solution of DIBAL-H ( $870 \mu \mathrm{L}, 0.95 \mathrm{mmol}, 1.1 \mathrm{M}$ solution in toluene) at $0{ }^{\circ} \mathrm{C}$ was added tert-BuLi (500 $\mu \mathrm{L}, 0.95 \mathrm{mmol}, 1.9 \mathrm{M}$ in pentane) under nitrogen atmosphere and stirred at $0{ }^{\circ} \mathrm{C}$ for $30 \mathrm{mins}$. Then, a solution of enone $(10 \mathrm{mg}, 0.045 \mathrm{mmol})$ in toluene $(1.5 \mathrm{~mL})$ was added into the above reaction mixture of DIBAL$\mathrm{H} / t \mathrm{BuLi}^{1}$ complex in toluene $(0.69 \mathrm{M}, 260 \mu \mathrm{L}, 0.18 \mathrm{mmol})$ at $-78{ }^{\circ} \mathrm{C}$ and stirred for $8 \mathrm{~h}$. The reaction was quenched with $\mathrm{MeOH}$, diluted with $\mathrm{CH}_{2} \mathrm{Cl}_{2}$ and $100 \mu \mathrm{L}$ of $20 \% \mathrm{NaOH}$. The reaction was stirred at room temperature for $8 \mathrm{~h}$. The obtained white precipitate was removed by filtration through a pad of celite and filtrate was concentrated, further purified by flash chromatography to afford diol $\mathbf{3}$ as an amorphous solid (7.3 mg, 71\%). ${ }^{1} \mathbf{H}$ NMR (400 MHz, $\left.\mathbf{C D C l}_{3}\right) \boldsymbol{\delta} 5.28-5.24(\mathrm{~m}, 1 \mathrm{H}), 4.17(\mathrm{~s}, 1 \mathrm{H}), 2.72-2.63(\mathrm{~m}, 1 \mathrm{H}), 1.96-1.87$ $(\mathrm{m}, 1 \mathrm{H}), 1.78(\mathrm{~s}, 3 \mathrm{H}), 1.76-1.69(\mathrm{~m}, 3 \mathrm{H}), 1.63-1.50(\mathrm{~m}, 3 \mathrm{H}), 1.36-1.27(\mathrm{~m}, 2 \mathrm{H}), 1.25(\mathrm{~s}, 3 \mathrm{H}), 1.07$ (s, 3H), 0.87 (s, 3H); ${ }^{13} \mathbf{C}$ NMR (101 MHz, $\mathbf{C D C l}_{3}$ ) $\boldsymbol{\delta}$ 138.9, 130.4, 80.7, 77.4, 48.7, 45.8, 42.5, 41.0, 35.7, 29.2, 26.5, 24.6, 22.1, 19.4; IR (ATR, neat) $\boldsymbol{v}_{\max }$ 2963, 2931, 1652, 1459, 1373, 1081, $1017 \mathrm{~cm}^{-1}$; HRMS (ESITOF): calcd. for $\mathrm{C}_{14} \mathrm{H}_{24} \mathrm{O}_{2}(\mathrm{M}+\mathrm{Na})^{+} \mathrm{m} / z 247.1671$ found $\mathrm{m} / z 247.1674 ;[\boldsymbol{\alpha}]^{\mathbf{2 0}}{ }_{\mathbf{D}}+28.8\left(c 0.37, \mathrm{CHCl}_{3}\right)$.

\section{(+)-omphadiol (1)}

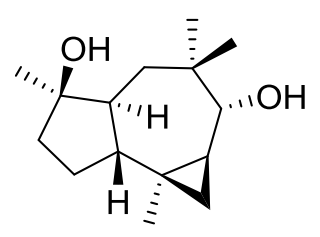

(+)-omphadiol (1)

To a solution of diol 3 (7 mg, $30 \mu \mathrm{mol}, 1.0$ equiv) in $\mathrm{CH}_{2} \mathrm{Cl}_{2}(2 \mathrm{~mL})$ was added $\mathrm{Et}_{2} \mathrm{Zn}(25 \mu \mathrm{L}, 0.31 \mathrm{mmol}, 1 \mathrm{M}$ solution in hexane) drop wise at $-30{ }^{\circ} \mathrm{C}$. After stirring for $10 \mathrm{mins}, \mathrm{CH}_{2} \mathrm{I}_{2}(0.4 \mu \mathrm{L}, \sim 0.5 \mathrm{mmol})$ was added drop wise. The reaction mixture was slowly warmed to $0{ }^{\circ} \mathrm{C}$ and stirred at this temperature for $2 \mathrm{~h}$. The reaction was then quenched with $\mathrm{H}_{2} \mathrm{O}$, diluted with EtOAc, washed with $1 \mathrm{M} \mathrm{NaOH}$ followed by brine, dried over sodium sulfate, concentrated, and purified by flash column chromatography $\left(\mathrm{SiO}_{2}\right.$, eluting with 20 to $50 \%$ EtOAc/hexanes) to afford (+)-omphadiol 1 as an amorphous solid (5.8 $\mathrm{mg}, 78 \%$ ). IR (ATR, neat) $\boldsymbol{v}_{\max } 3347$, 2948, 2929, 1467, $1373 \mathrm{~cm}^{-1}$; $[\alpha]_{\mathrm{D}}{ }^{23}+20.1$ (c 0.36, EtOH); HRMS (ESI-TOF)): calcd. for $\mathrm{C}_{15} \mathrm{H}_{27} \mathrm{O}_{2}(\mathrm{M}+1)^{+}$ $m / z$ 239.1933 found $m / z$ 239.1933; For ${ }^{1} \mathrm{H}$ and ${ }^{13} \mathrm{C}$ NMR, see comparison Tables 1 and 2 below; 
Comparison Table-1: $\left({ }^{1} \mathrm{H}\right.$ NMR characterization data)

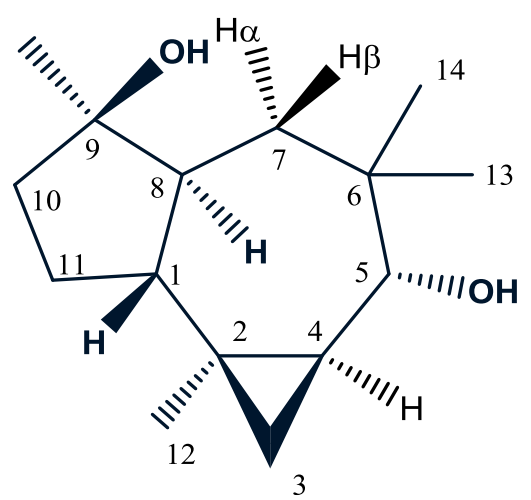

\begin{tabular}{|c|c|c|c|c|}
\hline $\begin{array}{l}{ }^{1} \mathrm{H} \\
\text { NMR }\end{array}$ & $\begin{array}{l}\text { Natural omphadiol }^{2} \\
(600 \mathrm{MHz} \\
\left.\left(\mathrm{CD}_{3}\right)_{2} \mathrm{CO}\right)\end{array}$ & $\begin{array}{l}\text { Synthetic omphadiol }^{3} \\
(500 \mathrm{MHz} \\
\left.\left(\mathrm{CD}_{3}\right)_{2} \mathrm{CO}\right)\end{array}$ & $\begin{array}{l}\text { Synthetic omphadiol }{ }^{4} \\
\left(400 \mathrm{MHz},\left(\mathrm{CD}_{3}\right)_{2} \mathrm{CO}\right)\end{array}$ & $\begin{array}{l}\text { Our synthetic } \\
\text { omphadiol } \\
\left(400 \mathrm{MHz},\left(\mathrm{CD}_{3}\right)_{2} \mathrm{CO}\right)\end{array}$ \\
\hline 1 & 1.45 (overlap) & $1.45(\mathrm{~m})$ & $1.46(\mathrm{dd}, J=10.5,3.6)$ & $1.48-1.44(\mathrm{~m})$ \\
\hline $3 \alpha$ & $0.34(\mathrm{t}, J=4.4)$ & $0.34(\mathrm{t}, J=4.4)$ & $0.34(\mathrm{t}, J=4.3)$ & $0.36(\mathrm{t}, \mathrm{J}=4.4 \mathrm{~Hz})$ \\
\hline $3 \boldsymbol{\beta}$ & $\begin{array}{l}0.62 \\
4.0)\end{array} \quad(\mathrm{dd}, J=8.2$, & $0.62(\mathrm{dd}, J=8.5,4.0)$ & $0.61(\mathrm{dd}, J=8.1,3.9)$ & $\begin{array}{l}0.63(\mathrm{dd}, \mathrm{J}=8.2,3.9 \\
\mathrm{Hz})\end{array}$ \\
\hline 4 & $0.51-0.55(\mathrm{~m})$ & $0.51-0.55(\mathrm{~m})$ & $0.51-0.56(\mathrm{~m})$ & $0.57-0.53(\mathrm{~m})$ \\
\hline $5 \alpha$ & $\begin{array}{l}3.05(\mathrm{dd}, J=9.0,4.2 \\
\text { ) }\end{array}$ & $\begin{array}{l}3.05(\mathrm{dd}, J=8.5,4.5 \\
)\end{array}$ & $3.03(\mathrm{dd}, J=8.5,4.4)$ & $\begin{array}{l}3.06(\mathrm{dd}, \mathrm{J}=8.5,4.5 \\
\mathrm{Hz})\end{array}$ \\
\hline $7 \alpha$ & $1.25-1.29(\mathrm{~m})$ & $1.23-1.29(\mathrm{~m})$ & $1.22-1.30(\mathrm{~m})$ & $1.30-1.24(\mathrm{~m})$ \\
\hline $7 \beta$ & 1.42 (overlap) & $1.42(\mathrm{~m})$ & $1.42(\mathrm{t}, J=3.5)$ & $1.44-1.41(\mathrm{~m})$ \\
\hline 8 & 1.59 (overlap) & $1.59(\mathrm{~m})$ & $1.51-1.60(\mathrm{~m})$ & $1.62-1.51(\mathrm{~m})$ \\
\hline $10 \alpha$ & 1.58 (overlap) & $1.58(\mathrm{~m})$ & $1.51-1.60(\mathrm{~m})$ & $1.61-1.55(\mathrm{~m})$ \\
\hline $10 \beta$ & 1.65 (overlap) & $1.65(\mathrm{~m})$ & $1.60-1.66(\mathrm{~m})$ & $1.60-1.66$ \\
\hline $11 \alpha$ & 1.64 (overlap) & $1.64(\mathrm{~m})$ & $1.60-1.66(\mathrm{~m})$ & $1.62-1.68$ \\
\hline $11 \beta$ & $1.77-1.80(\mathrm{~m})$ & $1.75-1.82(\mathrm{~m})$ & $\begin{array}{l}1.79(\mathrm{ddd}, J=18.1, \\
10.5,5.0)\end{array}$ & $1.83-1.76(\mathrm{~m}, 2 \mathrm{H})$ \\
\hline 12 & $0.98(\mathrm{~s}, 3 \mathrm{H})$ & $0.98(\mathrm{~s}, 3 \mathrm{H})$ & $0.98(\mathrm{~s}, 3 \mathrm{H})$ & $0.99(\mathrm{~s}, 3 \mathrm{H})$ \\
\hline 13 & $0.97(\mathrm{~s}, 3 \mathrm{H})$ & $0.97(\mathrm{~s}, 3 \mathrm{H})$ & $0.97(\mathrm{~s}, 3 \mathrm{H})$ & $0.97(\mathrm{~s}, 3 \mathrm{H})$ \\
\hline 14 & $0.94(\mathrm{~s}, 3 \mathrm{H})$ & $0.94(\mathrm{~s}, 3 \mathrm{H})$ & $0.94(\mathrm{~s}, 3 \mathrm{H})$ & $0.95(\mathrm{~s}, 3 \mathrm{H})$ \\
\hline 15 & $1.21(\mathrm{~s}, 3 \mathrm{H})$ & $1.21(\mathrm{~s}, 3 \mathrm{H})$ & $1.21(\mathrm{~s}, 3 \mathrm{H})$ & $1.23(\mathrm{~s}, 6 \mathrm{H})$ \\
\hline $5(\mathrm{OH})$ & $3.40(\mathrm{~d}, J=4.2)$ & $3.41(\mathrm{~d}, J=4.5)$ & $3.40(\mathrm{~d}, J=4.5)$ & $3.40(\mathrm{~d}, \mathrm{~J}=4.4 \mathrm{~Hz})$ \\
\hline $9(\mathrm{OH})$ & $2.90(\mathrm{~s})$ & $2.90(\mathrm{~s})$ & $2.89(\mathrm{~s})$ & $2.90(\mathrm{~s}, 1 \mathrm{H})$ \\
\hline
\end{tabular}


Comparison Table-2: $\left({ }^{13} \mathrm{C}\right.$ NMR characterization data)

\begin{tabular}{|c|c|c|c|c|c|}
\hline $13 \mathrm{C}$ & $\begin{array}{l}\text { Natural } \\
\text { omphadiol }^{2} \\
(150 \mathrm{MHz}, \\
\left.\left(\mathrm{CD}_{3}\right)_{2} \mathrm{CO}\right)\end{array}$ & $\begin{array}{l}\text { Synthetic } \\
\text { omphadiol }^{3} \\
(125 \mathrm{MHz}, \\
\left.\left(\mathrm{CD}_{3}\right)_{2} \mathrm{CO}\right)\end{array}$ & $\begin{array}{l}\text { Synthetic } \\
\text { omphadiol }^{4} \\
(100 \mathrm{MHz}, \\
\left.\left(\mathrm{CD}_{3}\right)_{2} \mathrm{CO}\right)\end{array}$ & $\begin{array}{l}\text { Our synthetic } \\
\text { omphadiol }_{(100 \mathrm{MHz},} \\
\left.\left(\mathrm{CD}_{3}\right)_{2} \mathrm{CO}\right)\end{array}$ & $\Delta \delta$ \\
\hline 1 & 49.2 & 49.2 & 50.2 & $\mathbf{4 9 . 2}$ & $\mathbf{0}$ \\
\hline $\mathbf{2}$ & 18.8 & 18.8 & 19.8 & $\mathbf{1 8 . 8}$ & $\mathbf{0}$ \\
\hline $\mathbf{3}$ & 22.5 & 22.5 & 23.5 & $\mathbf{2 2 . 5}$ & $\mathbf{0}$ \\
\hline $\mathbf{4}$ & 30.3 & 30.3 & 31.3 & $\mathbf{3 0 . 3}$ & $\mathbf{0}$ \\
\hline $\mathbf{5}$ & 79.8 & 79.8 & 80.8 & $\mathbf{3 7 . 8}$ & $\mathbf{0}$ \\
\hline $\mathbf{6}$ & 37.8 & 37.8 & 38.8 & $\mathbf{4 2 . 4}$ & $\mathbf{0}$ \\
\hline $\mathbf{7}$ & 42.4 & 42.4 & 43.4 & $\mathbf{4 8 . 3}$ & $\mathbf{0}$ \\
\hline $\mathbf{8}$ & 48.3 & 48.3 & 49.3 & $\mathbf{4 1 . 4}$ & $\mathbf{0}$ \\
\hline $\mathbf{9}$ & 79.4 & 79.4 & 80.4 & $\mathbf{2 3 . 0}$ & $\mathbf{0}$ \\
\hline $\mathbf{1 0}$ & 41.4 & 41.4 & 42.4 & $\mathbf{1 9 . 1}$ & $\mathbf{0 . 1}$ \\
\hline $\mathbf{1 1}$ & 23.0 & 23.0 & 24.0 & $\mathbf{2 8 . 9}$ & $\mathbf{1 . 3}$ \\
\hline $\mathbf{1 2}$ & 19.0 & 19.1 & 20.0 & $\mathbf{1 8 . 9}$ & $\mathbf{0}$ \\
\hline $\mathbf{1 3}$ & 30.0 & 28.8 & 29.8 & $\mathbf{2 5 . 0}$ & $\mathbf{0}$ \\
\hline $\mathbf{1 4}$ & 18.9 & 18.9 & 19.8 & 26.0 & \\
\hline $\mathbf{1 5}$ & 25.0 & 25.0 & & &
\end{tabular}

\section{References:}

1. Bian, J.; Van Wingerden, M.; Ready, J. M., J. Am. Chem. Soc. 2006, 128, 7428.

2. Zheng, Y.-B.; Lu, C.-H.; Zheng, Z.-H.; Lin, X.-H.; Su, W.-J.; Shen, Y.-M. Helv. Chim. Acta 2008, 91, 2174.

3. Liu, G.; Romo, D. Angew. Chem. Int. Ed. 2011, 50, 7537.

4. Zhou, L.; Yao, Y.; Xu, W.; Liang, G. J. Org. Chem. 2014, 79, 5345. 


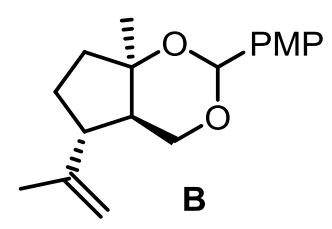

To a solution of diol $23(1.79 \mathrm{~g}, 10.5 \mathrm{mmol})$ in $\mathrm{CH}_{2} \mathrm{Cl}_{2}(10.5 \mathrm{~mL})$ was added p-anisaldehyde dimethylacetal $(1.97 \mathrm{~mL}, 1.10 \mathrm{eq}, 11.6 \mathrm{mmol})$ and PPTS $(265 \mathrm{mg}, 0.10 \mathrm{eq}, 1.05 \mathrm{mmol})$ at $0{ }^{\circ} \mathrm{C}$ allowed to react at room temperature for $4 \mathrm{~h}$. The mixture was diluted with $\mathrm{CH}_{2} \mathrm{Cl}_{2}$, and then washed with sat.aq. $\mathrm{NaHCO}_{3}$, dried $\left(\mathrm{MgSO}_{4}\right)$ and concentrated. The obtained residue was purified by flash chromatography (PE:MTBE, 9:1) to afford acetal $\mathbf{B}$ as a pale yellow oil (2.53 g, 83\%). ${ }^{1} \mathbf{H}$ NMR (400 $\left.\mathbf{M H z}, \mathbf{C D C l}_{\mathbf{3}}\right) \boldsymbol{\delta} 7.48-7.37$ (m, 2H), $6.94-$ $6.85(\mathrm{~m}, 2 \mathrm{H}), 5.62(\mathrm{~s}, 1 \mathrm{H}), 4.78(\mathrm{~s}, 1 \mathrm{H}), 4.77-4.72(\mathrm{~m}, 1 \mathrm{H}), 4.09(\mathrm{dd}, J=11.8,2.9 \mathrm{~Hz}, 1 \mathrm{H}), 3.97(\mathrm{~d}, J=11.5$ $\mathrm{Hz}, 1 \mathrm{H}), 3.80(\mathrm{~s}, 3 \mathrm{H}), 3.28(\mathrm{dd}, J=17.5,9.8 \mathrm{~Hz}, 1 \mathrm{H}), 2.13(\mathrm{ddd}, J=16.9,12.7,8.9 \mathrm{~Hz}, 1 \mathrm{H}), 1.98(\mathrm{ddd}, J=$ 13.3, 8.7, 4.4 Hz, 1H), $1.82-1.73(\mathrm{~m}, 1 \mathrm{H}), 1.72(\mathrm{~s}, 3 \mathrm{H}), 1.64-1.53(\mathrm{~m}, 1 \mathrm{H}), 1.52(\mathrm{~s}, 3 \mathrm{H}), 1.45(\mathrm{dd}, J=10.4$, $1.6 \mathrm{~Hz}, 1 \mathrm{H}) ;{ }^{13} \mathbf{C} \mathbf{N M R}\left(\mathbf{1 0 1} \mathbf{M H z}, \mathbf{C D C l}_{3}\right) \boldsymbol{\delta} 159.9,146.9,131.9,127.5,113.7,110.5,95.6,82.2,63.9,55.3$, 47.3, 45.9, 41.1, 27.9, 20.8, 19.4; HRMS (ESI-TOF): calcd. for $\mathrm{C}_{18} \mathrm{H}_{25} \mathrm{O}_{3}(\mathrm{M}+1)^{+} \mathrm{m} / \mathrm{z} 289.1804$ found $\mathrm{m} / \mathrm{z}$

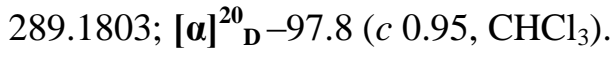

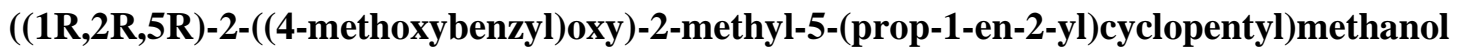

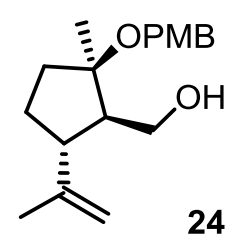

To a solution of above acetal $\mathbf{B}(1.23 \mathrm{~g}, 4.28 \mathrm{mmol})$ in $\mathrm{CH}_{2} \mathrm{Cl}_{2}(20 \mathrm{~mL})$ was added DIBAL-H (1 M solution in $\mathrm{CH}_{2} \mathrm{Cl}_{2}, 2.50 \mathrm{eq}, 10.69 \mathrm{~mL}$ ) dropwise at $-78{ }^{\circ} \mathrm{C}$. The resulting mixture was stirred for $1 \mathrm{~h}$ at $-78{ }^{\circ} \mathrm{C}$ and then allowed to reach $0{ }^{\circ} \mathrm{C}$. Then the mixture was diluted with MTBE and quenched with sat. aq. Rochelle salt solution carefully. The reaction mixture was stirred for $30 \mathrm{~min}$ and the separated organic layer was washed with brine, dried $\left(\mathrm{MgSO}_{4}\right)$ and evaporated. The crude product was purified by column chromatography (PE:MTBE, 85:15) to yield compound $\mathbf{2 4}$ as a colorless oil $(2.12 \mathrm{~g}, 82 \%) .{ }^{1} \mathbf{H} \mathbf{N M R}\left(\mathbf{4 0 0} \mathbf{M H z}, \mathbf{C D C l}_{3}\right) \boldsymbol{\delta}$ $7.21(\mathrm{~d}, J=8.7 \mathrm{~Hz}, 2 \mathrm{H}), 6.87(\mathrm{~d}, J=8.7 \mathrm{~Hz}, 2 \mathrm{H}), 4.84-4.78(\mathrm{~m}, 1 \mathrm{H}), 4.77-4.72(\mathrm{~m}, 1 \mathrm{H}), 4.41(\mathrm{~d}, J=10.7$ $\mathrm{Hz}, 1 \mathrm{H}), 4.34(\mathrm{~d}, J=10.7 \mathrm{~Hz}, 1 \mathrm{H}), 3.89(\mathrm{dt}, J=11.6,2.9 \mathrm{~Hz}, 1 \mathrm{H}), 3.80$ (s, 3H), $3.63(\mathrm{ddd}, J=11.6,9.0,5.0$ $\mathrm{Hz}, 1 \mathrm{H}), 3.18(\mathrm{dd}, J=9.0,3.1 \mathrm{~Hz}, 1 \mathrm{H}), 2.97(\mathrm{dd}, J=19.0,8.6 \mathrm{~Hz}, 1 \mathrm{H}), 2.14-2.03(\mathrm{~m}, 1 \mathrm{H}), 1.98-1.87(\mathrm{~m}$, 1H), $1.72(\mathrm{~s}, 3 \mathrm{H}), 1.67-1.60(\mathrm{~m}, 2 \mathrm{H}), 1.59-1.51(\mathrm{~m}, 1 \mathrm{H}), 1.44(\mathrm{~s}, 3 \mathrm{H}) ;{ }^{13} \mathbf{C} \mathbf{N M R}\left(\mathbf{1 0 1} \mathbf{M H z}, \mathbf{C D C l}_{3}\right) \boldsymbol{\delta}$ $158.97,146.99,131.02,128.62,113.86,110.55,87.38,64.03,60.47,55.25,54.64,46.06,35.67,28.14,23.20$,

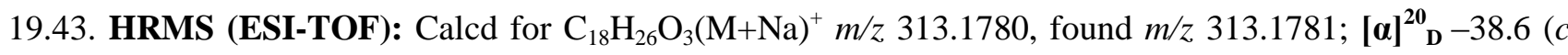
$\left.1.41, \mathrm{CHCl}_{3}\right)$. 
((1R,2R,5R)-2-((4-methoxybenzyl)oxy)-2-methyl-5-(prop-1-en-2-yl)cyclopentyl)methyl 4methylbenzenesulfonate

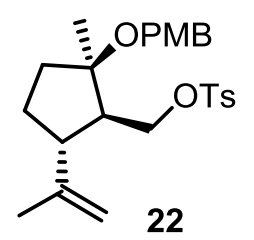

To a solution of alcohol $24(1.45 \mathrm{~g}, 5.00 \mathrm{mmol})$ in pyridine $(5 \mathrm{~mL})$ was added 4-toluenesulfonyl chloride $(1.00 \mathrm{~g}, 1.05 \mathrm{eq}, 5.25 \mathrm{mmol})$ and the mixture was stirred for $18 \mathrm{~h}$ at room temperature. Then reaction was diluted with water and extracted with $\operatorname{MTBE}(3 \times 10 \mathrm{~mL})$. The combined organic layers were washed with $1 \mathrm{M}$ aq. $\mathrm{HCl}$ solution $(5 \mathrm{~mL})$, brine and dried $\left(\mathrm{MgSO}_{4}\right)$. After removal of the solvent, the obtained crude product was purified by column chromatography (PE:MTBE, 9:1) to afford compound 22 as colorless crystals (1.92 g, 86\%). ${ }^{1} \mathbf{H}$ NMR (400 MHz, $\left.\mathbf{C D C l}_{3}\right) \boldsymbol{\delta} 7.77(\mathrm{~d}, J=8.3 \mathrm{~Hz}, 2 \mathrm{H}), 7.28(\mathrm{~d}, J=7.9 \mathrm{~Hz}, 2 \mathrm{H}), 7.13(\mathrm{~d}, J=8.7 \mathrm{~Hz}$, 2H), $6.86(\mathrm{~d}, J=8.7 \mathrm{~Hz}, 2 \mathrm{H}), 4.72-4.67(\mathrm{~m}, 1 \mathrm{H}), 4.66(\mathrm{~s}, 1 \mathrm{H}), 4.40(\mathrm{dd}, J=9.7,8.6 \mathrm{~Hz}, 1 \mathrm{H}), 4.29(\mathrm{~d}, J=$ $11.1 \mathrm{~Hz}, 1 \mathrm{H}), 4.26(\mathrm{~d}, J=11.1 \mathrm{~Hz}, 1 \mathrm{H}), 3.99(\mathrm{dd}, J=9.8,4.2 \mathrm{~Hz}, 1 \mathrm{H}), 3.84(\mathrm{~s}, 3 \mathrm{H}), 2.56-2.46(\mathrm{~m}, 1 \mathrm{H}), 2.43$ (s, 3H), $2.14-1.97(\mathrm{~m}, 2 \mathrm{H}), 1.91-1.80(\mathrm{~m}, 1 \mathrm{H}), 1.66(\mathrm{~s}, 3 \mathrm{H}), 1.56-1.46(\mathrm{~m}, 2 \mathrm{H}), 1.41(\mathrm{~s}, 3 \mathrm{H}) ;{ }^{13} \mathbf{C} \mathbf{N M R}$ $\left(\mathbf{1 0 1} \mathbf{~ M H z}, \mathbf{C D C l}_{3}\right) \boldsymbol{\delta} 158.7,146.1,144.3,133.2,131.7,129.6,128.2,127.3,113.6,111.1,83.5,69.5,63.3$, 55.3, 52.3, 48.5, 35.0, 27.8, 22.5, 21.6, 18.8; HRMS (ESI-TOF): $\mathrm{m} / \mathrm{z}$ Calcd for $\mathrm{C}_{25} \mathrm{H}_{32} \mathrm{O}_{5} \mathrm{~S}(\mathrm{M}+\mathrm{Na})^{+} \mathrm{m} / \mathrm{z}$ 467.1868 , found $\mathrm{m} / \mathrm{z}, 467.1854 ;[\mathbf{\alpha}]^{20}{ }_{\mathbf{D}}-56.0\left(c 0.52, \mathrm{CHCl}_{3}\right)$.

\section{3-((1S,2R,5R)-2-((4-methoxybenzyl)oxy)-2-methyl-5-(prop-1-en-2-yl)cyclopentyl)-2,2-}

\section{dimethylpropanenitrile}

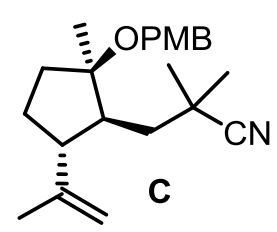

To a solution of diisopropylamine $(350 \mu \mathrm{L}, 2.50 \mathrm{eq}, 2.50 \mathrm{mmol})$ in THF $(4 \mathrm{~mL})$ was added $\mathrm{n}-\mathrm{BuLi}(1.00 \mathrm{~mL}$, $2.50 \mathrm{eq}, 2.50 \mathrm{mmol}, 2.5 \mathrm{M}$ solution in hexane) at $0{ }^{\circ} \mathrm{C}$ and stirred for $30 \mathrm{~min}$. Then, isobutyronitrile $(224 \mu \mathrm{L}$, $2.50 \mathrm{eq}, 2.50 \mathrm{mmol}$ ) was introduced into the reaction mixture and stirring was continued for $30 \mathrm{~min}$. Later, a solution of tosylate $22(222 \mathrm{mg}, 0.500 \mathrm{mmol})$ in THF $(2 \mathrm{~mL})$ was added drop wise at $0{ }^{\circ} \mathrm{C}$ and the mixture was allowed to reach room temperature for overnight. The reaction was quenched with water, the organic layer was separated and the aqueous layer was extracted with ethyl acetate. The combined organic layers were washed with and dried $\left(\mathrm{MgSO}_{4}\right)$. After concentration, the obtained crude product was purified by column chromatography to afford the nitrile $\mathbf{C}$ as colorless oil (147 mg, 86\%). ${ }^{1} \mathbf{H}$ NMR (400 $\left.\mathbf{M H z}, \mathbf{C D C l}_{\mathbf{3}}\right) \boldsymbol{\delta} 7.23$ (d, $J=8.7 \mathrm{~Hz}, 2 \mathrm{H}), 6.88(\mathrm{~d}, J=8.7 \mathrm{~Hz}, 2 \mathrm{H}), 4.78(\mathrm{~d}, J=2.0 \mathrm{~Hz}, 1 \mathrm{H}), 4.76-4.71(\mathrm{~m}, 1 \mathrm{H}), 4.38(\mathrm{~d}, J=11.0 \mathrm{~Hz}$, $1 \mathrm{H}), 4.31(\mathrm{~d}, J=11.0 \mathrm{~Hz}, 1 \mathrm{H}), 3.81(\mathrm{~s}, 3 \mathrm{H}), 2.59(\mathrm{td}, J=9.6,5.8 \mathrm{~Hz}, 1 \mathrm{H}), 2.21(\mathrm{dd}, J=15.1,4.2 \mathrm{~Hz}, 1 \mathrm{H})$, $2.13-2.03(\mathrm{~m}, 1 \mathrm{H}), 1.94-1.83(\mathrm{~m}, 1 \mathrm{H}), 1.74(\mathrm{~s}, 3 \mathrm{H}), 1.73-1.69(\mathrm{~m}, 1 \mathrm{H}), 1.57-1.50(\mathrm{~m}, 2 \mathrm{H}), 1.42(\mathrm{~s}$, 
3H), $1.36(\mathrm{~m}, 1 \mathrm{H}), 1.33(\mathrm{~s}, 3 \mathrm{H}), 1.26(\mathrm{~s}, 3 \mathrm{H}) ;{ }^{13} \mathbf{C} \mathbf{~ N M R}\left(\mathbf{1 0 1} \mathbf{~ M H z}, \mathbf{C D C l}_{\mathbf{3}}\right) \boldsymbol{\delta}$ 158.7, 147.2, 132.0, 128.2, 125.9, 113.6, 111.6, 84.8, 63.4, 55.3, 54.2, 50.2, 39.6, 35.1, 32.2, 28.5, 28.0, 27.2, 22.2, 19.0; HRMS (ESITOF): Calcd for $\mathrm{C}_{22} \mathrm{H}_{31} \mathrm{NO}_{2}(\mathrm{M}+\mathrm{Na})^{+} \mathrm{m} / z$ 364.2252, found $364.2251 ;[\boldsymbol{\alpha}]^{\mathbf{2 0}}{ }_{\mathbf{D}}-58.3\left(c 0.24, \mathrm{CHCl}_{3}\right)$.

3-((1S,2R,5R)-2-((4-methoxybenzyl)oxy)-2-methyl-5-(prop-1-en-2-yl)cyclopentyl)-2,2-dimethylpropanal

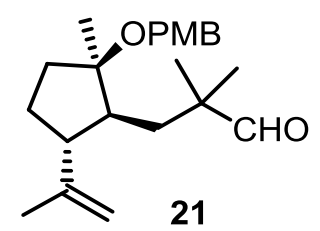

To a solution of above compound $(1.00 \mathrm{~g}, 2.93 \mathrm{mmol})$ in $\mathrm{CH}_{2} \mathrm{Cl}_{2}(29 \mathrm{~mL})$ was added DIBAl-H $(8.79 \mathrm{~mL}, 3.00$ eq, $8,79 \mathrm{mmol}, 1 \mathrm{M}$ solution in $\mathrm{CH}_{2} \mathrm{Cl}_{2}$ ) dropwise at $-78{ }^{\circ} \mathrm{C}$. The mixture was stirred for $1 \mathrm{~h}$ and then allowed to reach $0{ }^{\circ} \mathrm{C}$. The reaction was quenched with Sat. aq. Rochelle salt solution followed by addition of MTBE $(20 \mathrm{~mL})$ and the mixture was stirred for $30 \mathrm{~min}$. The organic layer was separated, washed with brine and dried over $\mathrm{MgSO}_{4}$. Column chromatography (PE/MTBE, 95:5) of the crude product afforded aldehyde 21 as colorless oil (1.00 g, 99\%). ${ }^{1} \mathbf{H}$ NMR (400 MHz, $\left.\mathbf{C D C l}_{3}\right) \boldsymbol{\delta} 9.31(\mathrm{~s}, 1 \mathrm{H}), 7.25(\mathrm{~d}, J=8.8 \mathrm{~Hz}, 2 \mathrm{H}), 6.88(\mathrm{~d}, J=$ $8.7 \mathrm{~Hz}, 2 \mathrm{H}), 4.82-4.74(\mathrm{~m}, 1 \mathrm{H}), 4.72(\mathrm{dd}, J=2.4,1.4 \mathrm{~Hz}, 1 \mathrm{H}), 4.34(\mathrm{~d}, J=11.0 \mathrm{~Hz}, 1 \mathrm{H}), 4.28(\mathrm{~d}, J=11.0$ $\mathrm{Hz}, 1 \mathrm{H}), 3.81(\mathrm{~s}, 3 \mathrm{H}), 2.49$ (dd, $J=17.4,9.2 \mathrm{~Hz}, 1 \mathrm{H}), 2.07$ (dd, $J=15.1,5.5 \mathrm{~Hz}, 1 \mathrm{H}), 2.05-1.96(\mathrm{~m}, 1 \mathrm{H})$, $1.81(\mathrm{ddt}, J=15.0,12.6,6.0 \mathrm{~Hz}, 1 \mathrm{H}), 1.63(\mathrm{~d}, J=0.5 \mathrm{~Hz}, 3 \mathrm{H}), 1.59-1.42(\mathrm{~m}, 3 \mathrm{H}), 1.37$ (dd, $J=15.1,4.3$ Hz, 1H), 1.27 (s, 3H), 1.00 (s, 3H), 0.98 (s, 3H); ${ }^{13} \mathbf{C}$ NMR (101 MHz, $\mathbf{C D C l}_{\mathbf{3}}$ ) $\boldsymbol{\delta}$ 206.7, 158.7, 147.0, 132.0, 128.5, 113.6, 111.8, 84.3, 63.5, 55.3, 55.0, 49.1, 45.5, 36.8, 36.1, 28.0, 23.0, 22.8, 21.8, 18.5; HRMS (ESITOF): Calcd for $\mathrm{C}_{22} \mathrm{H}_{32} \mathrm{O}_{3}(\mathrm{M}+\mathrm{Na})^{+} \mathrm{m} / z 367.2249$, found $m / z 367.2249 ;[\boldsymbol{\alpha}]^{\mathbf{2 0}}{ }_{\mathbf{D}}-61.2\left(c 0.50, \mathrm{CHCl}_{3}\right)$.

4-((1S,2R,5R)-2-((4-methoxybenzyl)oxy)-2-methyl-5-(prop-1-en-2-yl)cyclopentyl)-3,3-dimethylbutanal

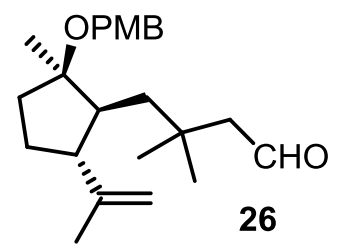

To a solution of (methoxymethyl)diphenylphosphine oxide (985 mg, $2.00 \mathrm{eq}, 4.00 \mathrm{mmol})$ in THF (16 mL) was added KHMDS solution $\left(4.00 \mathrm{~mL}, 2.00 \mathrm{eq}, 4.00 \mathrm{mmol}, 1 \mathrm{M}\right.$ solution in THF) dropwise ${ }^{1}$. The resultant yellow suspension was stirred for $30 \mathrm{~min}$ and then aldehyde 21 (689 mg, $2.00 \mathrm{mmol})$ in THF (4 mL) was added. The reaction mixture was stirred for $16 \mathrm{~h}$ and then quenched with sat. aq. $\mathrm{NH}_{4} \mathrm{Cl}$ solution $(5 \mathrm{~mL})$. The organic layer was separated, washed with brine, dried $\left(\mathrm{MgSO}_{4}\right)$ and evaporated. The unreacted Wittig reagent was removed by short column filtration to afford crude enol ether $\mathbf{2 5}$ which was dissolved in THF (10 mL) and allowed to react with conc. $\mathrm{HCl}$ solution $(0.5 \mathrm{~mL})$. After complete consumption of starting material $(2 \mathrm{~h})$ by TLC, the reaction was basified with sat. aq. $\mathrm{NaHCO}_{3}$ solution cautiously. The separated organic layer was washed with brine, dried $\left(\mathrm{MgSO}_{4}\right)$ and concentrated. Purification by column chromatography (PE:MTBE, 95:5) afforded aldehyde 26 as colorless oil (612 mg, 85\%). ${ }^{1} \mathbf{H}$ NMR (400 MHz, $\left.\mathbf{C D C l}_{3}\right) \boldsymbol{\delta} 9.82(\mathrm{t}, J=3.1 \mathrm{~Hz}$, 
1H), $7.23(\mathrm{~d}, J=8.6 \mathrm{~Hz}, 2 \mathrm{H}), 6.88(\mathrm{~d}, J=8.7 \mathrm{~Hz}, 2 \mathrm{H}), 4.76(\mathrm{~s}, 1 \mathrm{H}), 4.71(\mathrm{~s}, 1 \mathrm{H}), 4.36(\mathrm{~d}, J=11.2 \mathrm{~Hz}, 1 \mathrm{H})$, $4.31(\mathrm{~d}, J=11.3 \mathrm{~Hz}, 1 \mathrm{H}), 3.81(\mathrm{~s}, 3 \mathrm{H}), 2.57(\mathrm{td}, J=9.5,5.4 \mathrm{~Hz}, 1 \mathrm{H}), 2.28-2.13(\mathrm{~m}, 2 \mathrm{H}), 2.07(\mathrm{dd}, J=10.2$, $7.8 \mathrm{~Hz}, 1 \mathrm{H}), 1.99(\mathrm{dd}, J=15.1,3.5 \mathrm{~Hz}, 1 \mathrm{H}), 1.93-1.82(\mathrm{~m}, 1 \mathrm{H}), 1.71(\mathrm{~s}, 3 \mathrm{H}), 1.63-1.56(\mathrm{~m}, 1 \mathrm{H}), 1.52-$ $1.44(\mathrm{~m}, 2 \mathrm{H}), 1.36-1.23(\mathrm{~m}, 1 \mathrm{H}), 1.25$ (s, 3H), 1.00 (s, 3H), $0.98(\mathrm{~s}, 3 \mathrm{H}) ;{ }^{13} \mathbf{C} \mathbf{~ N M R}\left(\mathbf{1 0 1} \mathbf{M H z}, \mathbf{C D C l}_{3}\right) \boldsymbol{\delta}$ 204.3, 158.7, 147.8, 132.2, 128.2, 113.6, 111.4, 85.3, 63.3, 55.6, 55.3, 54.9, 48.9, 41.8, 35.1, 33.1, 28.2, 28.1, 27.9, 22.1, 19.2; HRMS (ESI-TOF): Calcd for $\mathrm{C}_{23} \mathrm{H}_{35} \mathrm{O}_{3}(\mathrm{M}+1)^{+} \mathrm{m} / z$ 359.2586, found $\mathrm{m} / z$ 359.2585; [a $]^{\mathbf{2 0}}{ }_{\mathbf{D}}^{-}$ $56.4\left(c 0.50, \mathrm{CHCl}_{3}\right)$.

1-((((1R,2S,3R)-2-(2,2-dimethylpent-4-en-1-yl)-1-methyl-3-(prop-1-en-2-yl)cyclopentyl)oxy) methyl)-4methoxybenzene

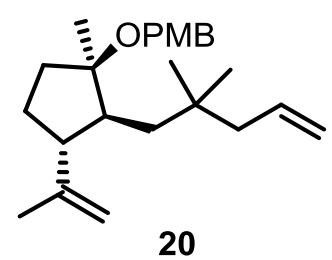

To a suspension of methyltriphenylphosphonium bromide (1.14 g, $2.00 \mathrm{eq}, 3.18 \mathrm{mmol})$ in THF (16 mL) was added KHMDS solution ( $3.18 \mathrm{~mL}, 2.00 \mathrm{eq}, 3.18 \mathrm{mmol}, 1 \mathrm{M}$ solution in THF) dropwise at $0{ }^{\circ} \mathrm{C}$. The resulting yellow solution was stirred for additional $30 \mathrm{~min}$ and then treated with a solution of aldehyde 26 (570 mg, $1.59 \mathrm{mmol})$ in THF $(1.6 \mathrm{~mL})$. The reaction mixture was stirred for $1 \mathrm{~h}$ and then quenched with sat. aq. $\mathrm{NH}_{4} \mathrm{Cl}$ solution $(5 \mathrm{~mL})$. The organic layer was washed with brine, dried $\left(\mathrm{MgSO}_{4}\right)$ and evaporated. The crude product was purified by column chromatography (PE:MTBE, 99:1) to afford olefin $\mathbf{2 0}$ as colorless oil (505 $\mathrm{mg}, 89 \%$ ). ${ }^{1} \mathbf{H}$ NMR $\left(400 \mathrm{MHz}, \mathbf{C D C l}_{3}\right) \boldsymbol{\delta} 7.26(\mathrm{~d}, J=8.7 \mathrm{~Hz}, 2 \mathrm{H}), 6.88(\mathrm{~d}, J=8.7 \mathrm{~Hz}, 2 \mathrm{H}), 5.84(\mathrm{ddt}, J=17.6,10.3$, $7.4 \mathrm{~Hz}, 1 \mathrm{H}), 5.05-4.93(\mathrm{~m}, 2 \mathrm{H}), 4.76(\mathrm{~d}, J=2.2 \mathrm{~Hz}, 1 \mathrm{H}), 4.69(\mathrm{dd}, J=2.3,1.3 \mathrm{~Hz}, 1 \mathrm{H}), 4.38(\mathrm{~d}, J=11.4 \mathrm{~Hz}$, $1 \mathrm{H}), 4.32(\mathrm{~d}, J=11.4 \mathrm{~Hz}, 1 \mathrm{H}), 3.81(\mathrm{~s}, 3 \mathrm{H}), 2.65-2.51(\mathrm{~m}, 1 \mathrm{H}), 2.10-2.03(\mathrm{~m}, 1 \mathrm{H}), 1.97-1.82(\mathrm{~m}, 4 \mathrm{H})$, $1.71(\mathrm{~s}, 3 \mathrm{H}), 1.60(\mathrm{ddd}, J=9.0,5.3,3.4 \mathrm{~Hz}, 1 \mathrm{H}), 1.53-1.41(\mathrm{~m}, 2 \mathrm{H}), 1.30(\mathrm{~s}, 3 \mathrm{H}), 1.18(\mathrm{dd}, J=15.0,5.4 \mathrm{~Hz}$, 1H), $1.02-0.82(\mathrm{~m}, 2 \mathrm{H}), 0.81(\mathrm{~s}, 3 \mathrm{H}), 0.78(\mathrm{~s}, 3 \mathrm{H}) .{ }^{13} \mathbf{C}$ NMR (101 MHz, $\left.\mathbf{C D C l}_{3}\right) \boldsymbol{\delta} 158.6,148.2,136.3$, 132.5, 128.0, 116.3, 113.6, 110.8, 85.6, 63.1, 55.3, 54.9, 48.9, 47.9, 41.1, 35.0, 32.8, 28.2, 27.2, 27.1, 22.0,

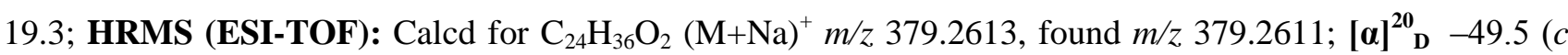
$\left.1.31, \mathrm{CHCl}_{3}\right)$. 


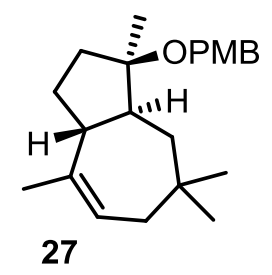

A solution of olefin $20(481 \mathrm{mg}, 1.35 \mathrm{mmol})$ and Grubbs' $2^{\text {nd }}$ generation $(57.3 \mathrm{mg}, 67.5 \mu \mathrm{mol})$ in toluene $(13.5 \mathrm{~mL})$ was stirred for at room temperature $15 \mathrm{~min}$ and then heated to reflux for $1 \mathrm{~h}$. After being cooled down to room temperature, the volatiles were removed and the obtained crude product purified by flash chromatography (PE/MTBE, 99:1) to afford compound 27 as colorless oil (361 mg, 81\%). ${ }^{\mathbf{1}} \mathbf{H}$ NMR (400 $\left.\mathbf{M H z}, \mathbf{C D C l}_{3}\right) \boldsymbol{\delta} 7.25(\mathrm{~d}, J=8.8 \mathrm{~Hz}, 2 \mathrm{H}), 6.88(\mathrm{~d}, J=8.7 \mathrm{~Hz}, 2 \mathrm{H}), 5.44-5.29(\mathrm{~m}, 1 \mathrm{H}), 4.41-4.28(\mathrm{~m}, 2 \mathrm{H})$, $3.81(\mathrm{~s}, 3 \mathrm{H}), 2.93(\mathrm{dd}, J=17.4,9.3 \mathrm{~Hz}, 1 \mathrm{H}), 2.14(\mathrm{dd}, J=14.2,4.8 \mathrm{~Hz}, 1 \mathrm{H}), 2.06(\mathrm{ddd}, J=13.3,7.6,3.8 \mathrm{~Hz}$, $1 \mathrm{H}), 1.87-1.70(\mathrm{~m}, 6 \mathrm{H}), 1.66(\mathrm{~d}, J=12.0 \mathrm{~Hz}, 1 \mathrm{H}), 1.58(\mathrm{dt}, J=13.5,2.4 \mathrm{~Hz} 1 \mathrm{H}), 1.50-1.33(\mathrm{~m}, 2 \mathrm{H}), 1.23$ (s, 3H), 0.95 (s, 3H), 0.84 (s, 3H); ${ }^{13} \mathbf{C}$ NMR (101 MHz, $\left.\mathbf{C D C l}_{3}\right) \boldsymbol{\delta}$ 158.5, 133.4, 142.1, 128.0, 122.8, 113.6, 84.7, 63.3, 55.3, 50.8, 45.6, 43.3, 40.4, 35.3, 34.0, 31.1, 25.4, 24.8, 22.1, 21.0; HRMS (ESI-TOF): Calcd for $\mathrm{C}_{22} \mathrm{H}_{32} \mathrm{O}_{2}(\mathrm{M}+\mathrm{Na})^{+} \mathrm{m} / \mathrm{z} 351.2300$, found $\mathrm{m} / \mathrm{z} 351.2297 ;[\boldsymbol{\alpha}]^{\mathbf{2 0}}{ }_{\mathbf{D}}-37.7\left(c 0.48, \mathrm{CHCl}_{3}\right)$.

\section{(1R,3aR,8aS)-1,4,7,7-tetramethyl-1,2,3,3a,6,7,8,8a-octahydroazulen-1-ol}

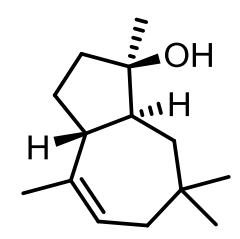

19

To a solution of compound $27(296 \mathrm{mg}, 0.900 \mathrm{mmol})$ in a mixture of $\mathrm{CH}_{2} \mathrm{Cl}_{2} / \mathrm{pH} 7$ buffer solution $(9.9 \mathrm{~mL}$, 10:1) was added DDQ (409 mg, $2.00 \mathrm{eq}, 1.80 \mathrm{mmol}$ ) at once. The mixture was allowed to stir for $30 \mathrm{~min}$ and then quenched with sat. aq. $\mathrm{NaHCO}_{3}$ solution $(2 \mathrm{~mL})$. The reaction mixture was extracted with MTBE and the combined organic layers were washed with brine, dried $\left(\mathrm{MgSO}_{4}\right)$ and concentrated. The obtained crude product was purified by flash chromatography (PE:MTBE, 9:1) to afford alcohol 19 as colorless oil (133 mg, 71\%). ${ }^{1} \mathbf{H}$ NMR (400 MHz, $\mathbf{C D C l}_{3}$ ) $\boldsymbol{\delta} 5.44-5.32(\mathrm{~m}, 1 \mathrm{H}), 2.79$ (dd, $\left.J=16.3,8.4 \mathrm{~Hz}, 1 \mathrm{H}\right), 2.11$ (dd, $J=14.4$, $5.4 \mathrm{~Hz}, 1 \mathrm{H}), 1.89$ (tdd, $J=8.9,6.5,3.1 \mathrm{~Hz}, 1 \mathrm{H}), 1.79$ (ddd, $J=14.4,8.4,2.0 \mathrm{~Hz}, 1 \mathrm{H}), 1.74-1.66(\mathrm{~m}, 6 \mathrm{H})$, $1.55(\mathrm{dt}, J=12.3,1.9 \mathrm{~Hz}, 1 \mathrm{H}), 1.42(\mathrm{t}, J=12.2 \mathrm{~Hz}, 1 \mathrm{H}), 1.34(\mathrm{ddd}, J=12.1,8.0,2.0 \mathrm{~Hz}, 1 \mathrm{H}), 1.22(\mathrm{~s}, 3 \mathrm{H})$, 0.96 (s, 3H), 0.84 (s, 3H); ${ }^{13} \mathbf{C}$ NMR (101 MHz, $\left.\mathbf{C D C l}_{\mathbf{3}}\right) \boldsymbol{\delta}$ 141.5, 122.8, 80.7, 48.7, 45.9, 43.8, 40.9, 40.4, $33.9,31.1,26.5,25.4,24.9,22.2 ;[\boldsymbol{\alpha}]^{\mathbf{2 0}}{ }_{\mathrm{D}}+28.2\left(\mathrm{c} 0.38, \mathrm{CHCl}_{3}\right)$. 


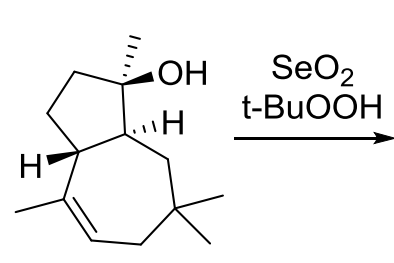

19

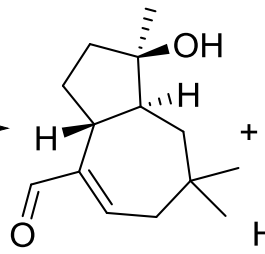

$2835 \%$

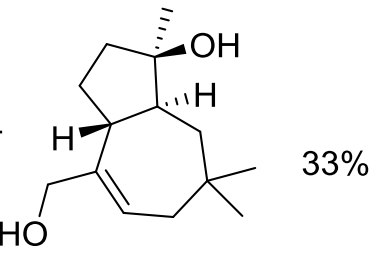

$22 \%$

29

$\mathrm{NaBH}_{4}, \mathrm{MeOH} \uparrow$

To a suspension of $\mathrm{SeO}_{2}(31.6 \mathrm{mg}, 0.5 \mathrm{eq}, 0.285 \mathrm{mmol})$ in $\mathrm{CH}_{2} \mathrm{Cl}_{2}$ was added a solution of $t$ - $\mathrm{BuOOH}(215 \mu \mathrm{L}$, $2.00 \mathrm{eq}, 1.18 \mathrm{mmol}$, ca. $5.5 \mathrm{M}$ in decane). The reaction mixture was stirred for $30 \mathrm{~min}$ and then treated with a solution of compound $19(123 \mathrm{mg}, 590 \mu \mathrm{mol})$ in $\mathrm{CH}_{2} \mathrm{Cl}_{2}(0.9 \mathrm{~mL})$. After complete starting material (30 min, by TLC), the reaction was quenched with sat. aq. $\mathrm{Na}_{2} \mathrm{SO}_{3}$ solution $(2 \mathrm{~mL})$ and extracted with MTBE $(3 \times 5$ $\mathrm{mL})$. The combined organic layers were washed with brine and dried $\left(\mathrm{MgSO}_{4}\right)$. The obtained crude product was purified by column chromatography (PE/MTBE, 1:1) to afford alcohol 29 (29.0 mg, 22\%) and aldehyde $28(46.0 \mathrm{mg}, 35 \%)$ as colorless oils. The aldehyde 28 was further converted into the alcohol 29 with $\mathrm{NaBH}_{4}$ in MeOH. Analytical data of alcohol 29; ${ }^{1} \mathbf{H}$ NMR (400 MHz, $\left.\mathbf{C D C l}_{3}\right) \boldsymbol{\delta} 5.72-5.55$ (m, 1H), 4.11 (s, 2H), 2.86 $(\mathrm{q}, J=8.3 \mathrm{~Hz}, 1 \mathrm{H}), 2.16(\mathrm{dd}, J=14.3,5.4 \mathrm{~Hz}, 1 \mathrm{H}), 2.03-1.81(\mathrm{~m}, 3 \mathrm{H}), 1.80-1.67(\mathrm{~m}, 2 \mathrm{H}), 1.58(\mathrm{~d}, J=12.4$ $\mathrm{Hz}, 1 \mathrm{H}), 1.49-1.35(\mathrm{~m}, 3 \mathrm{H}), 1.25-1.21(\mathrm{~m}, 1 \mathrm{H}), 1.22(\mathrm{~s}, 3 \mathrm{H}), 0.99(\mathrm{~s}, 3 \mathrm{H}), 0.86(\mathrm{~s}, 3 \mathrm{H}) ;{ }^{\mathbf{1 3}} \mathbf{C} \mathbf{~ N M R}(\mathbf{1 0 1}$ MHz, $\left.\mathbf{C D C l}_{3}\right) \boldsymbol{\delta} 144.7,126.3,80.6,66.7,49.5,44.4,43.7,40.8,40.3,34.0,30.9,26.4,25.2$, 23.9; HRMS (ESI-TOF): Calcd for $\mathrm{C}_{14} \mathrm{H}_{24} \mathrm{O}_{2}(\mathrm{M}+\mathrm{Na})^{+} \mathrm{m} / \mathrm{z} 247.1674$, found $\mathrm{m} / \mathrm{z} 247.1674 ;[\mathbf{\alpha}]^{\mathbf{2 0}}{ }_{\mathbf{D}}+35.8\left(c 0.43, \mathrm{CHCl}_{3}\right)$.

(+)-Pyxidatol C (2)

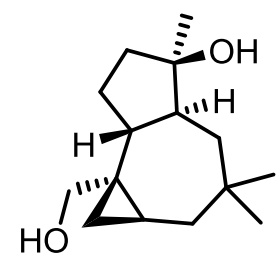

2

To a solution of diol $29(13.2 \mathrm{mg}, 0.0590 \mathrm{mmol})$ in $\mathrm{CH}_{2} \mathrm{Cl}_{2}(1 \mathrm{~mL})$ was added diethyl zinc $(0.590 \mathrm{~mL}, 10 \mathrm{eq}$, $0.590 \mathrm{~mL}, 1 \mathrm{M}$ solution in hexane) at $-30{ }^{\circ} \mathrm{C}$ and stirred for $10 \mathrm{~min}$. Later, $\mathrm{CH}_{2} \mathrm{I}_{2}(95.2 \mu 1,20 \mathrm{eq}, 1.18 \mathrm{mmol})$ was added into the reaction mixture at the same temperature and allowed to reach $0{ }^{\circ} \mathrm{C}$ for $2 \mathrm{~h}$. Then, the cooling bath was removed and the mixture was stirred at $\mathrm{rt}$ for overnight. The reaction was quenched with water and extracted with MTBE. The combined organic layers were washed with $2 \mathrm{~N} \mathrm{NaOH}$ solution, brine and dried $\left(\mathrm{MgSO}_{4}\right)$. The obtained crude product after evaporation of solvent was purified by column chromatography (PE:MTBE, 1:1) to afford (+)-pyxidatol C (2) as light yellow oil (4.6 mg, 33\%). HRMS 
(ESI-TOF): Calcd for $\mathrm{C}_{15} \mathrm{H}_{26} \mathrm{O}_{2}(\mathrm{M}+\mathrm{Na})^{+} \mathrm{m} / \mathrm{z}: 261.1831$, found $\mathrm{m} / \mathrm{z} 261.1831 ;[\boldsymbol{\alpha}]^{\mathbf{2 0}}{ }_{\mathbf{D}}+8.0(c 0.20, \mathrm{MeOH})$; For ${ }^{1} \mathrm{H}$ and ${ }^{13} \mathrm{C}$ NMR, see comparison Tables 3 and 4 below. 
Comparison Table-3: ( ${ }^{1} \mathrm{H}$ NMR characterization data of pyxidatol C (2))

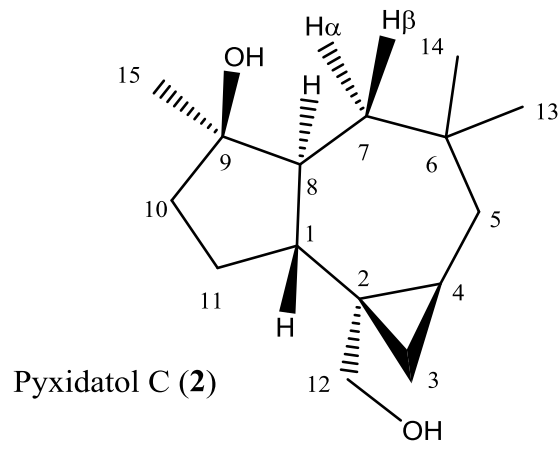

\begin{tabular}{|l|l|l|l|}
\hline $\begin{array}{c}{ }^{\mathrm{l}} \mathrm{H} \\
\mathrm{NMR}\end{array}$ & $\begin{array}{l}\text { Natural pyxidatol C } \\
\left(600 \mathrm{MHz},\left(\mathrm{CD}_{3}\right)_{2} \mathrm{CO}\right)\end{array}$ & $\begin{array}{l}\text { Synthetic pyxidatol C } \\
\left(400 \mathrm{MHz},\left(\mathrm{CD}_{3}\right)_{2} \mathrm{CO}\right)\end{array}$ & $\begin{array}{l}\text { Our synthetic pyxidatol C } \\
\left(\mathbf{4 0 0} \mathbf{M H z},\left(\mathrm{CD}_{3}\right)_{2} \mathrm{CO}\right)\end{array}$ \\
\hline 1 & $1.70($ overlap$)$ & $1.64-1.69(\mathrm{~m})$ & $1.69-1.64(\mathrm{~m})$ \\
\hline $3 \alpha$ & $0.14(\mathrm{t}, J=3.8 \mathrm{~Hz})$ & $0.14(\mathrm{t}, J=4.1 \mathrm{~Hz})$ & $0.15(\mathrm{t}, J=4.2 \mathrm{~Hz})$ \\
\hline $3 \beta$ & $0.70(\mathrm{dd}, J=8.5,3.8 \mathrm{~Hz})$ & $0.69(\mathrm{dd}, J=8.5,3.7 \mathrm{~Hz})$ & $0.69(\mathrm{dd}, J=8.5,3.7 \mathrm{~Hz})$ \\
\hline 4 & $0.73-0.78(\mathrm{~m})$ & $0.72-0.79(\mathrm{~m})$ & $0.80-0.72(\mathrm{~m})$ \\
\hline $5 \alpha$ & $1.11-1.14(\mathrm{~m})$ & $1.10(\mathrm{dd}, J=10.1,4.3 \mathrm{~Hz})$ & $1.15-1.08(\mathrm{~m})$ \\
\hline $5 \beta$ & $1.85($ overlap$)$ & $1.81-1.85(\mathrm{~m})$ & $1.86-1.84(\mathrm{~m})$ \\
\hline $7 \alpha$ & $1.17-1.24(\mathrm{~m})$ & $1.15-1.22(\mathrm{~m})$ & $1.21-1.16(\mathrm{~m})$ \\
\hline $7 \beta$ & $1.42-1.45(\mathrm{~m})$ & $1.41-1.45(\mathrm{~m})$ & $1.44(\mathrm{dt}, J=13.4,2.5 \mathrm{~Hz})$ \\
\hline 8 & $1.98($ overlap$)$ & $1.92-2.00(\mathrm{~m})$ & $2.01-1.92(\mathrm{~m})$ \\
\hline $10 \alpha$ & $1.61-1.64(\mathrm{~m})$ & $1.61-1.63(\mathrm{~m})$ & $1.63-1.59(\mathrm{~m})$ \\
\hline $10 \beta$ & $1.72($ overlap$)$ & $1.69-1.73(\mathrm{~m})$ & $1.74-1.69(\mathrm{~m})$ \\
\hline $11 \alpha$ & $1.82($ overlap$)$ & $1.78-1.81(\mathrm{~m})$ & $1.83-1.78(\mathrm{~m})$ \\
\hline $11 \beta$ & $1.96($ overlap$)$ & $1.92-2.00(\mathrm{~m})$ & $2.01-1.92(\mathrm{~m})$ \\
\hline $12 \alpha$ & $3.49(\mathrm{dd}, J=11.2,5.0 \mathrm{~Hz})$ & $3.48(\mathrm{dd}, J=11.1,5.6 \mathrm{~Hz})$ & $3.49(\mathrm{dd}, J=11.2,5.8 \mathrm{~Hz})$ \\
\hline $12 \beta$ & $3.61(\mathrm{dd}, J=11.2,5.0 \mathrm{~Hz})$ & $3.59(\mathrm{dd}, J=11.1,4.6 \mathrm{~Hz})$ & $3.60(\mathrm{dd}, J=11.2,4.8 \mathrm{~Hz})$ \\
\hline 13 & $1.07(\mathrm{~s}, 3 \mathrm{H})$ & $1.04(\mathrm{~s}, 3 \mathrm{H})$ & $1.03(\mathrm{~s}, 3 \mathrm{H})$ \\
\hline 14 & $0.88(\mathrm{~s}, 3 \mathrm{H})$ & $0.89(\mathrm{~s}, 3 \mathrm{H})$ & $0.90(\mathrm{~s}, 3 \mathrm{H})$ \\
\hline 15 & $1.19(\mathrm{~s}, 3 \mathrm{H})$ & $1.19(\mathrm{~s}, 3 \mathrm{H})$ & $1.19(\mathrm{~s}, 3 \mathrm{H})$ \\
\hline $9(\mathrm{OH})$ & $2.80(\mathrm{~s})$ & $2.79(\mathrm{~s})$ & $2.80(\mathrm{~s})$ \\
\hline $12(\mathrm{OH})$ & $3.42(\mathrm{t}, J=5.0 \mathrm{~Hz})$ & $3.42(\mathrm{t}, J=5.0 \mathrm{~Hz})$ & $3.42(\mathrm{t}, J=5.0 \mathrm{~Hz})$ \\
\hline
\end{tabular}


Comparison Table-4: $\left({ }^{13} \mathrm{C}\right.$ NMR characterization data of pyxidatol C (2))

\begin{tabular}{|c|c|c|c|c|}
\hline $13 \mathrm{C}$ & $\begin{array}{l}\text { Natural pyxidatol C } \\
\left(150 \mathrm{MHz},\left(\mathrm{CD}_{3}\right)_{2} \mathrm{CO}\right)\end{array}$ & $\begin{array}{l}\text { Synthetic pyxidatol C } \\
\left(100 \mathrm{MHz},\left(\mathrm{CD}_{3}\right)_{2} \mathrm{CO}\right)\end{array}$ & $\begin{array}{l}\text { Our synthetic pyxidatol C } \\
\left(100 \mathrm{MHz},\left(\mathrm{CD}_{3}\right)_{2} \mathrm{CO}\right)\end{array}$ & $\Delta \delta$ \\
\hline 1 & 48.2 & 49.2 & $\mathbf{4 9 . 1}$ & 0.9 \\
\hline 2 & 26.8 & 27.7 & $\mathbf{2 7 . 7}$ & 0.9 \\
\hline 3 & 19.8 & 20.7 & $\mathbf{2 0 . 7}$ & 0.9 \\
\hline 4 & 18.8 & 19.8 & $\mathbf{1 9 . 7}$ & 0.9 \\
\hline 5 & 43.2 & 44.1 & $\mathbf{4 4 . 1}$ & 0.9 \\
\hline 6 & 32.8 & 33.7 & $\mathbf{3 3 . 7}$ & 0.9 \\
\hline 7 & 43.7 & 44.7 & $\mathbf{4 4 . 6}$ & 0.9 \\
\hline 8 & 49.1 & 50.0 & $\mathbf{5 0 . 0}$ & 0.9 \\
\hline 9 & 79.3 & 80.3 & $\mathbf{8 0 . 2}$ & 0.9 \\
\hline 10 & 41.6 & 42.5 & $\mathbf{4 2 . 5}$ & 0.9 \\
\hline 11 & 23.0 & 24.0 & $\mathbf{2 3 . 9}$ & 0.9 \\
\hline 12 & 66.0 & 66.9 & $\mathbf{6 6 . 9}$ & 0.9 \\
\hline 13 & 23.7 & 24.6 & $\mathbf{2 4 . 6}$ & 0.9 \\
\hline 14 & 33.8 & 34.8 & $\mathbf{3 4 . 7}$ & 0.9 \\
\hline 15 & 25.4 & 26.4 & $\mathbf{2 6 . 3}$ & \\
\hline
\end{tabular}

Note: The $1 \mathrm{ppm}$ difference is due to different labeling on residual peak for $\left(\mathrm{CD}_{3}\right)_{2} \mathrm{CO}$ (the peak they labeled ${ }^{1}$ as 28.837 is very close to the residue peak of $\left(\mathrm{CD}_{3}\right)_{2} \mathrm{CO}$ which is referred as $\left.29.8 \mathrm{ppm}\right)$

\section{References:}

1. Earnshaw, C.; Wallis, C. J.; Warren, S. J. Chem. Soc., Perkin Trans.1 1979, 12, 3099.

2. Zheng, Y.-B.; Lu, C.-H.; Zheng, Z.-H.; Lin, X.-H.; Su, W.-J.; Shen, Y. -M. Helv. Chim. Acta 2008, 91 , 2174.

3. Zhou, L.; Yao, Y.; Xu, W.; Liang, G. J. Org. Chem. 2014, 79, 5345. 


\section{Spectroscopic data}

${ }^{1} \mathrm{H}$ NMR (200 MHz, $\mathrm{CDCl}_{3}$ ) of compound A

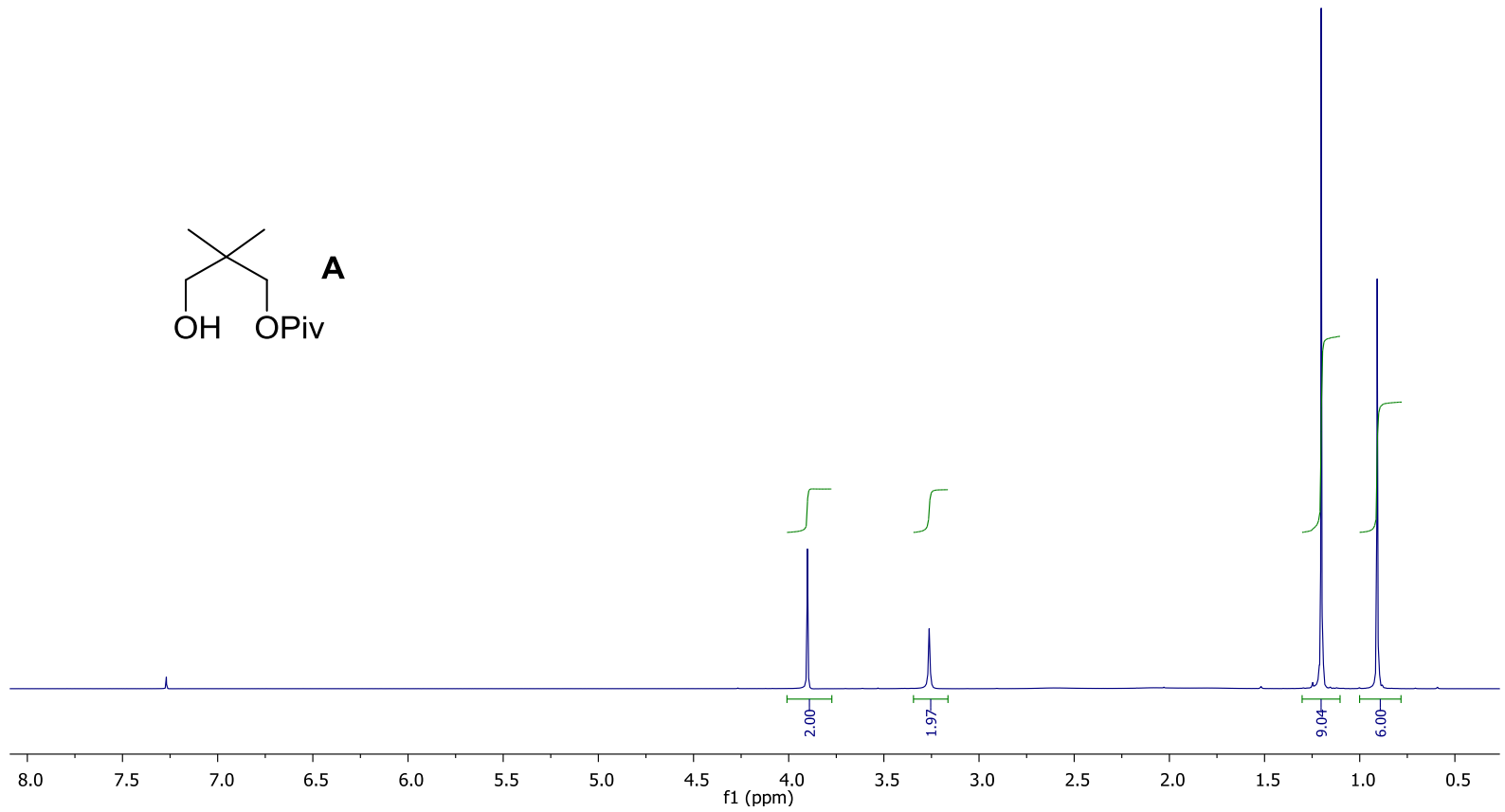

${ }^{13} \mathrm{C}$ NMR (101 MHz, $\mathrm{CDCl}_{3}$ ) of compound-A

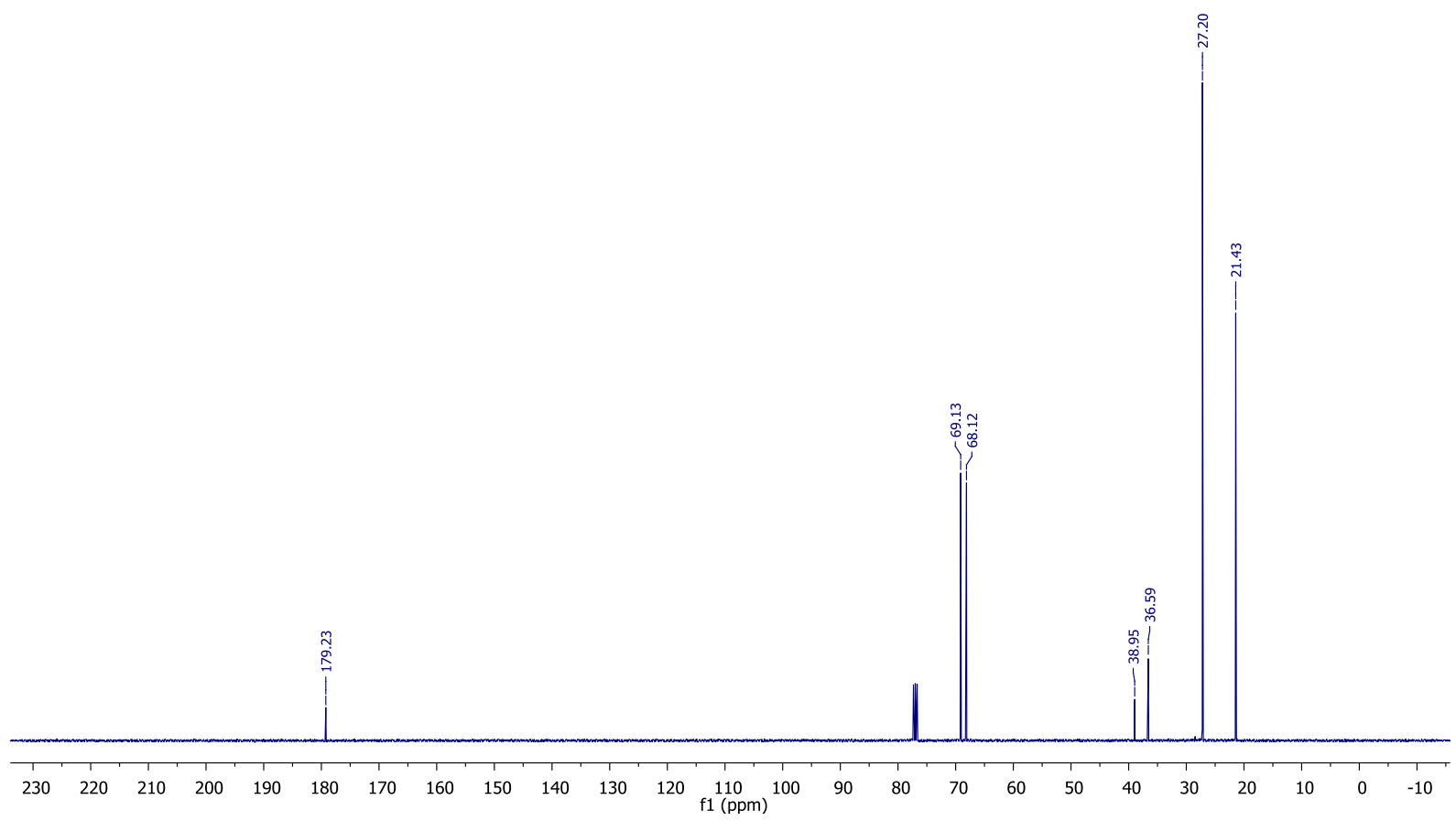


${ }^{1} \mathrm{H}$ NMR (200 MHz, $\mathrm{CDCl}_{3}$ ) of compound-10

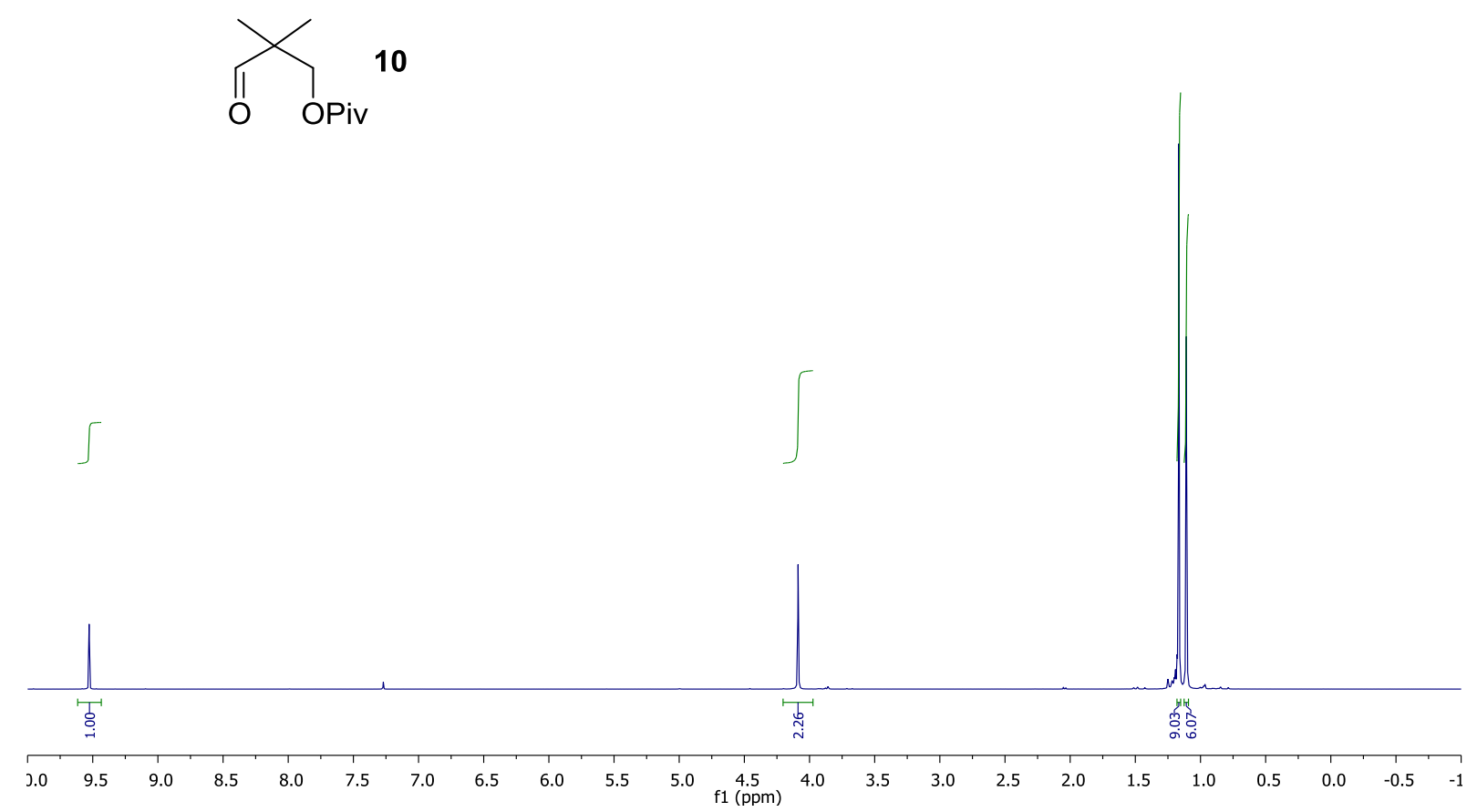

${ }^{13} \mathrm{C}$ NMR (101 MHz, $\mathrm{CDCl}_{3}$ ) of compound-10

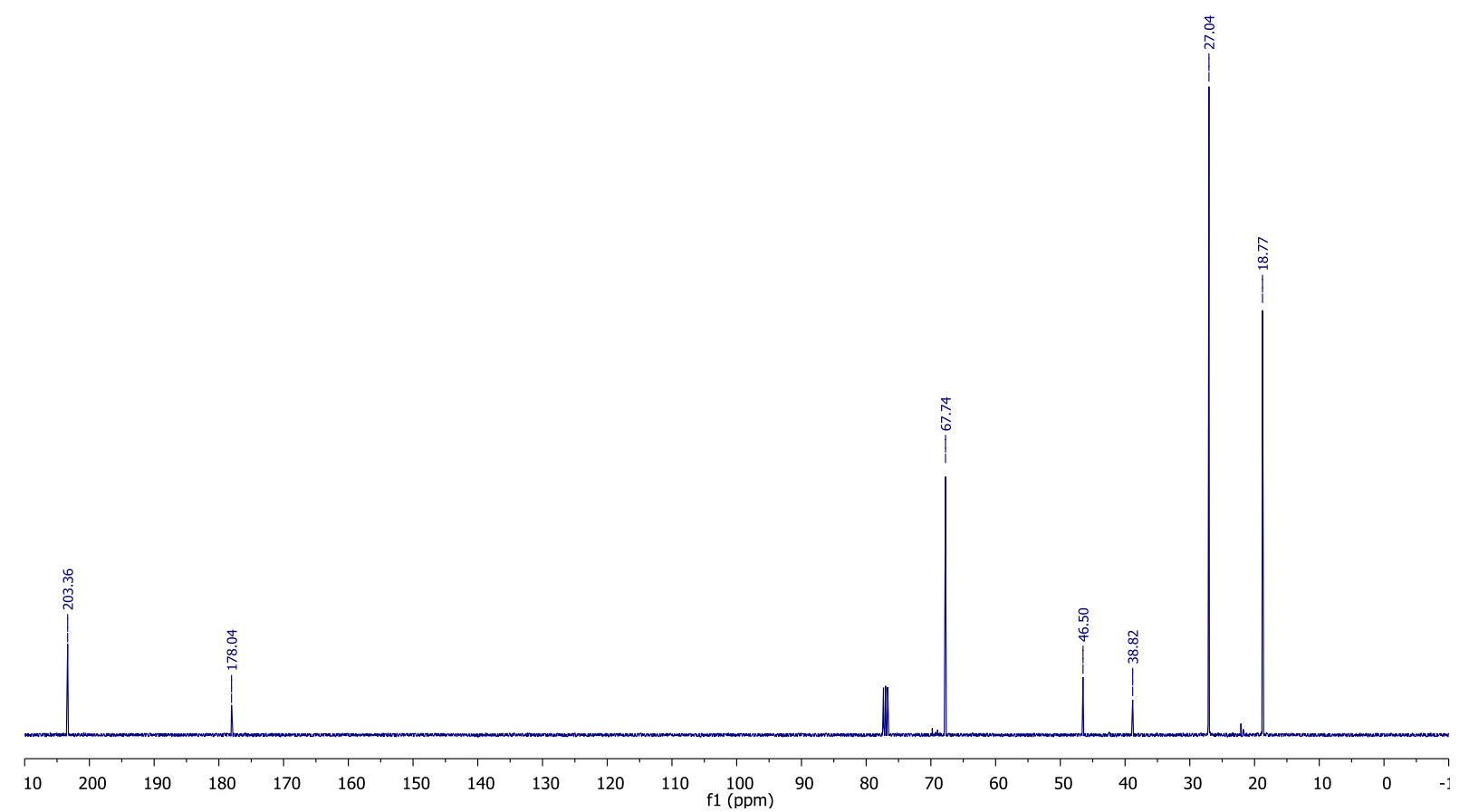


${ }^{1} \mathrm{H}$ NMR (400 MHz, $\mathrm{CDCl}_{3}$ ) of compound $\mathbf{1 1}$
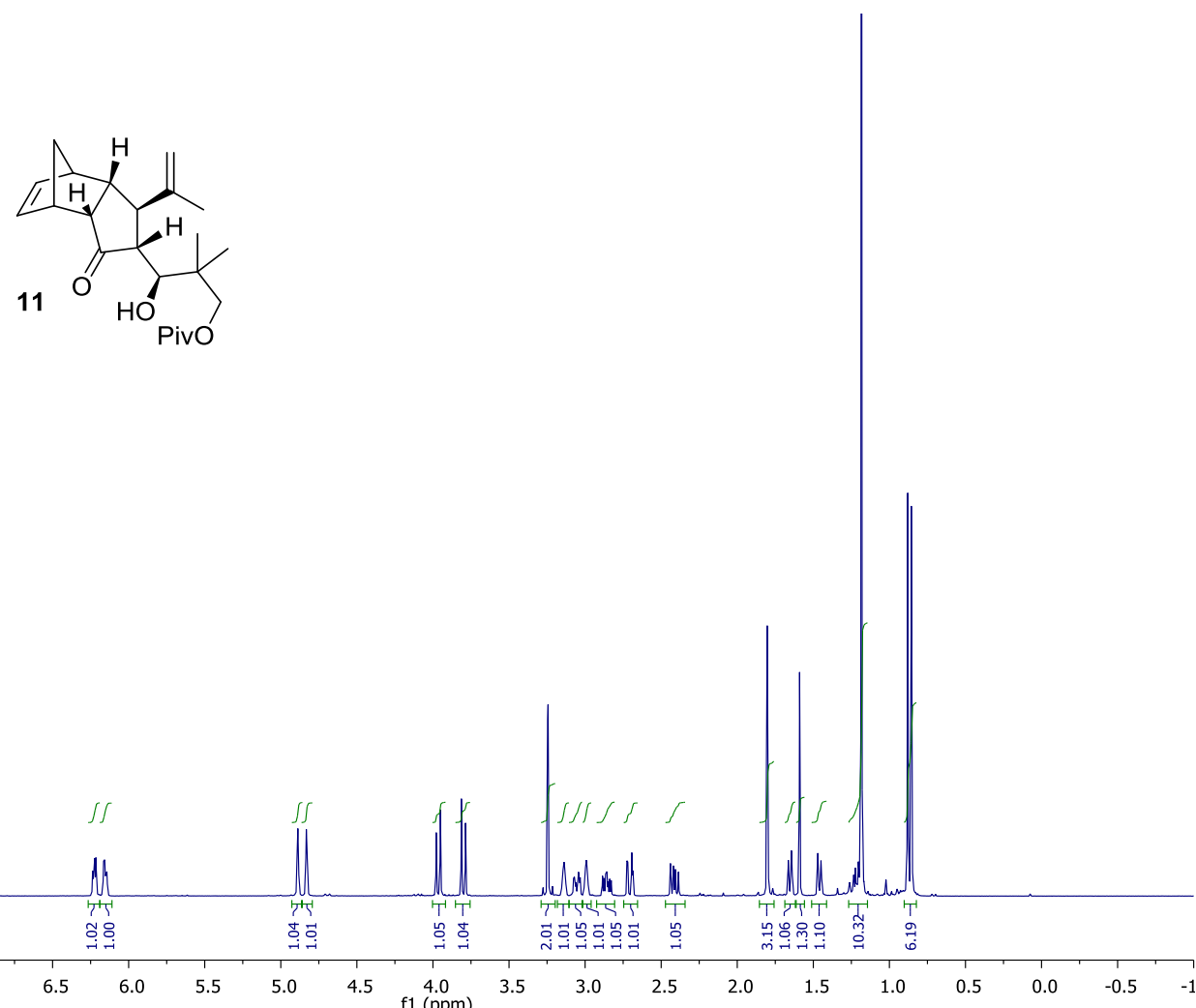

${ }^{13} \mathrm{C}$ NMR (101 MHz, $\mathrm{CDCl}_{3}$ ) of compound-11

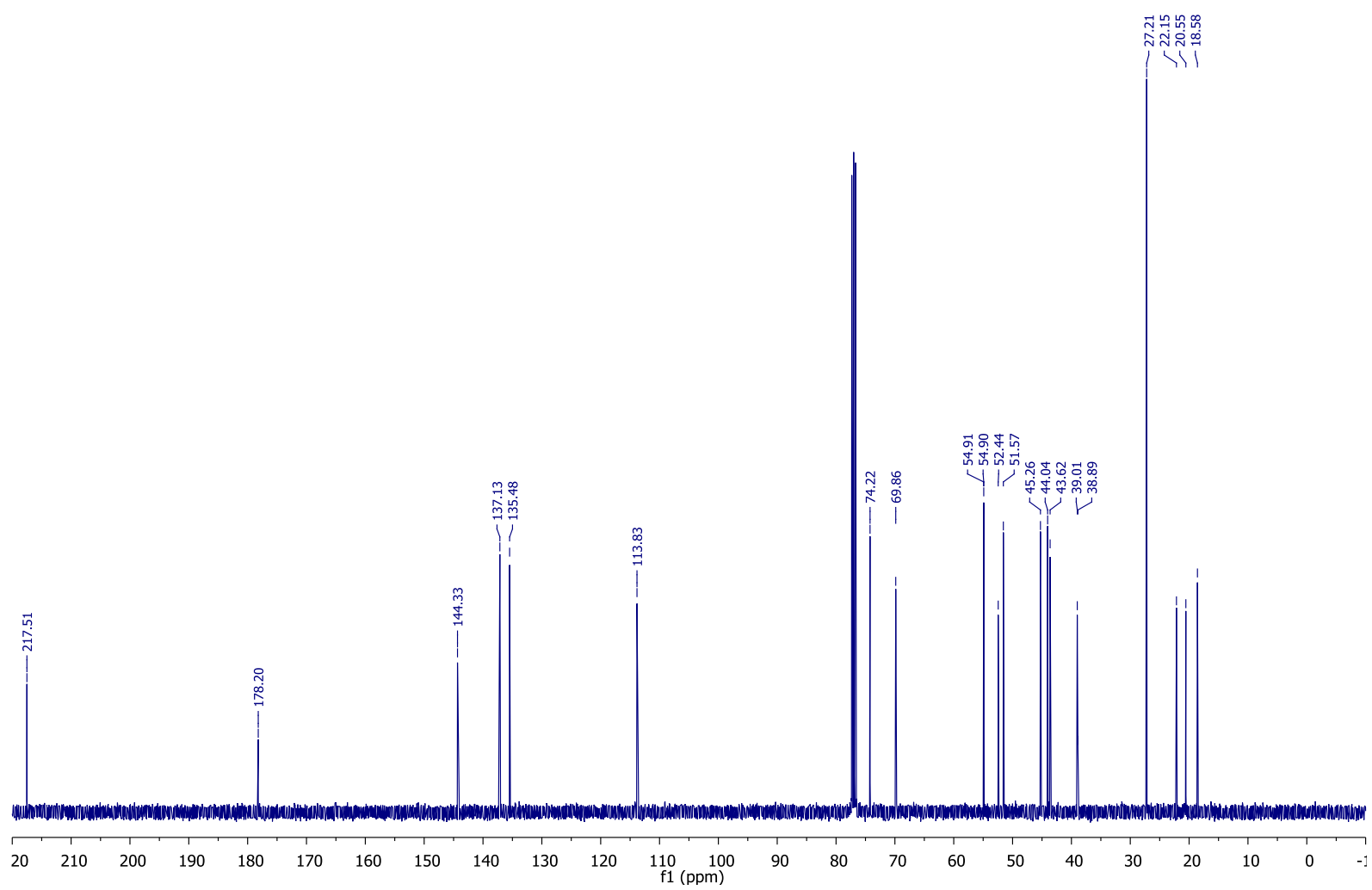


${ }^{1} \mathrm{H}$ NMR (400 MHz, $\mathrm{CDCl}_{3}$ ) of compound-12

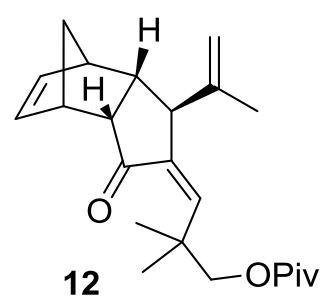

${ }^{13} \mathrm{C}$ NMR (101 MHz, $\mathrm{CDCl}_{3}$ ) of compound-12

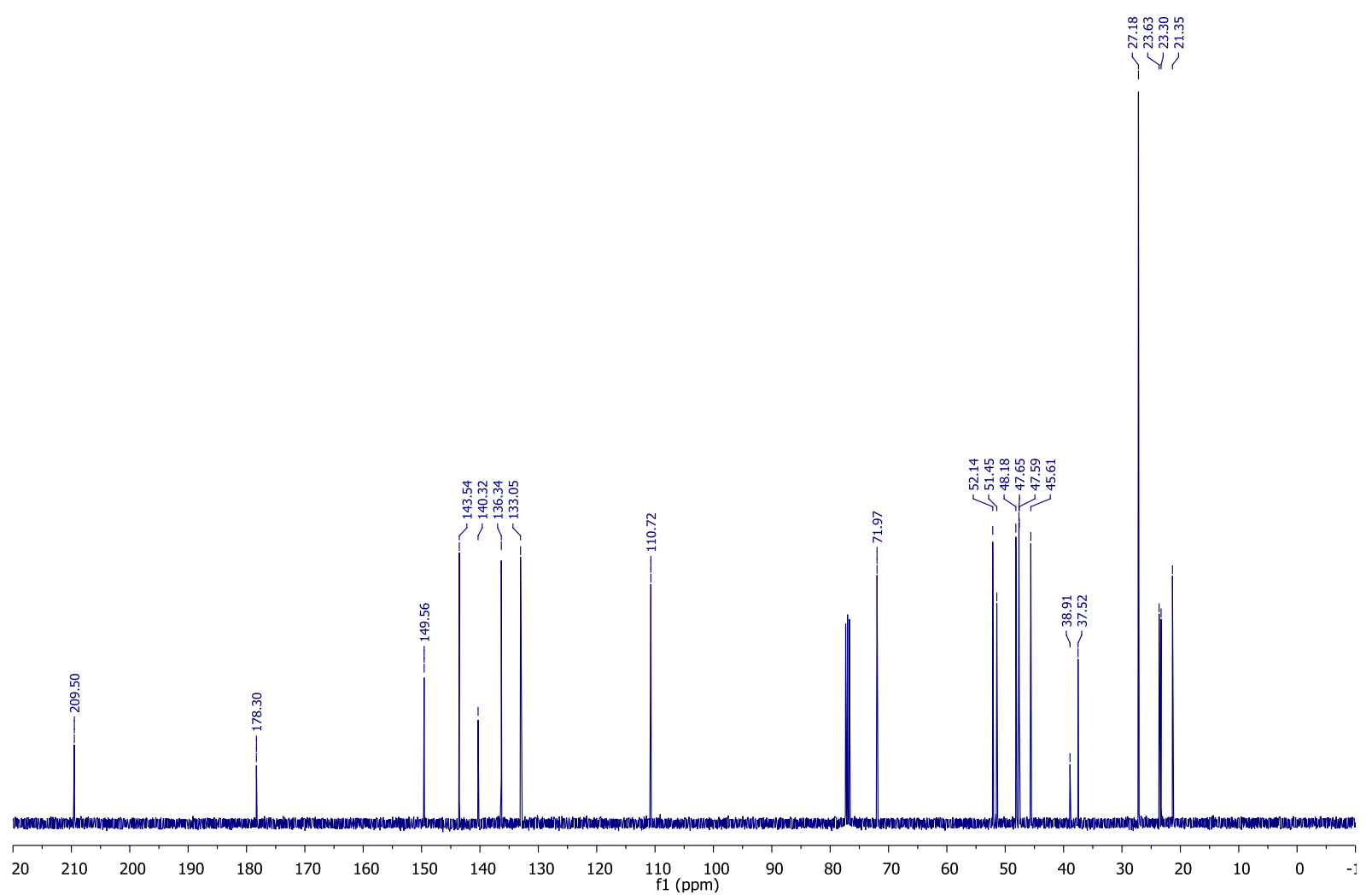


${ }^{1} \mathrm{H}$ NMR (400 MHz, $\mathrm{CDCl}_{3}$ of compound-14

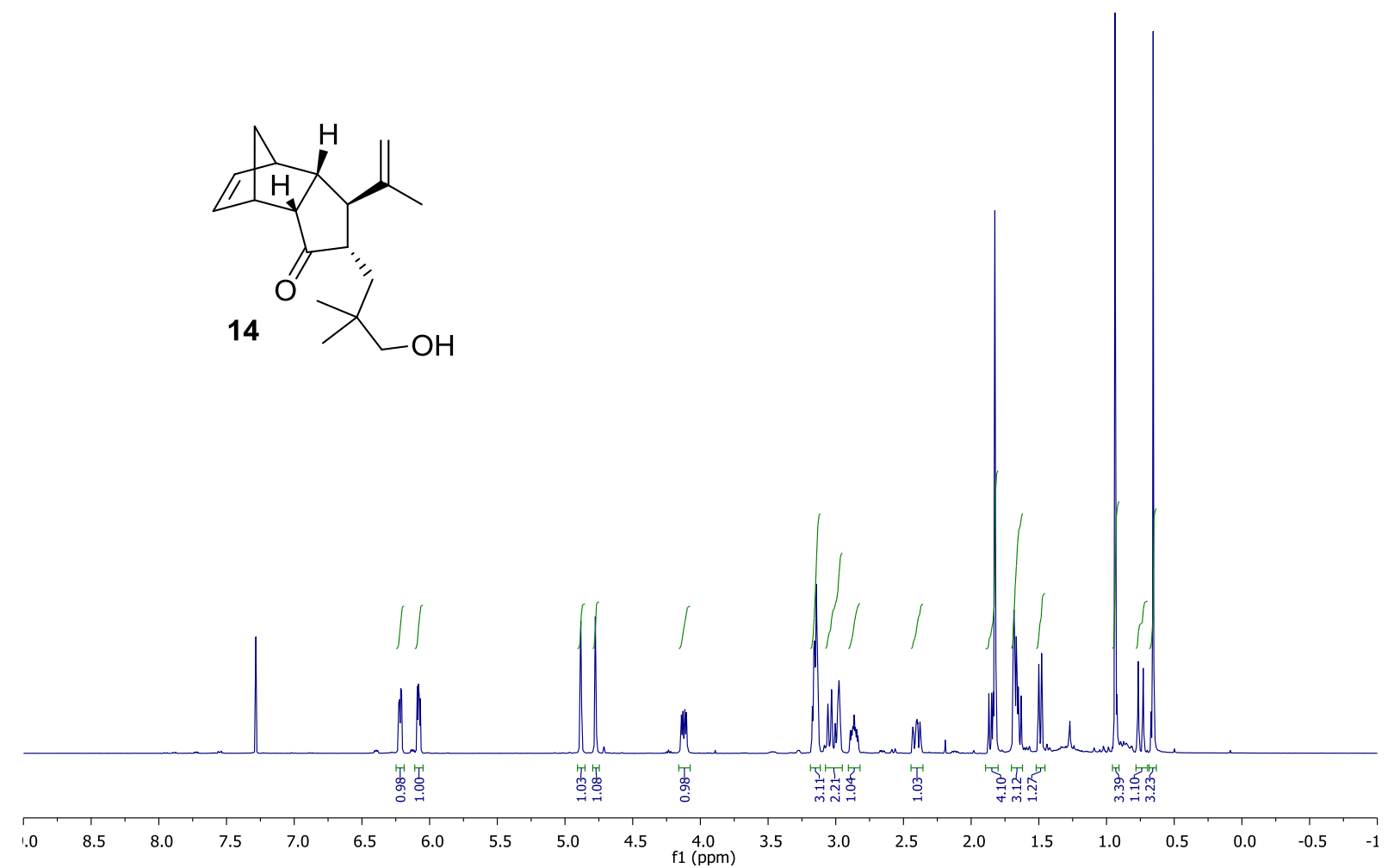

${ }^{13} \mathrm{C}$ NMR (101 MHz, $\mathrm{CDCl}_{3}$ ) of compound-14

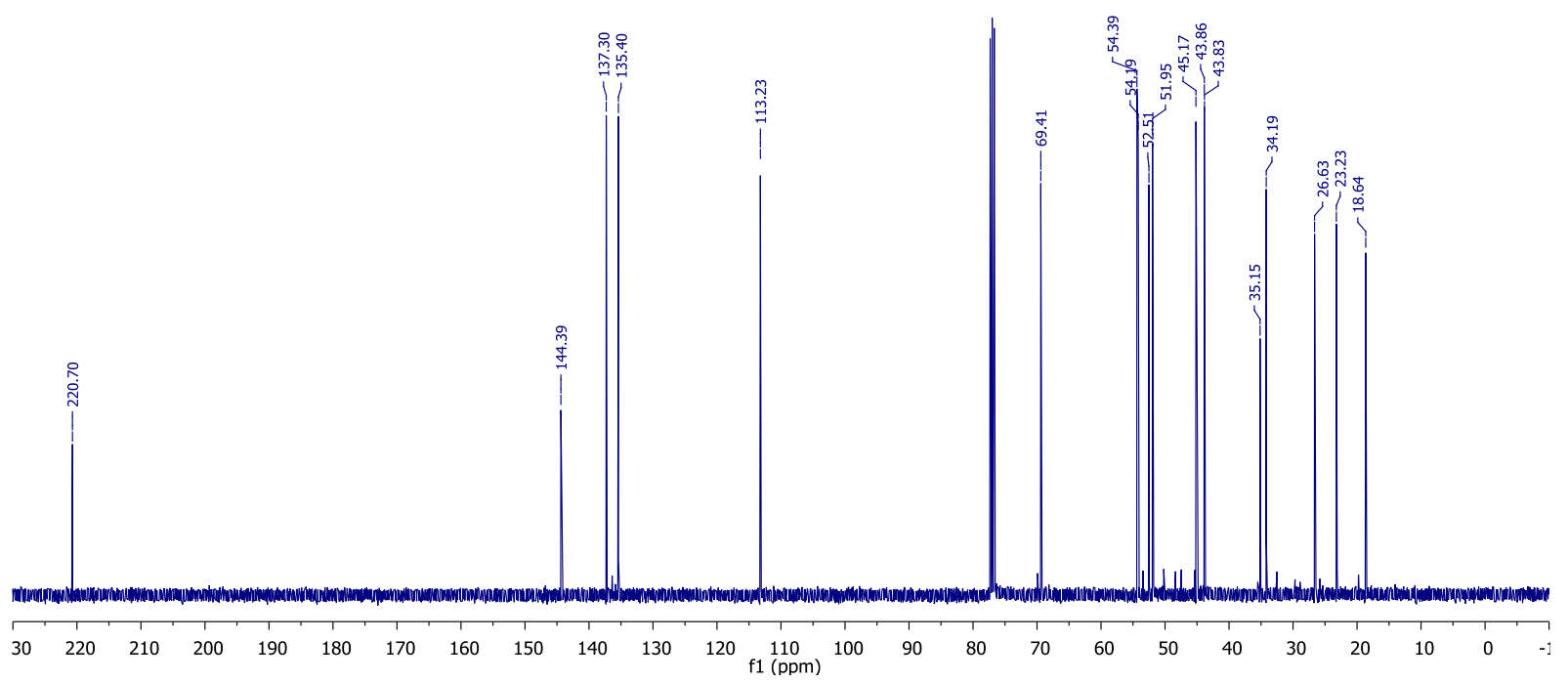


${ }^{1} \mathrm{H}$ NMR (500 MHz, $\mathrm{CDCl}_{3}$ ) of Compound-15

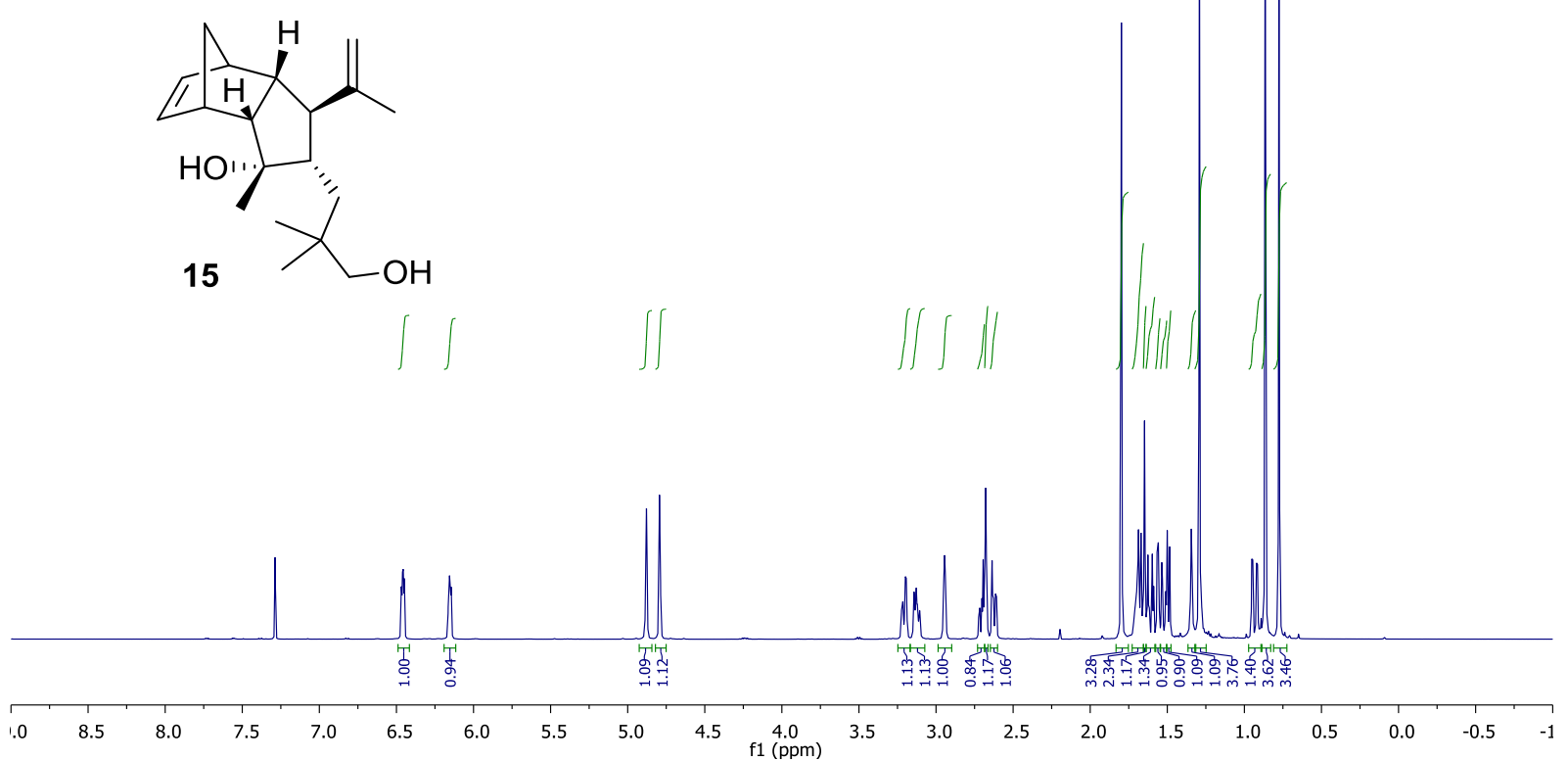

${ }^{13} \mathrm{C}$ NMR (101 MHz, $\mathrm{CDCl}_{3}$ ) of compound-15

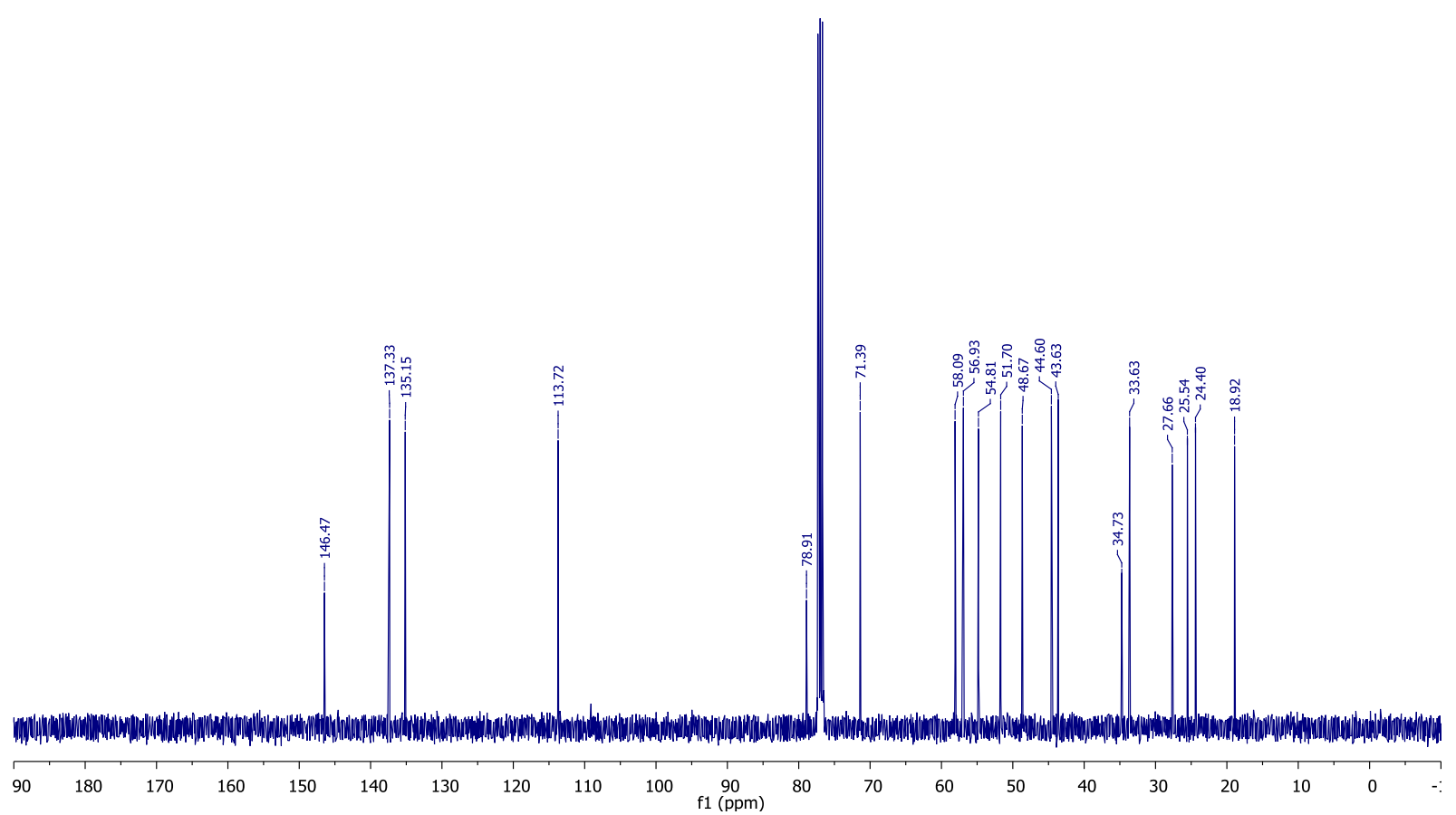


${ }^{1} \mathrm{H}$ NMR (400 MHz, $\mathrm{CDCl}_{3}$ ) of compound-6<smiles>C=C(C)[C@H]1C=C[C@](C)(O)[C@H]1CC(C)(C)CO</smiles>

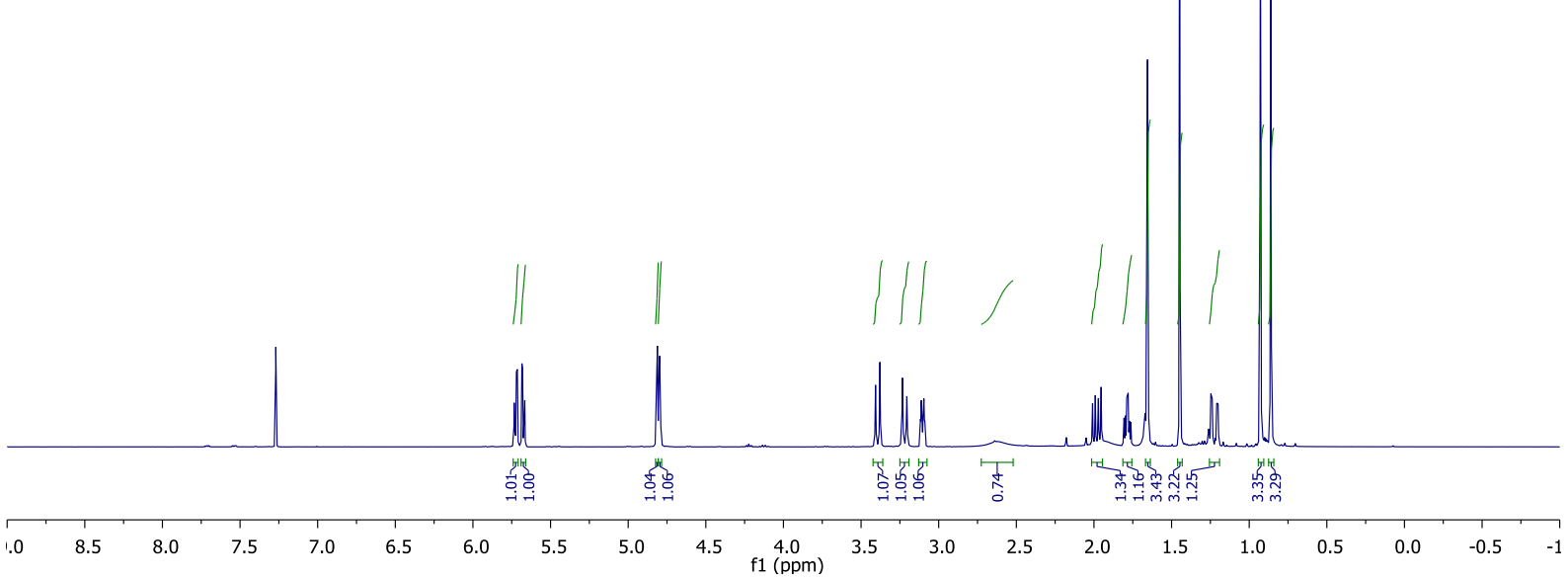

${ }^{13} \mathrm{C}$ NMR (101 MHz, $\mathrm{CDCl}_{3}$ ) of compound-6

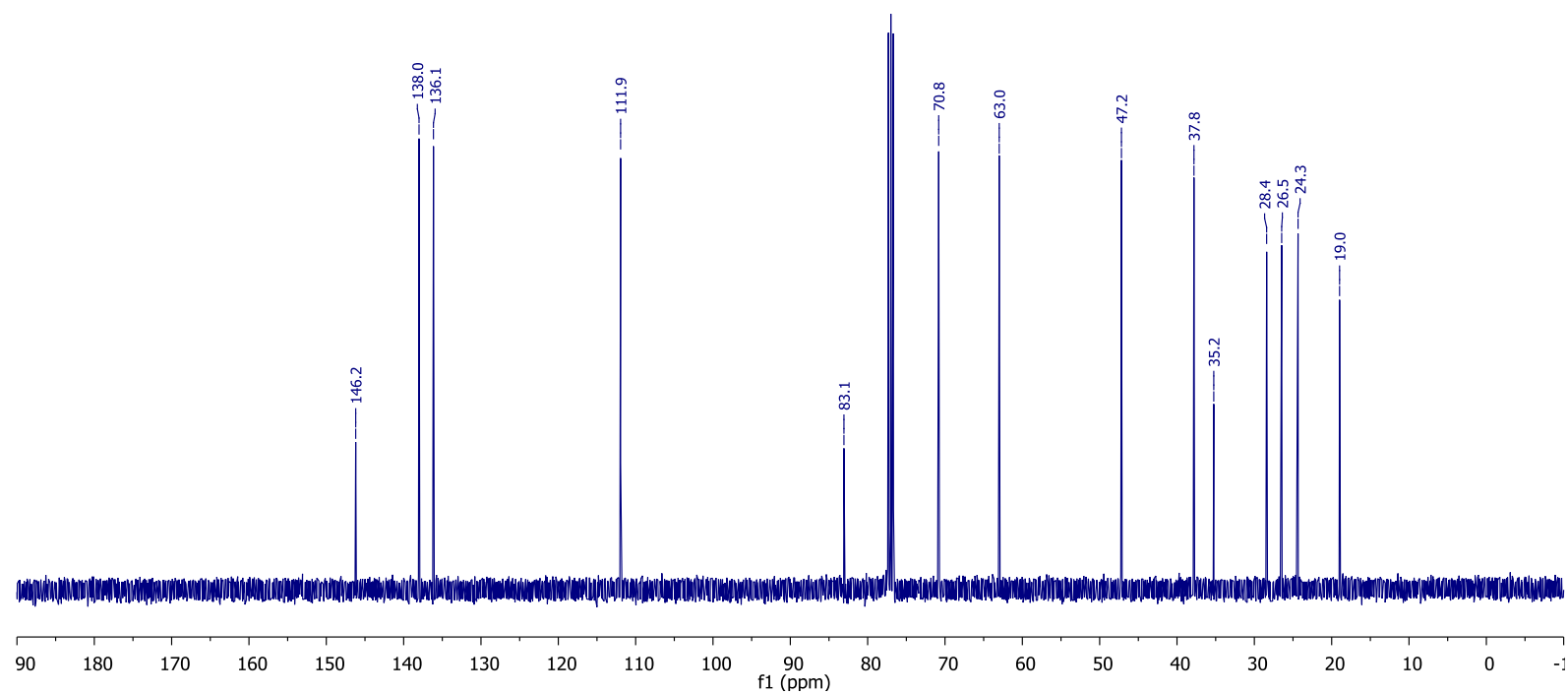


${ }^{1} \mathrm{H}$ NMR (200 MHz, $\mathrm{CDCl}_{3}$ ) of compound-5

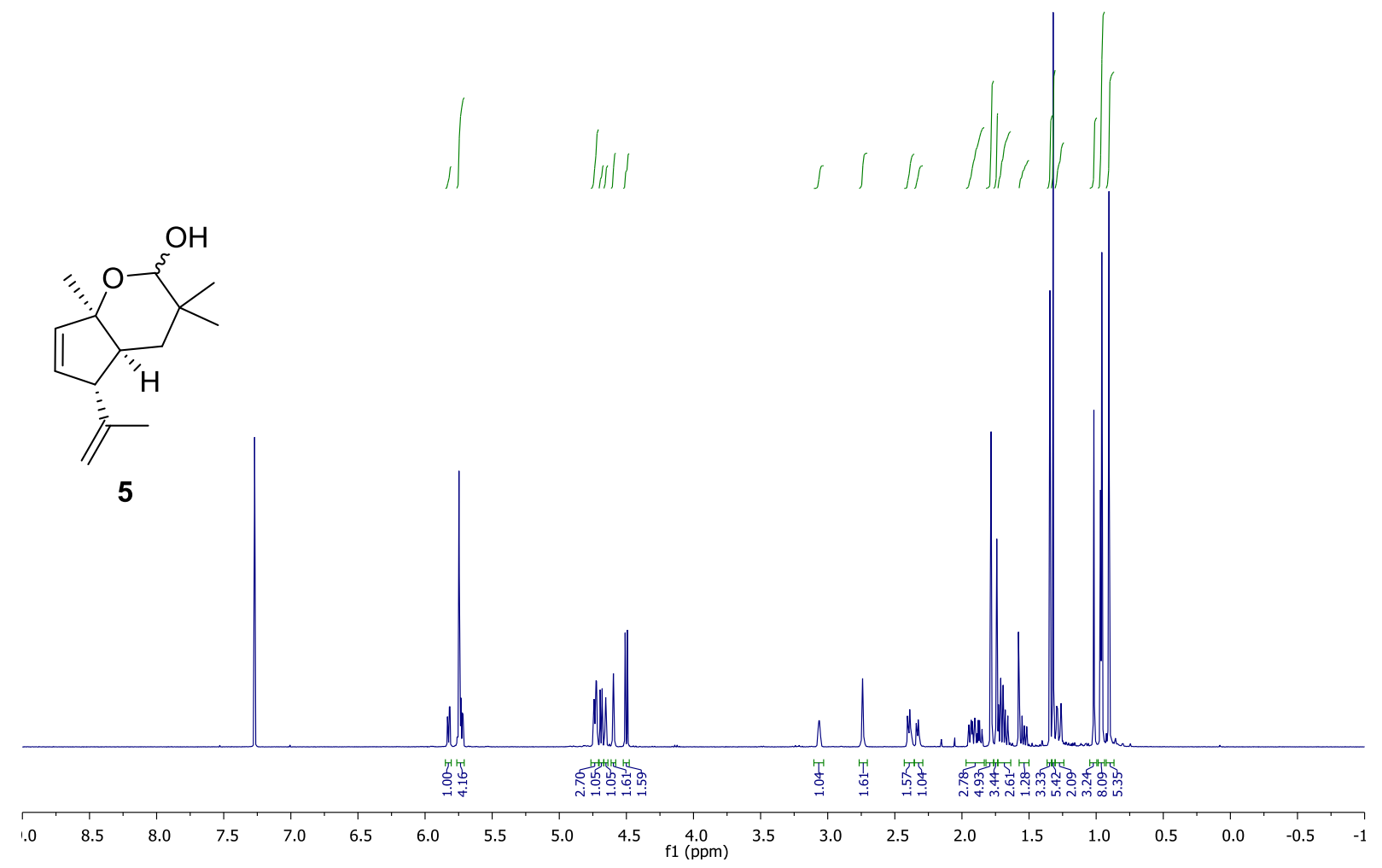

${ }^{13} \mathrm{C}$ NMR (101 MHz, $\mathrm{CDCl}_{3}$ ) of compound-5

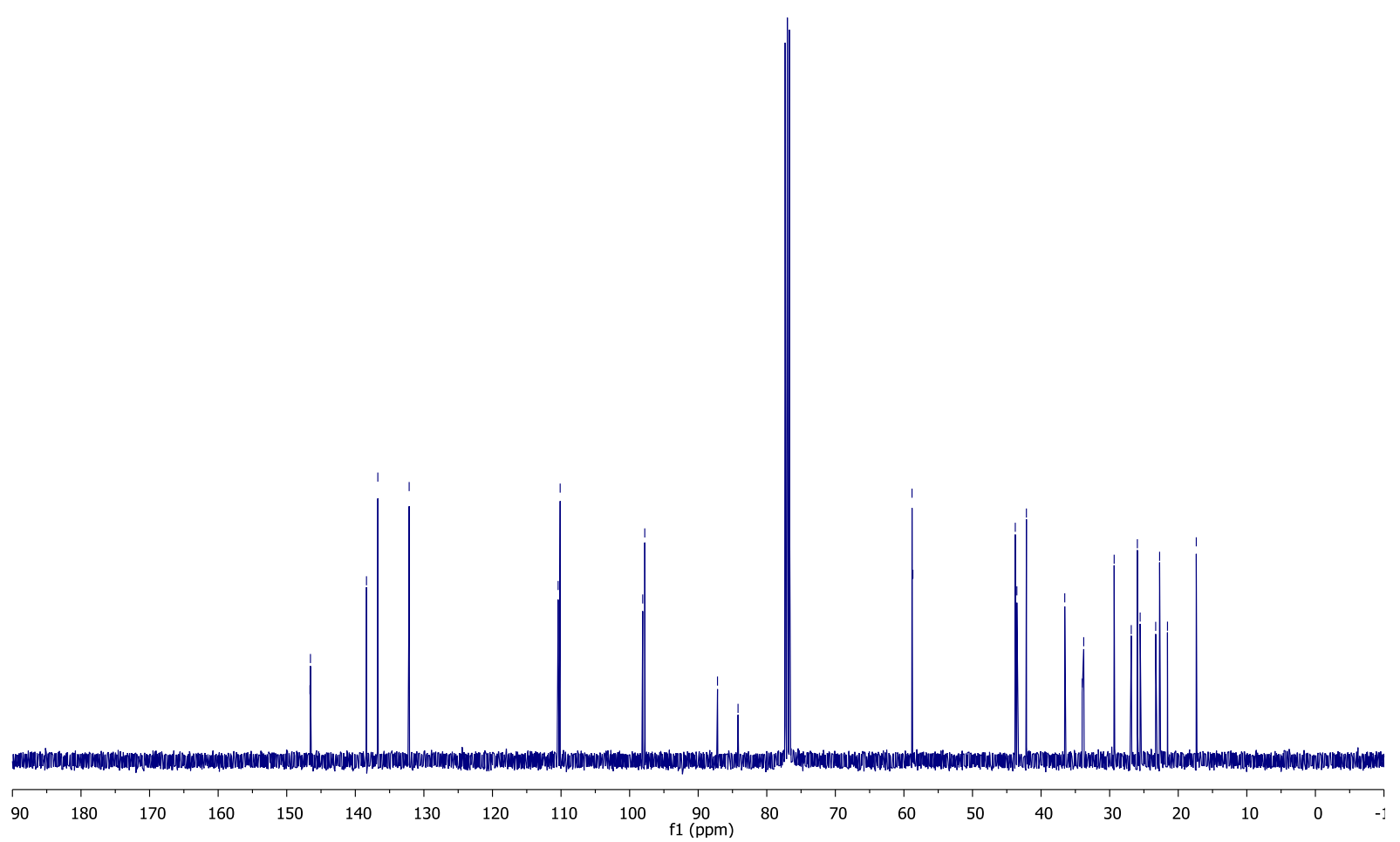


${ }^{1} \mathrm{H}$ NMR (400 MHz, $\mathrm{CDCl}_{3}$ ) of compound-16

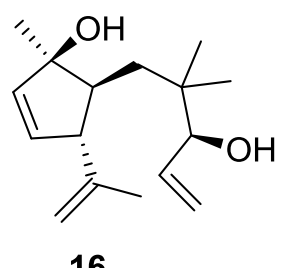

16

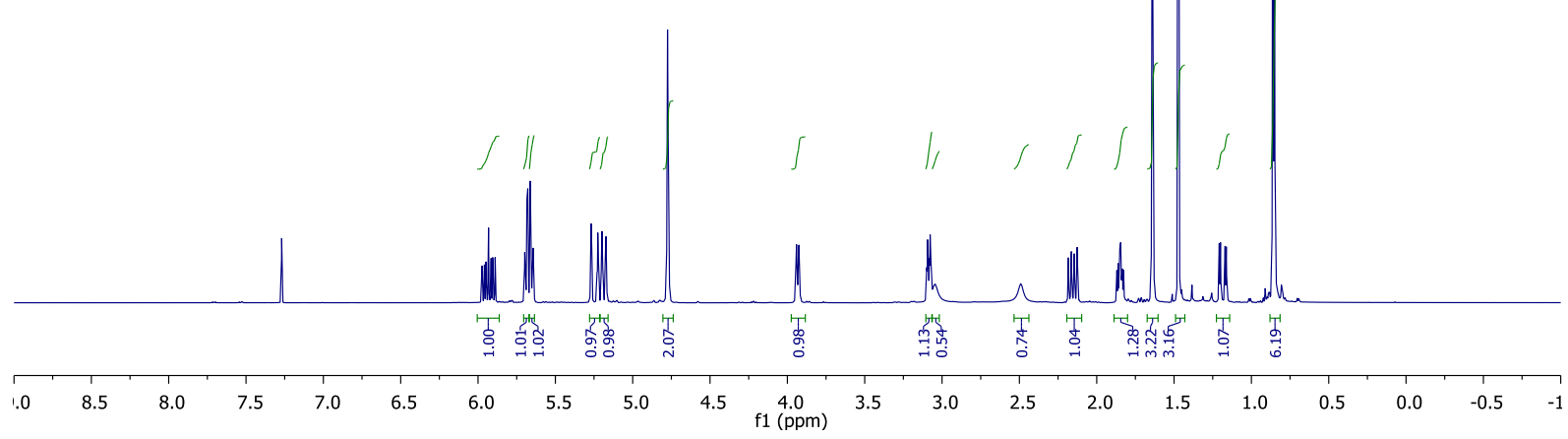

${ }^{13} \mathrm{C}$ NMR (101 MHz, $\mathrm{CDCl}_{3}$ ) of compound-16

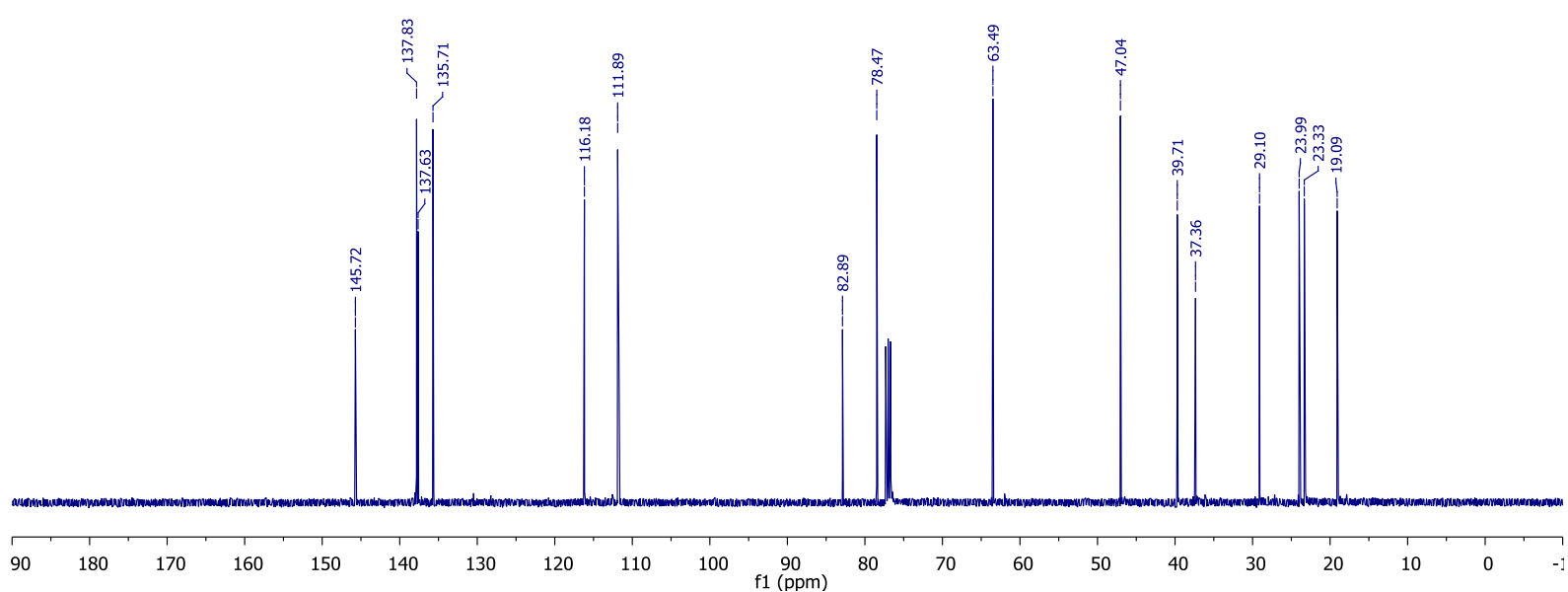


${ }^{1} \mathrm{H}$ NMR (400 MHz, $\mathrm{CDCl}_{3}$ ) of compound-17

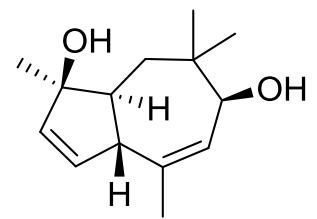

17

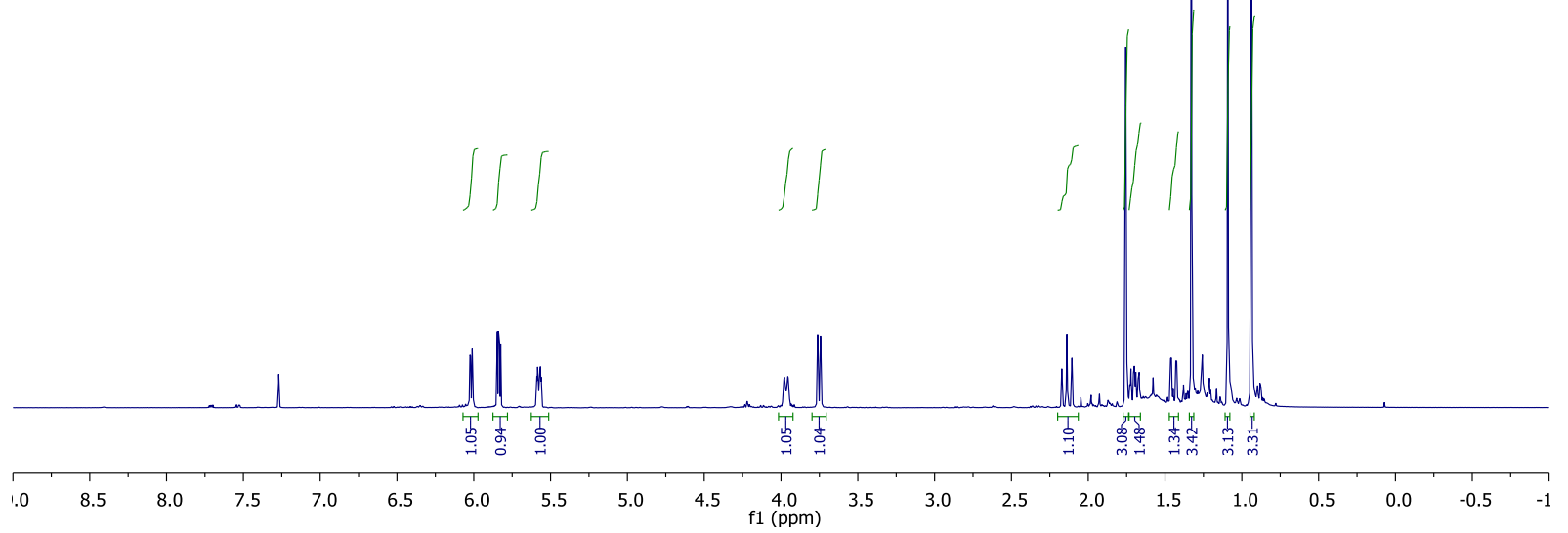

${ }^{13} \mathrm{C}$ NMR (101 MHz, $\mathrm{CDCl}_{3}$ ) of compound-17

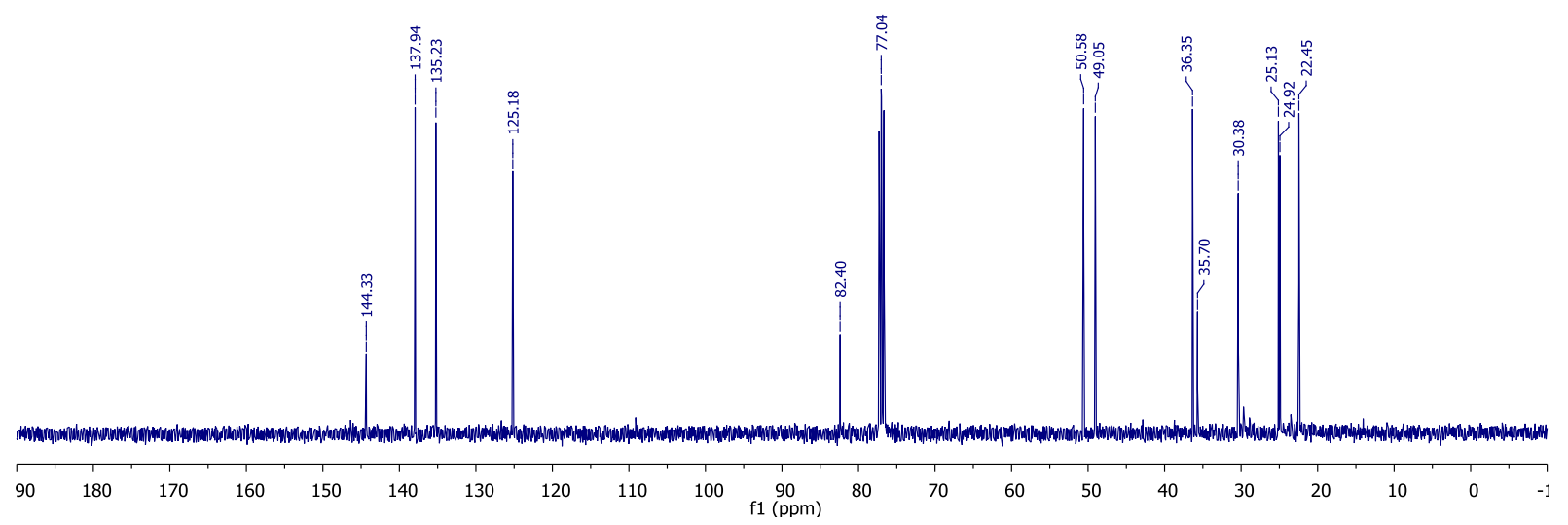


${ }^{1} \mathrm{H}$ NMR (400 MHz, $\mathrm{CDCl}_{3}$ ) of compound-4

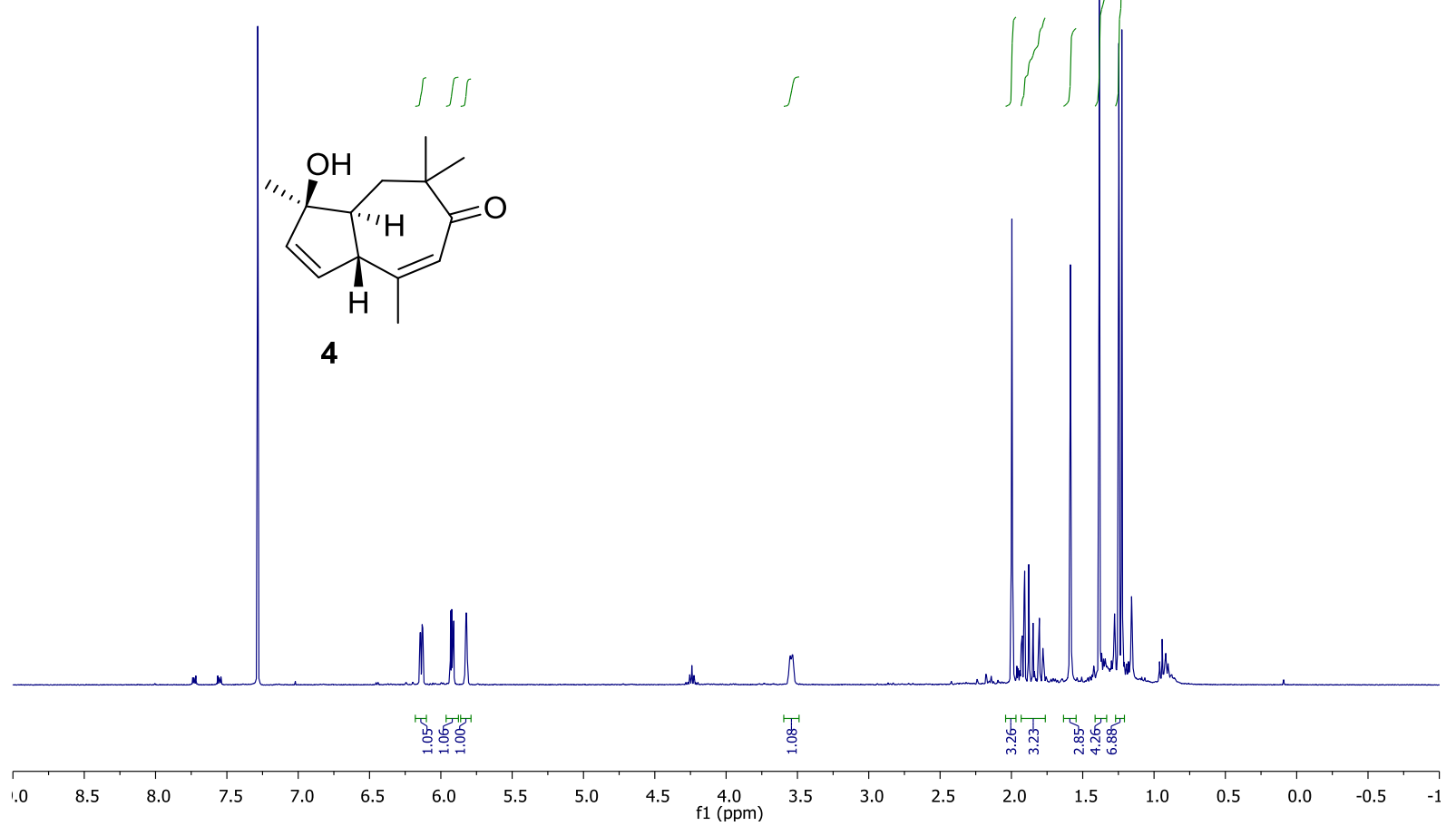

${ }^{13} \mathrm{C}$ NMR (101 MHz, $\mathrm{CDCl}_{3}$ ) of compound-4
।
管

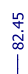

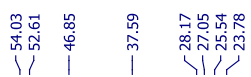

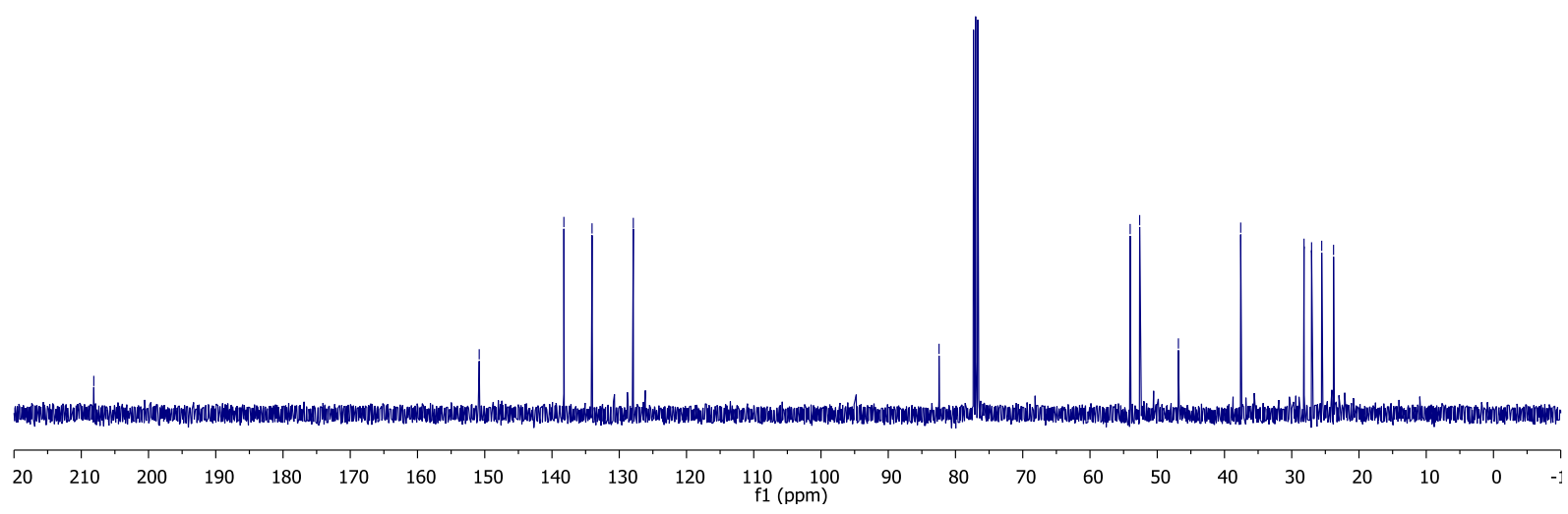


${ }^{1} \mathrm{H}$ NMR (400 MHz, $\mathrm{CDCl}_{3}$ ) of compound-18
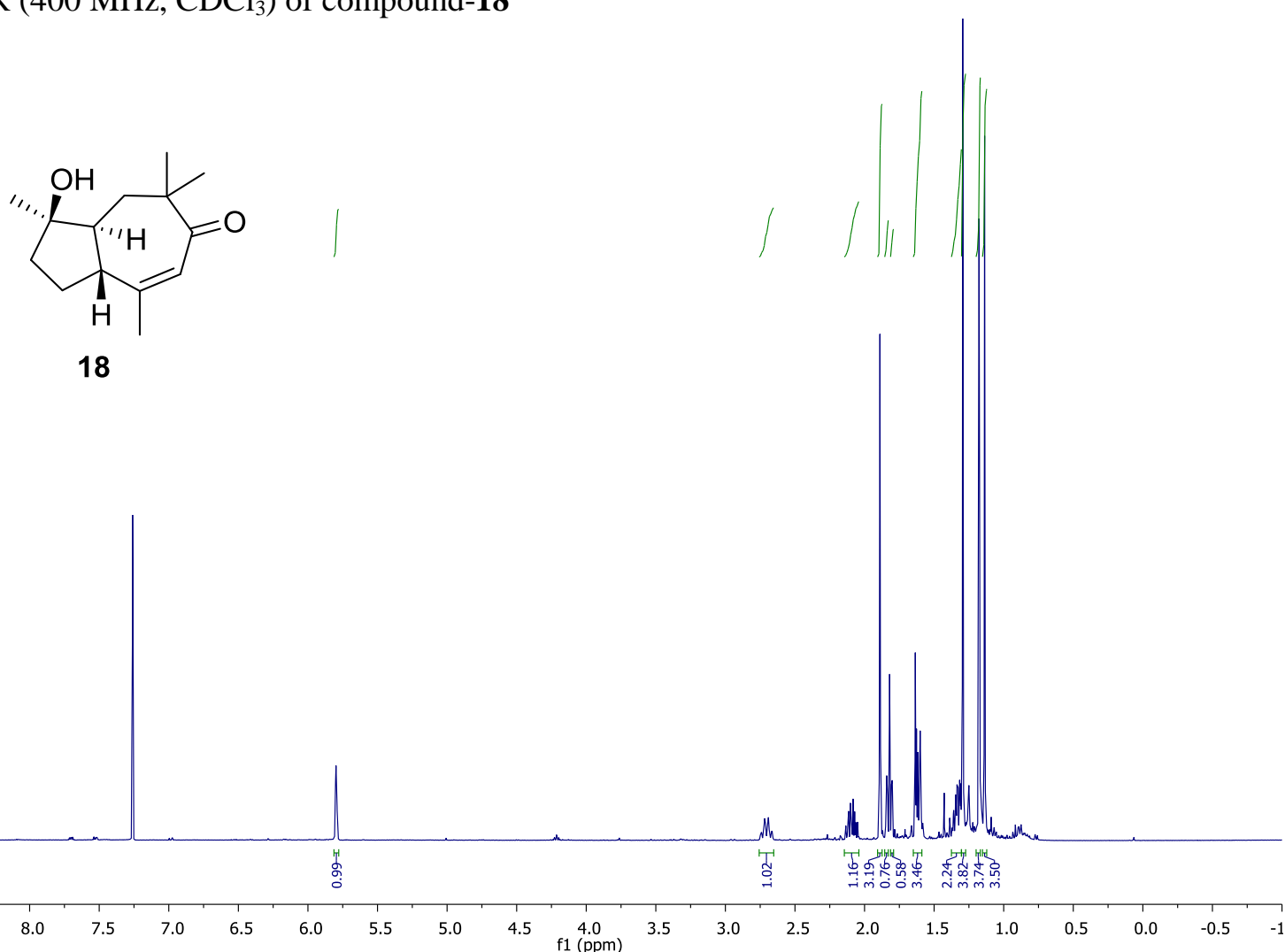

${ }^{13} \mathrm{C}$ NMR (101 MHz, $\mathrm{CDCl}_{3}$ ) of compound-18

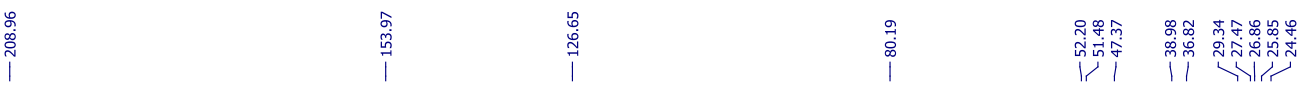

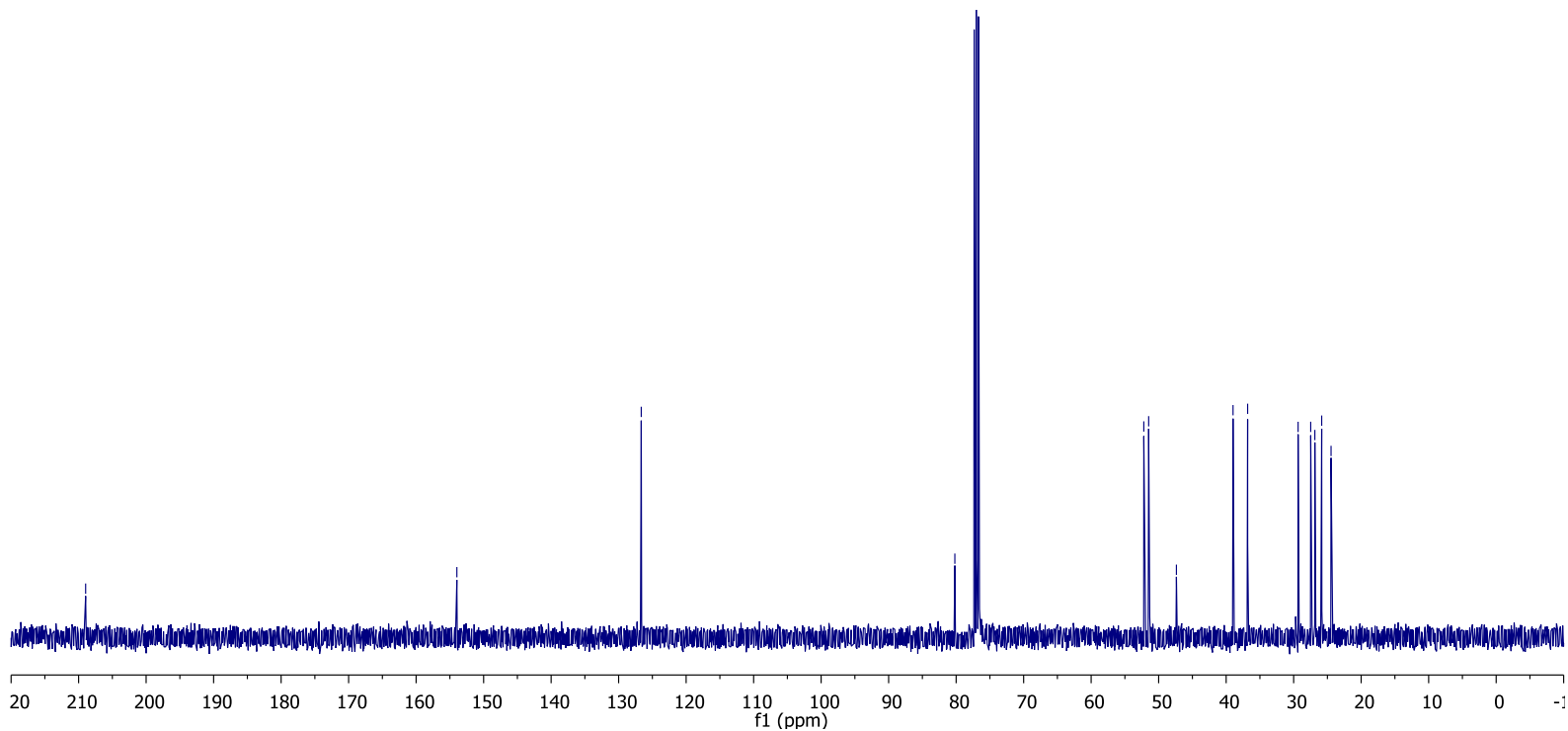


${ }^{1} \mathrm{H}$ NMR (400 MHz, $\mathrm{CDCl}_{3}$ ) of compound-3

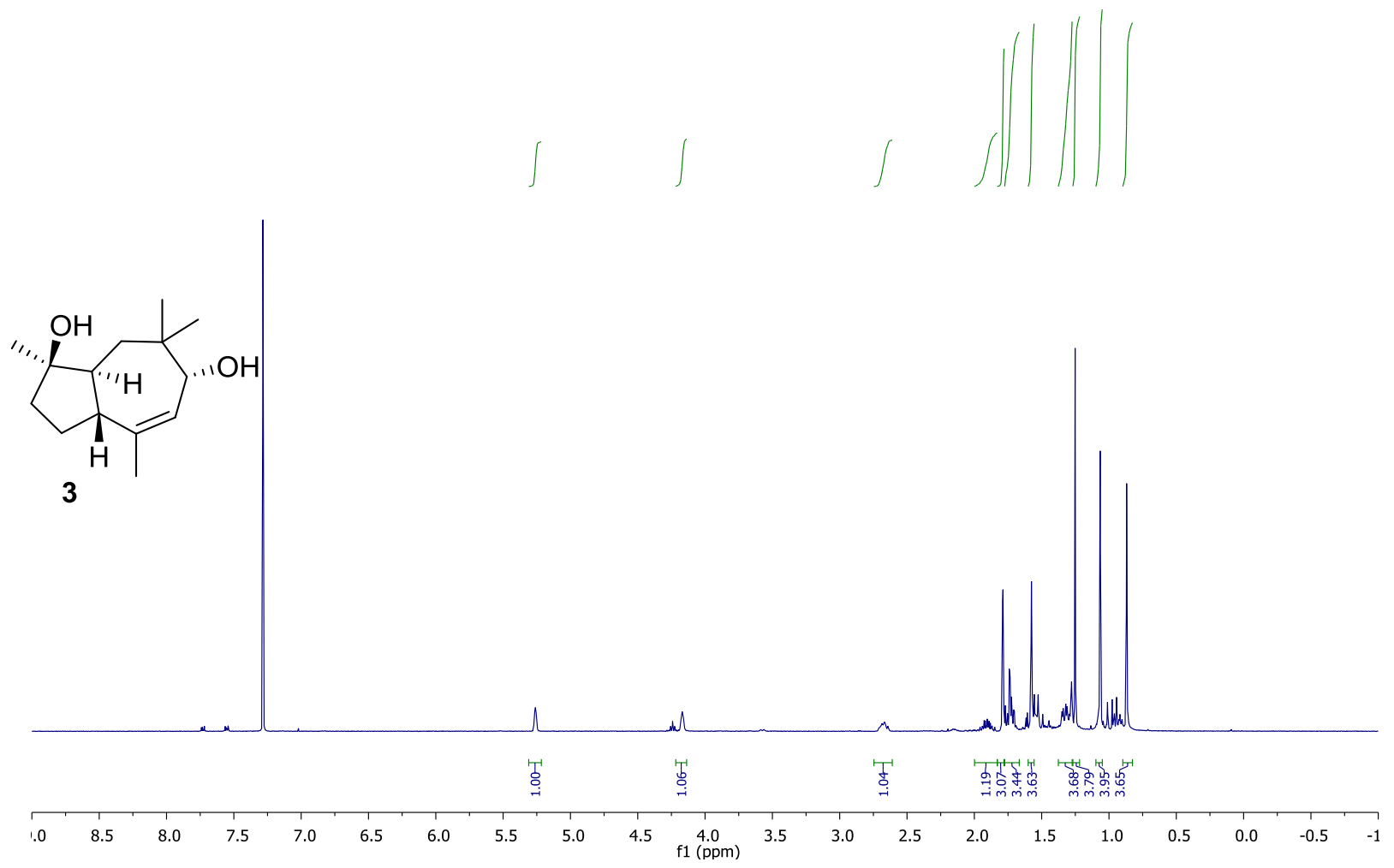

${ }^{13} \mathrm{C}$ NMR (101 MHz, $\mathrm{CDCl}_{3}$ ) of compound-3

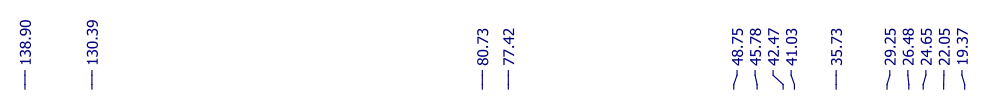

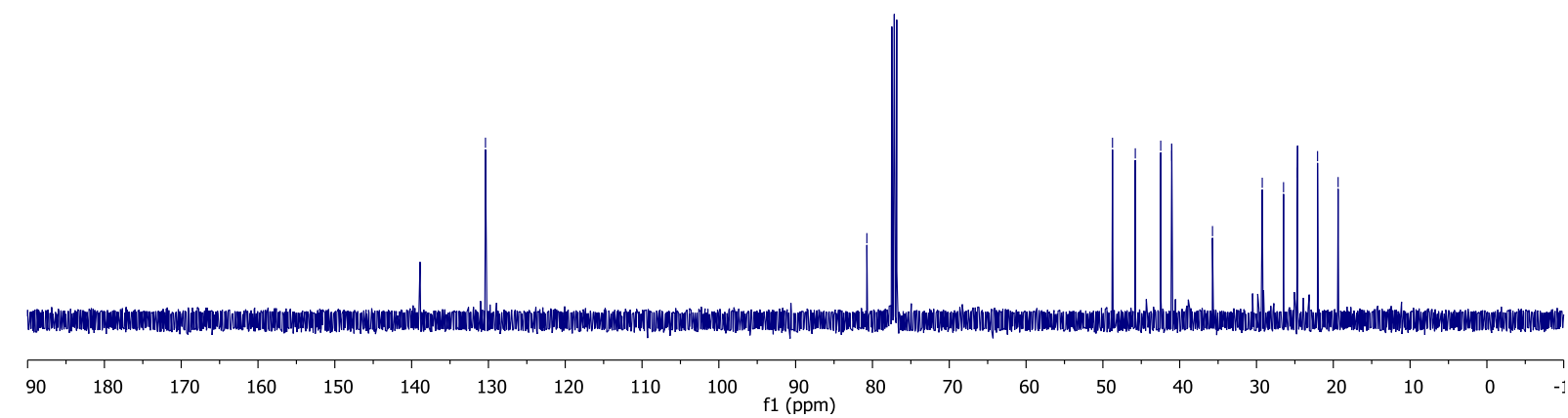


${ }^{1} \mathrm{H}$ NMR (400 MHz, (CD3)2CO) of (+)-omphadiol (1)

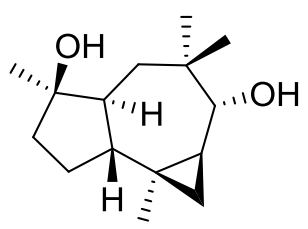

(+)-omphadiol (1)

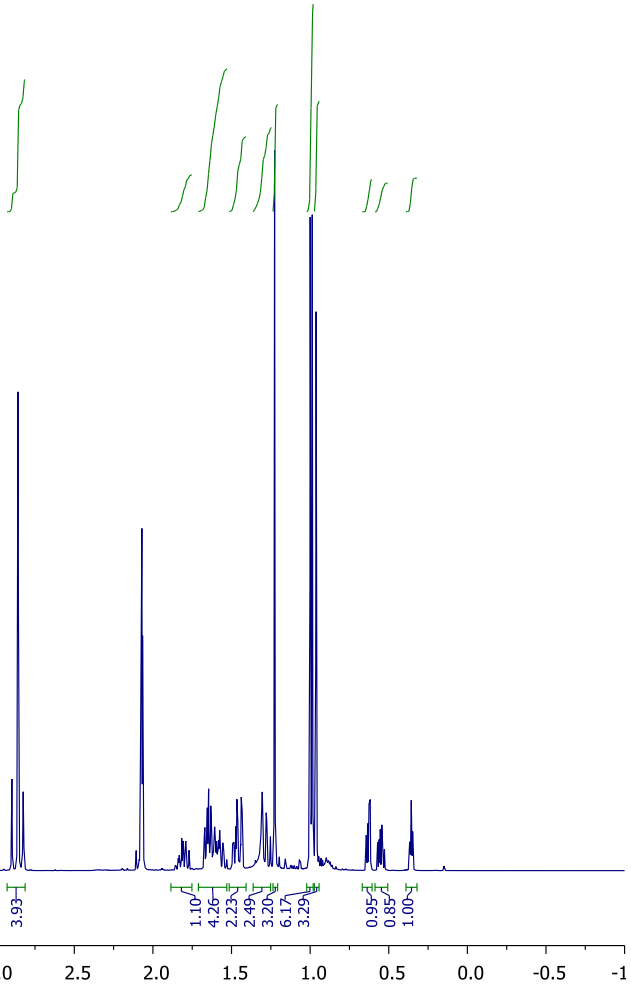

${ }^{13} \mathrm{C}$ NMR (101 MHz, (CD3)2CO) of (+)-omphadiol (1)

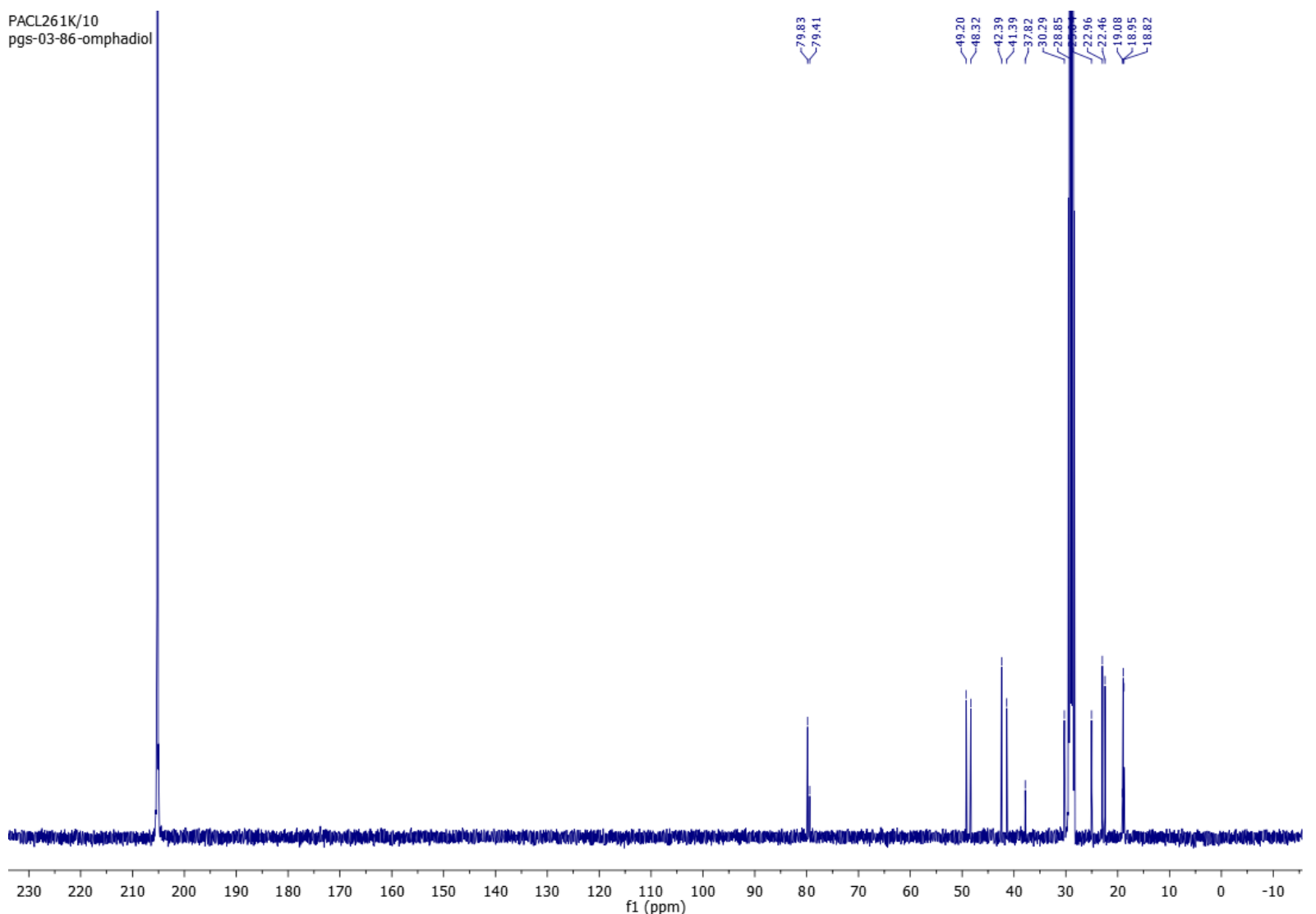


${ }^{1} \mathrm{H}$ NMR $\left(400 \mathrm{MHz}, \mathrm{CDCl}_{3}\right.$ ) of compound $\mathbf{B}$

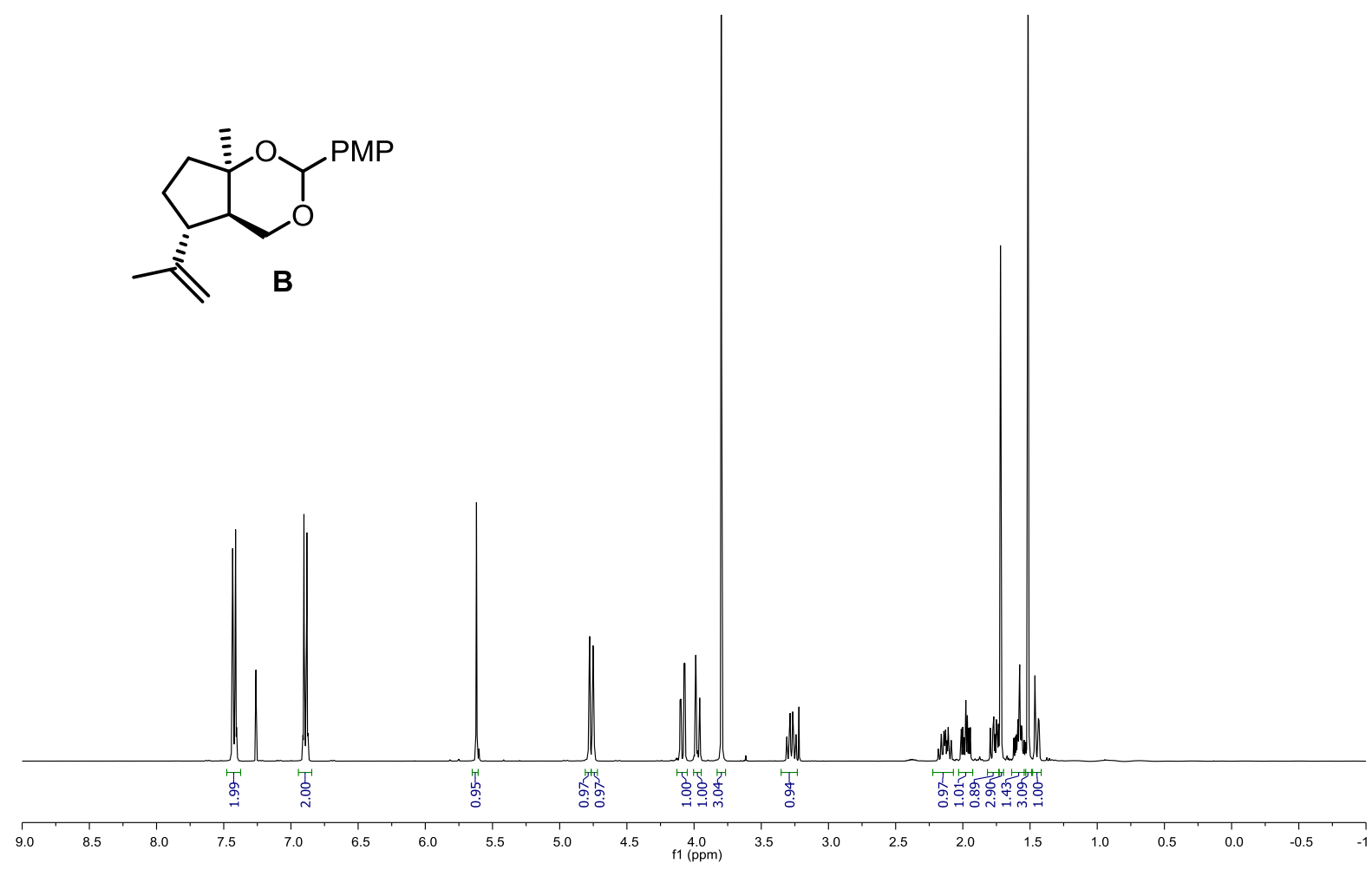

APT-NMR (101 MHz, $\mathrm{CDCl}_{3}$ ) of Compound $\mathbf{B}$

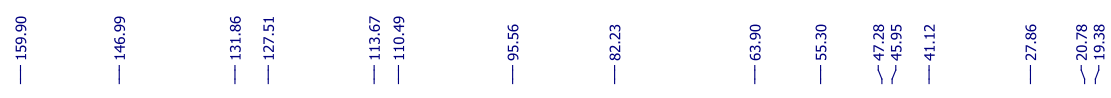

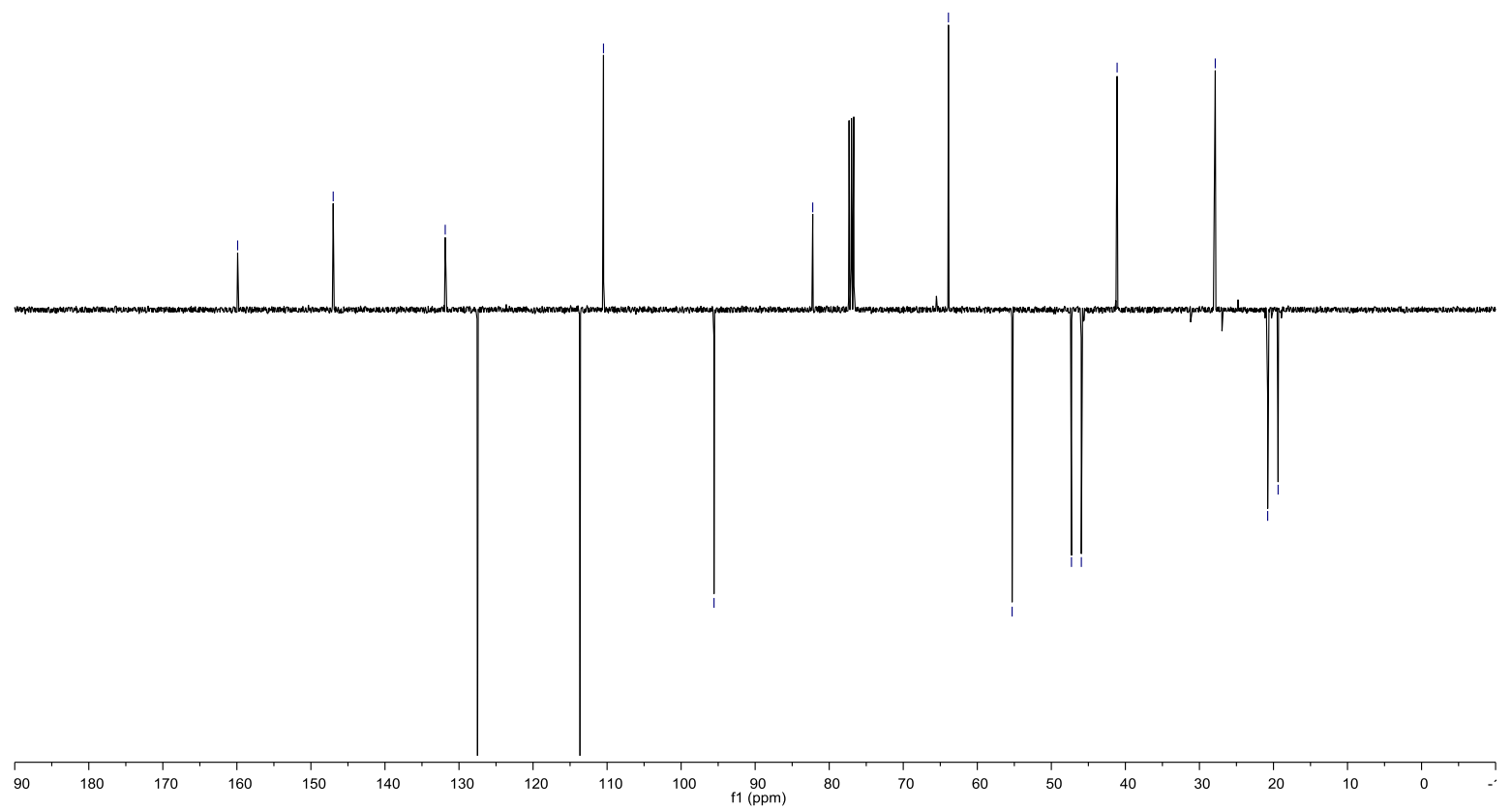




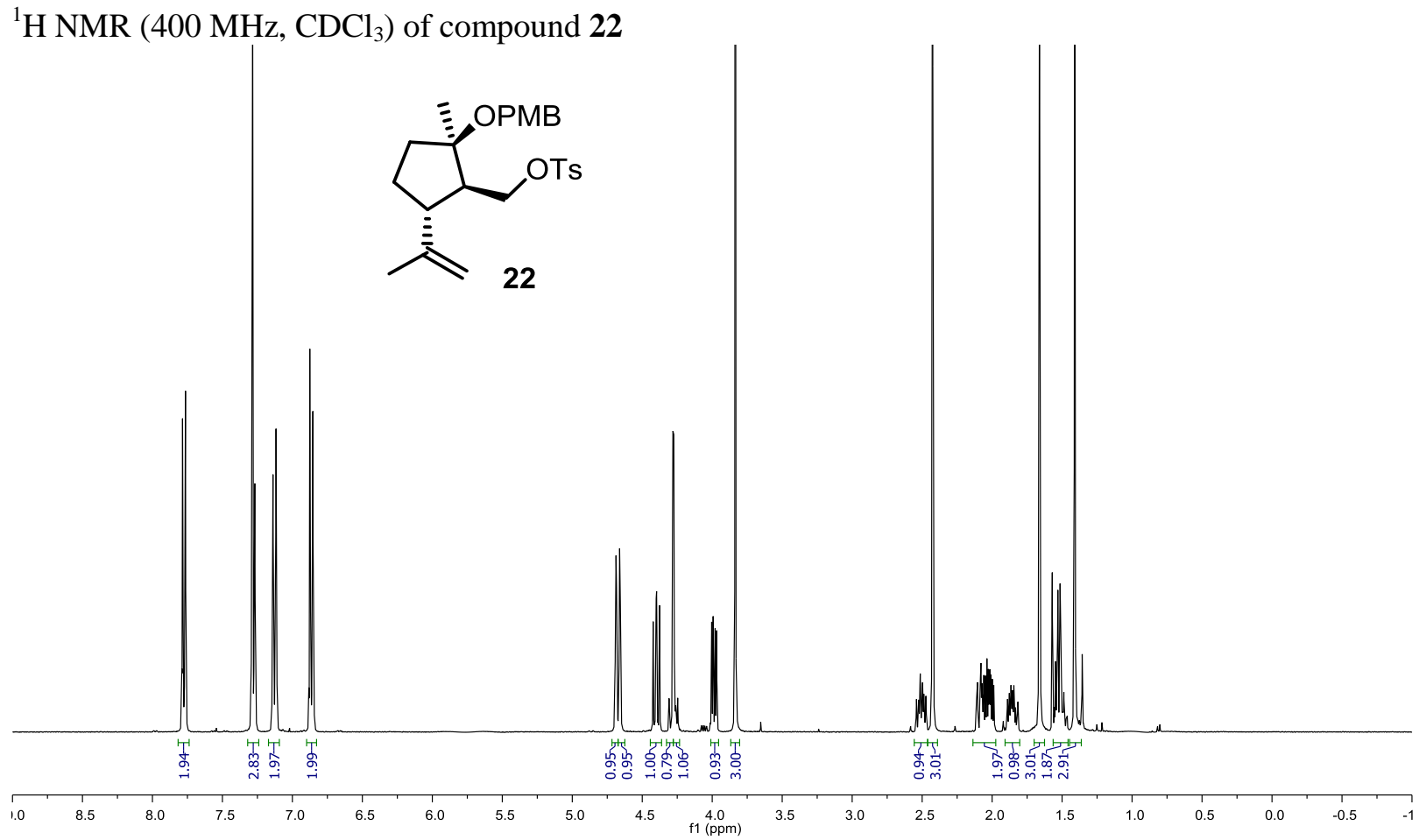

APT-NMR (101 MHz, $\mathrm{CDCl}_{3}$ ) of Compound 22
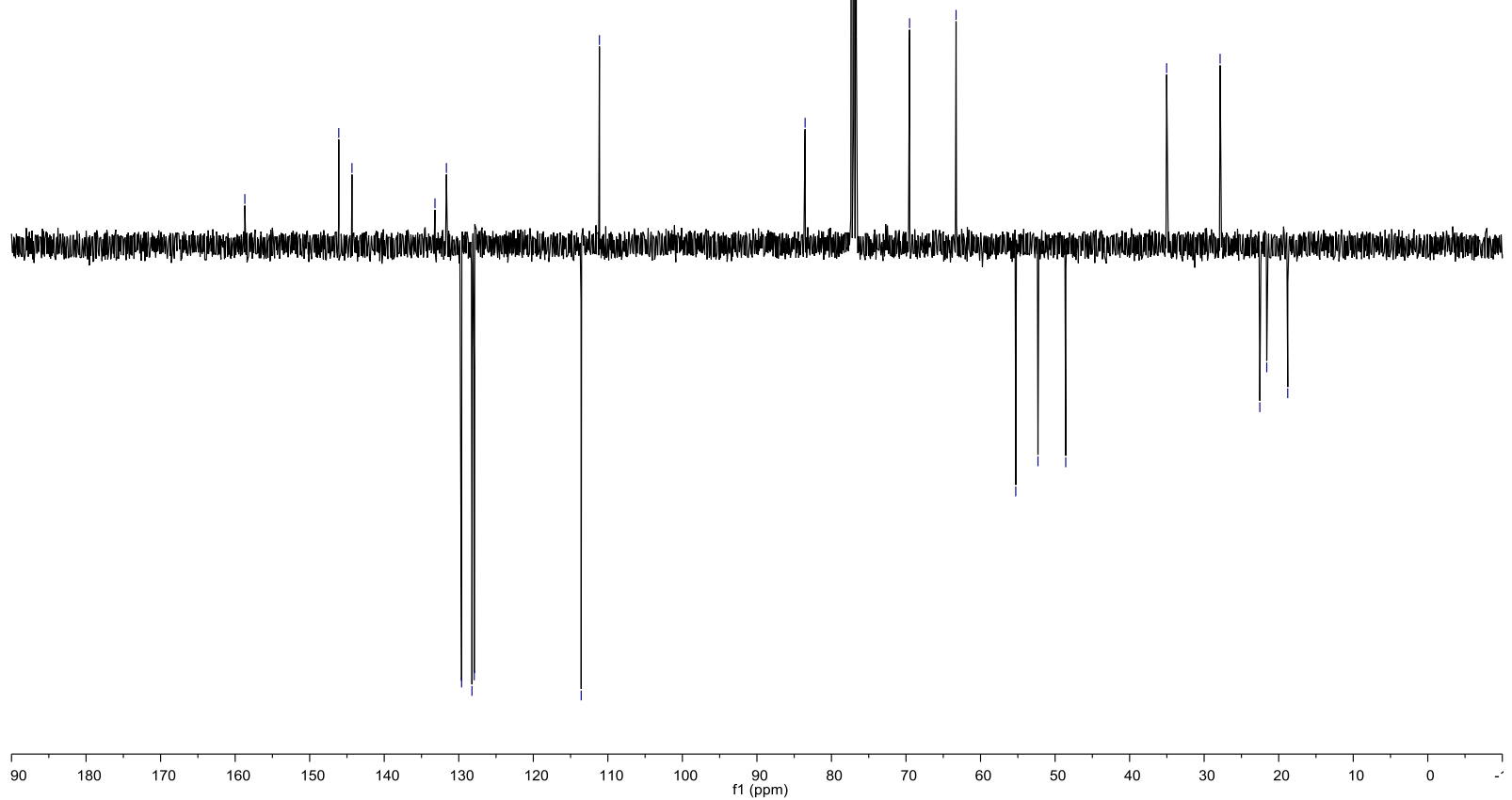
${ }^{1} \mathrm{H}$ NMR (400 MHz, $\mathrm{CDCl}_{3}$ ) of compound $\mathbf{C}$

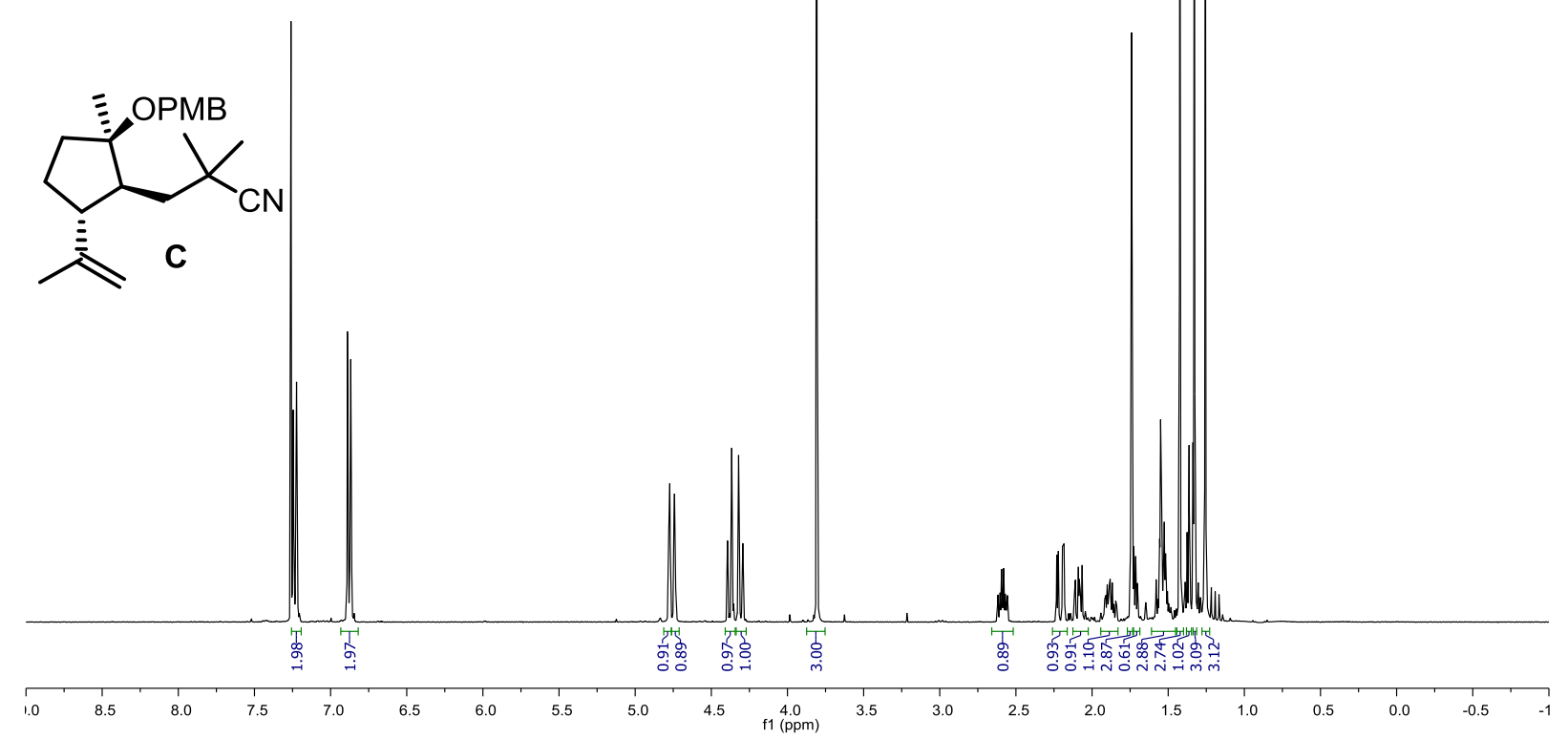

APT-NMR (101 MHz, $\mathrm{CDCl}_{3}$ ) of Compound $\mathbf{C}$

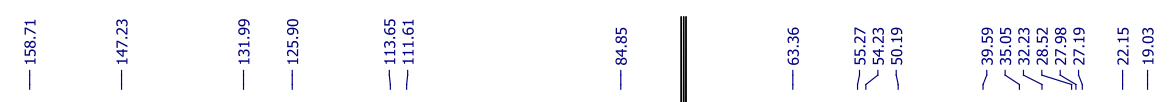

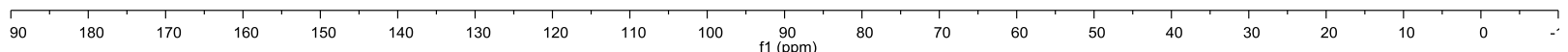


${ }^{1} \mathrm{H}$ NMR (400 MHz, $\mathrm{CDCl}_{3}$ ) of compound 21
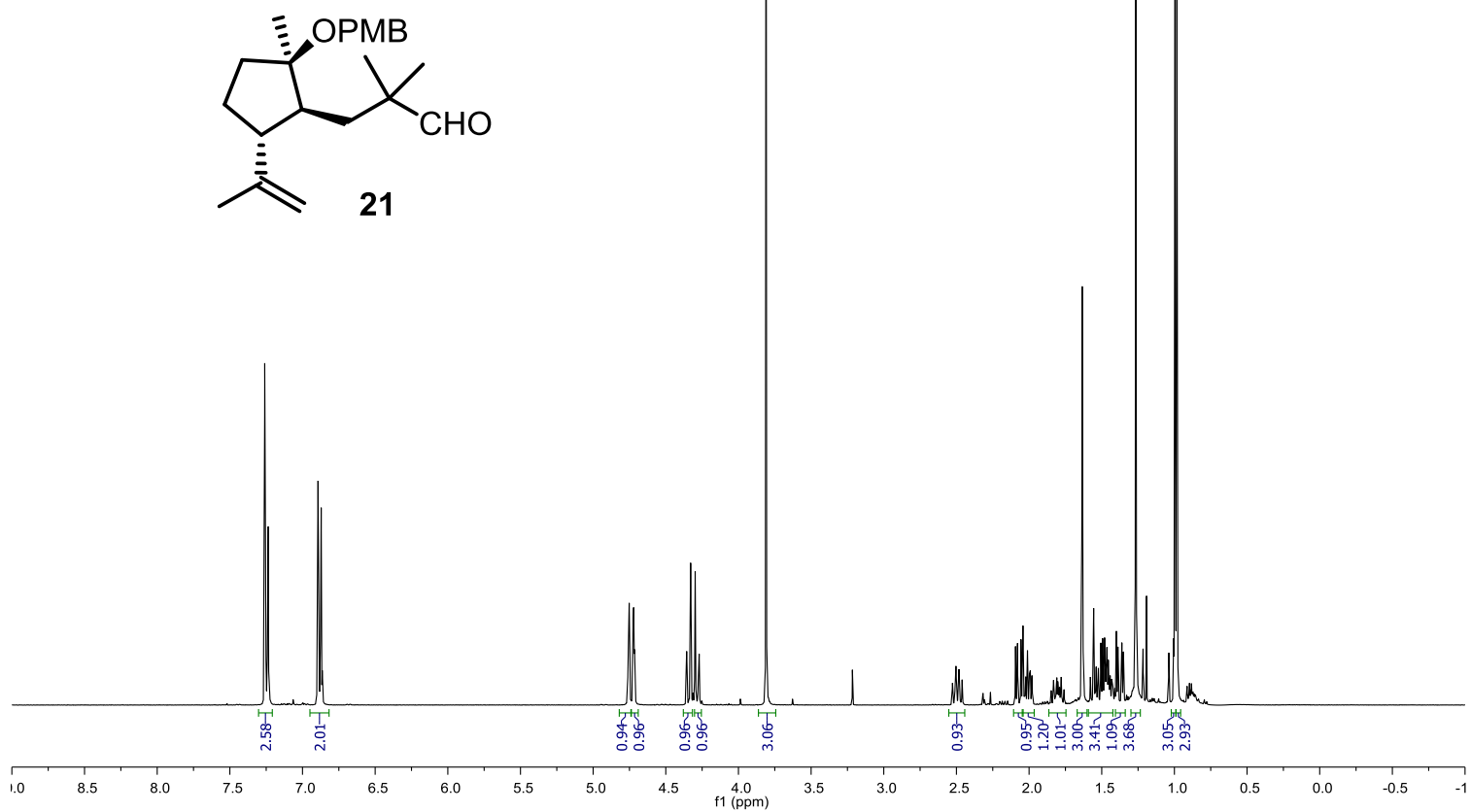

APT-NMR (101 MHz, $\mathrm{CDCl}_{3}$ ) of Compound 21

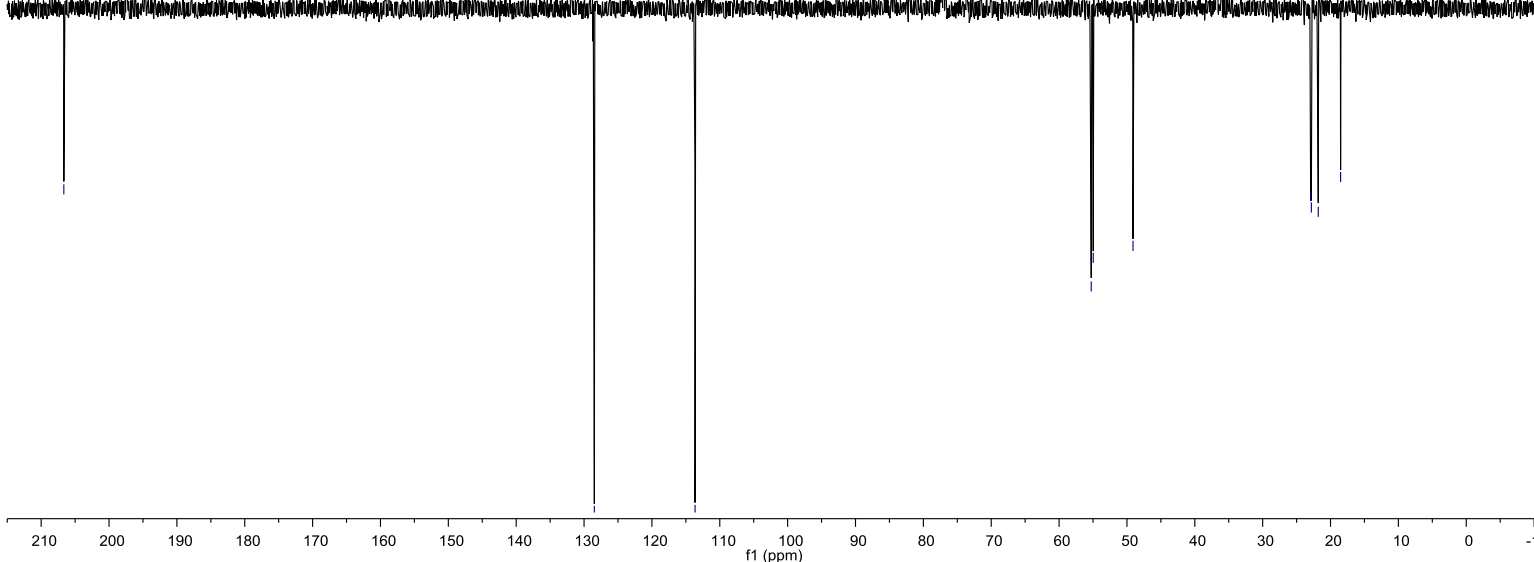


${ }^{1} \mathrm{H}$ NMR (400 MHz, $\mathrm{CDCl}_{3}$ ) of compound 26
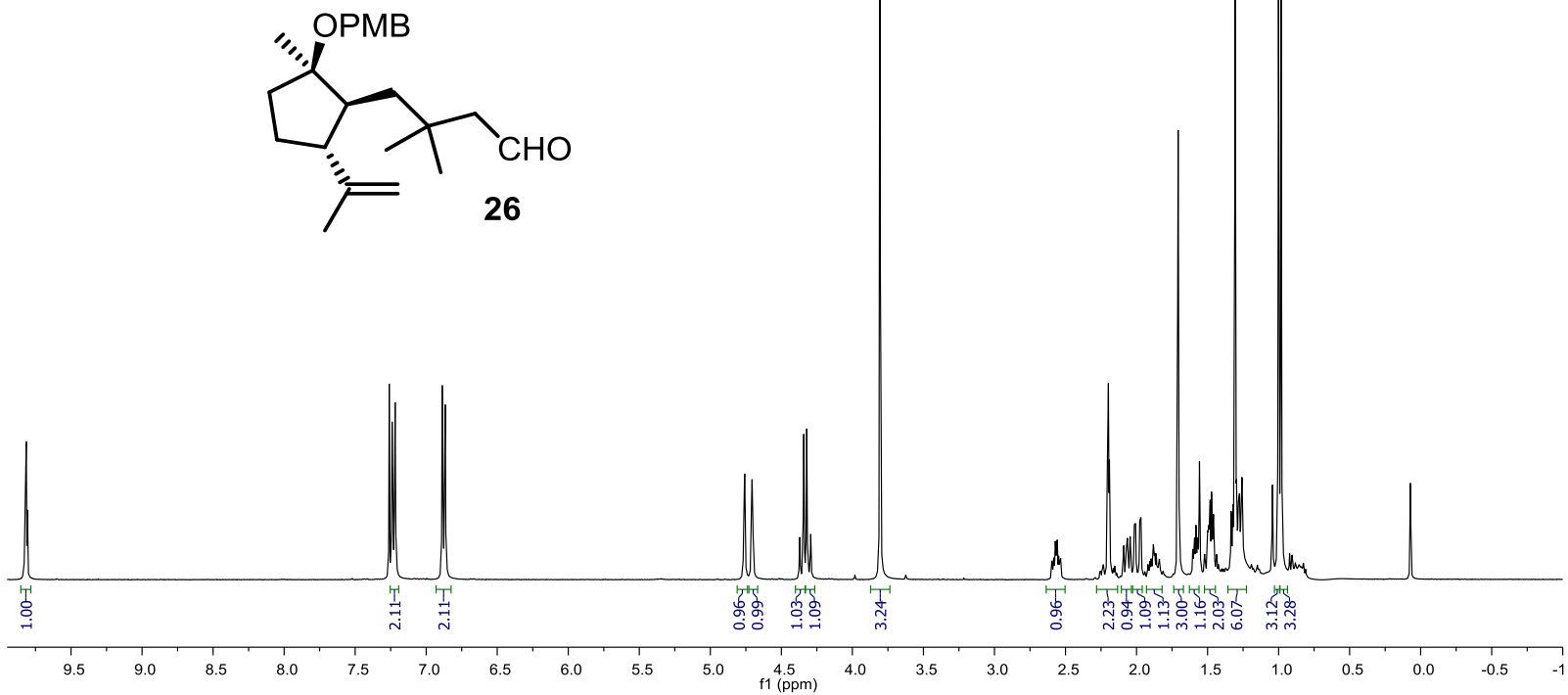

APT-NMR (101 MHz, $\mathrm{CDCl}_{3}$ ) of Compound 26
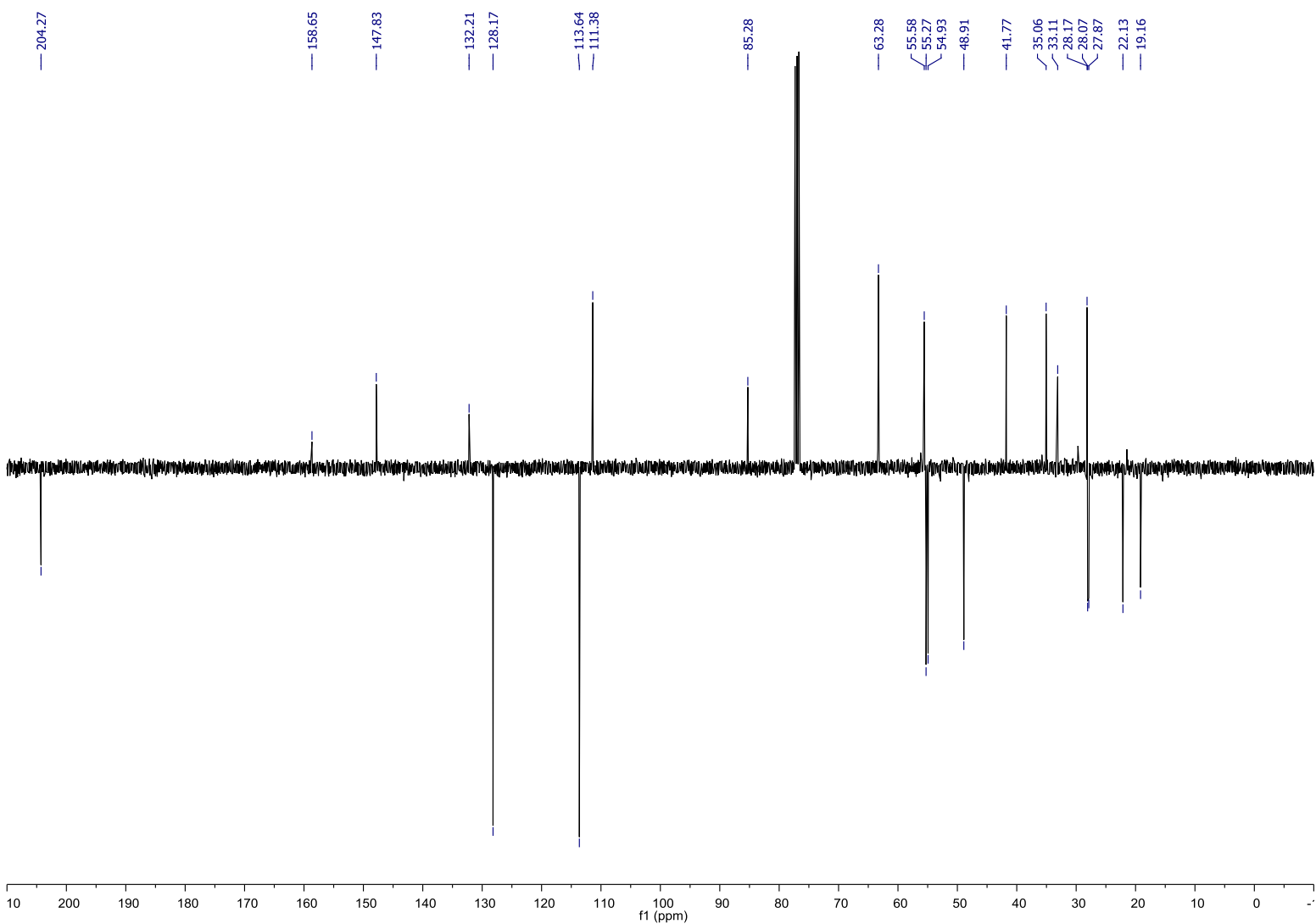
${ }^{1} \mathrm{H}-\mathrm{NMR}\left(400 \mathrm{MHz}, \mathrm{CDCl}_{3}\right.$ ) of compound 20

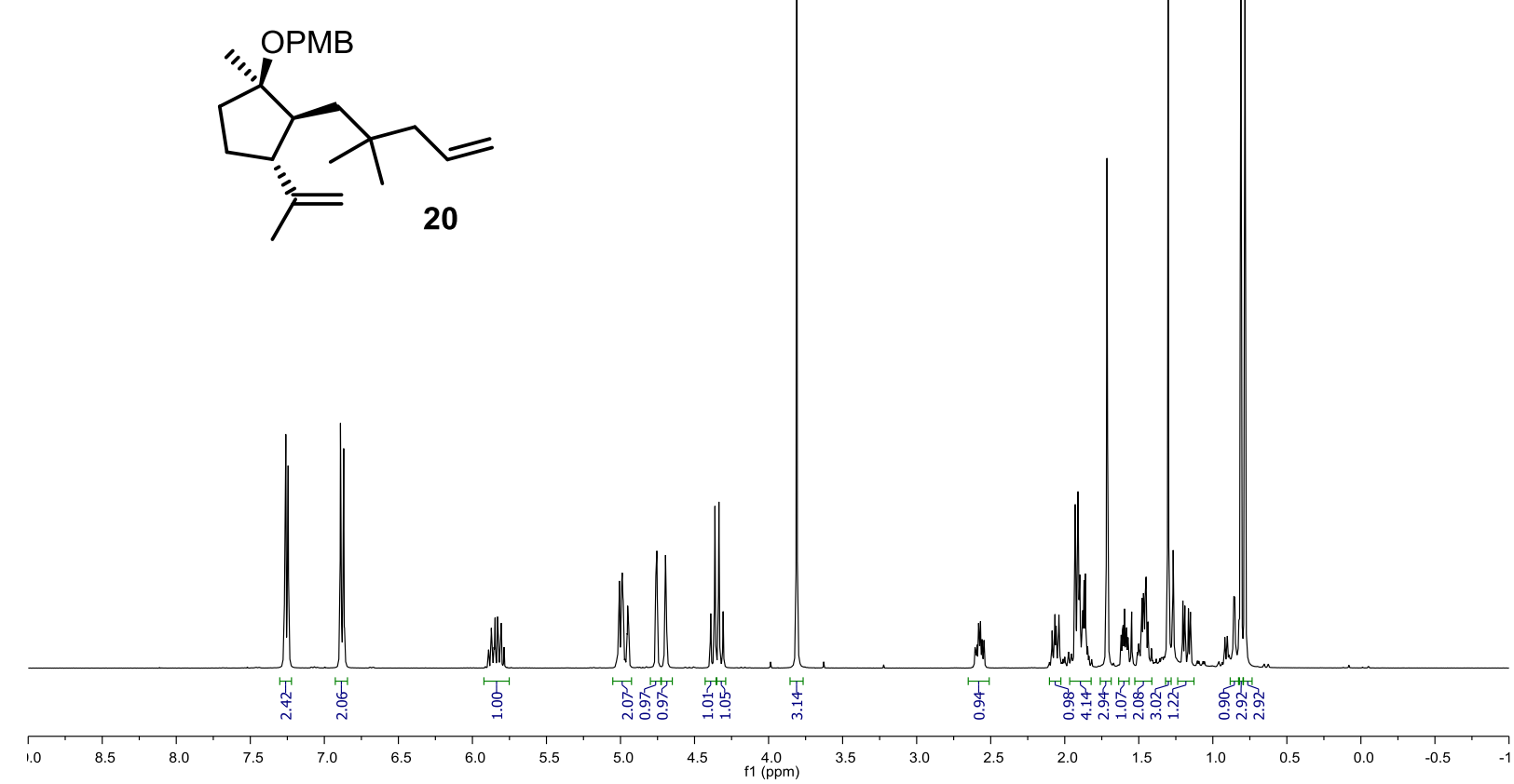

APT-NMR (101 MHz, $\mathrm{CDCl}_{3}$ ) of Compound 20

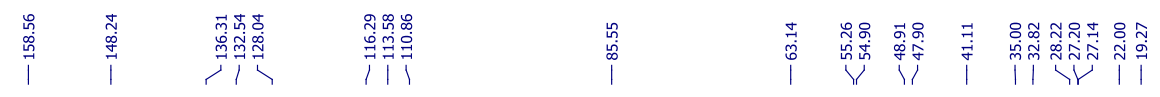

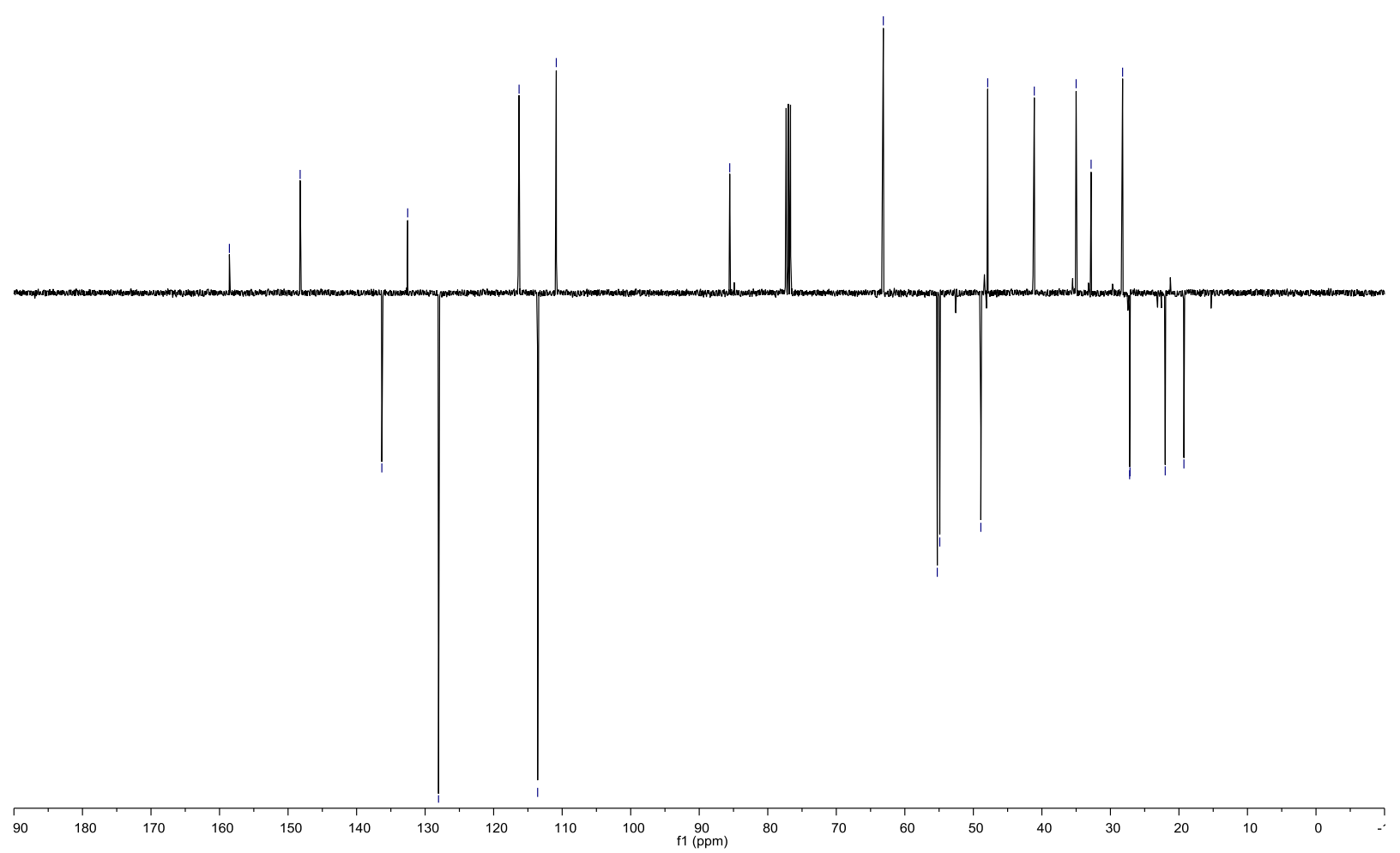


${ }^{1} \mathrm{H}$ NMR (400 MHz, $\mathrm{CDCl}_{3}$ ) of compound 27
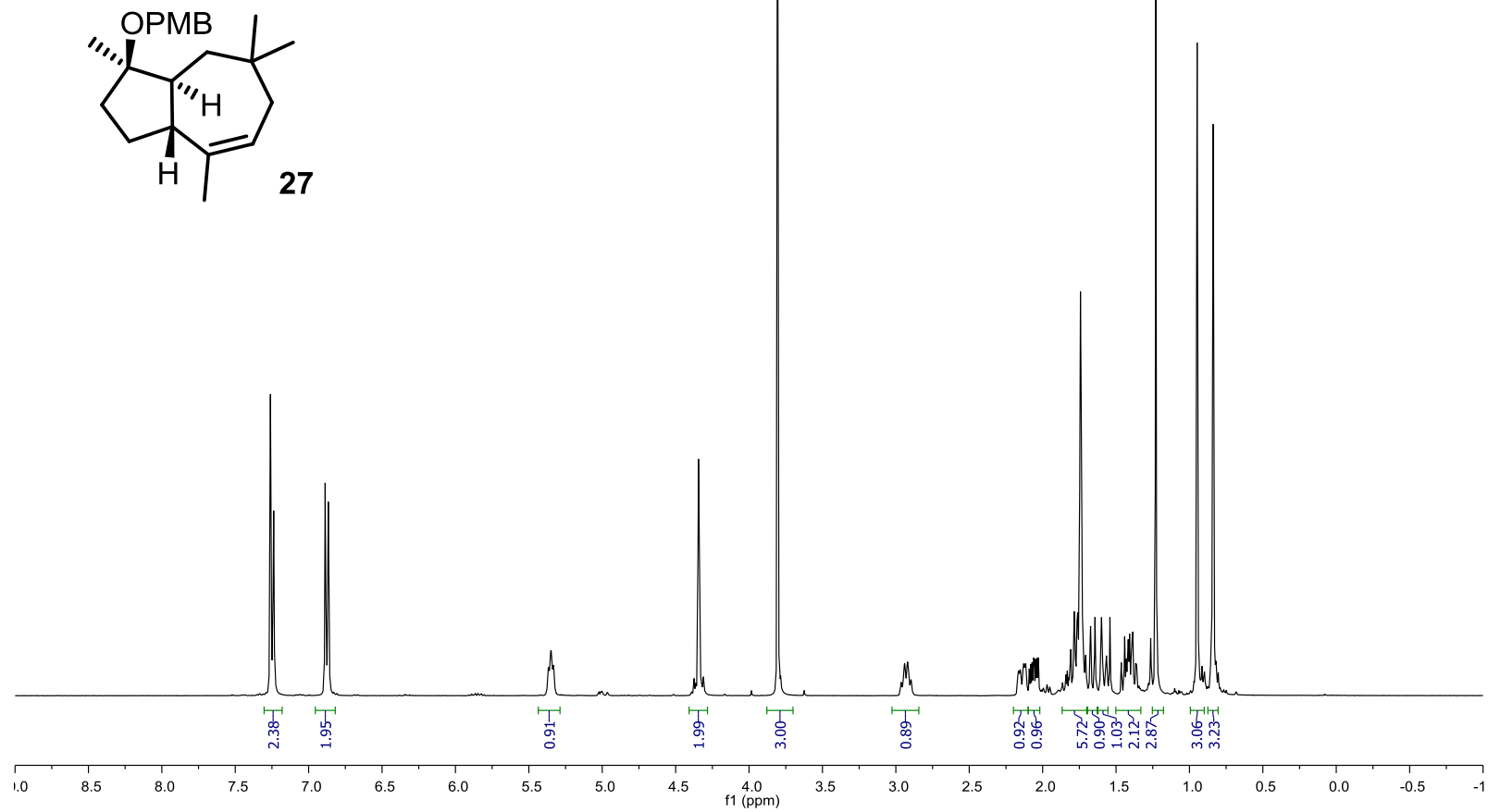

APT-NMR (101 MHz, $\mathrm{CDCl}_{3}$ ) of Compound 27

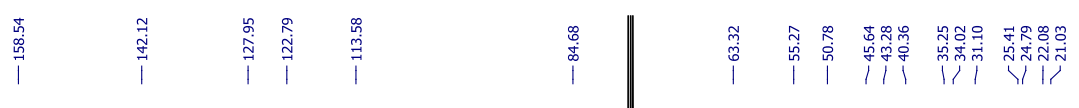

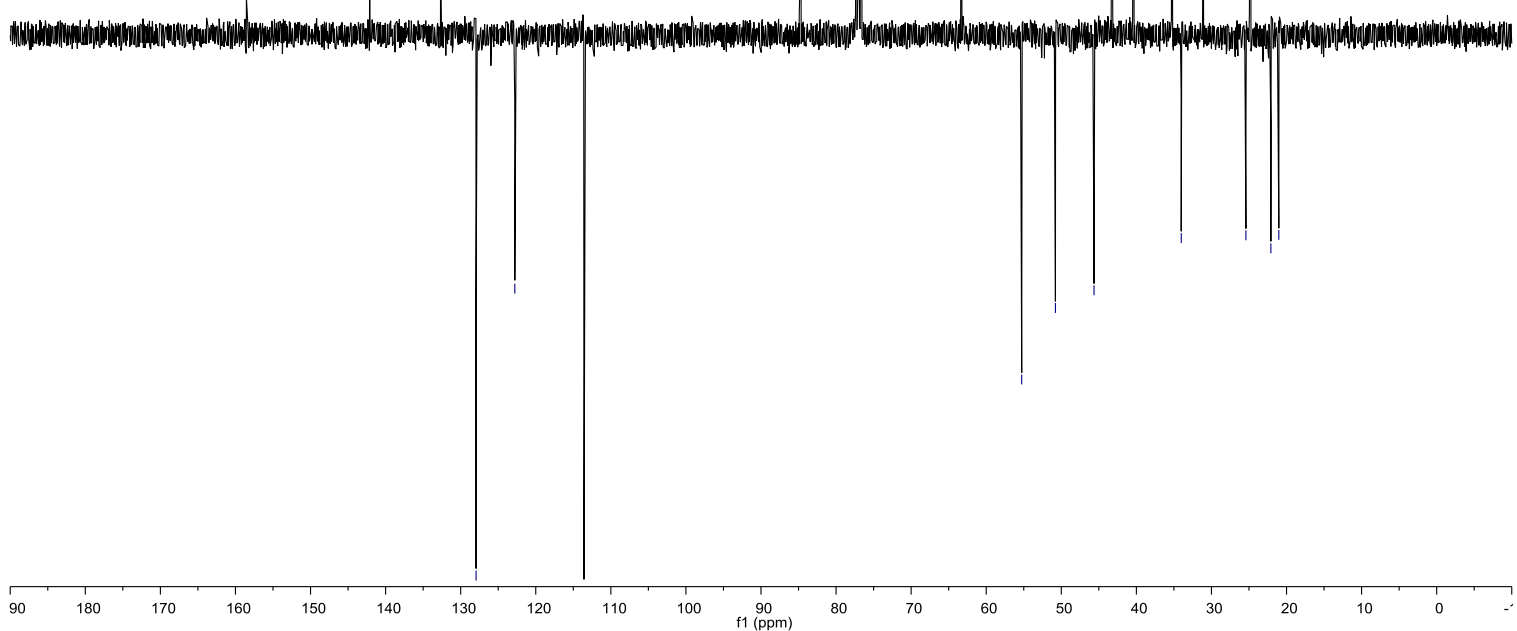


${ }^{1} \mathrm{H}$ NMR (400 MHz, $\mathrm{CDCl}_{3}$ ) of compound 19
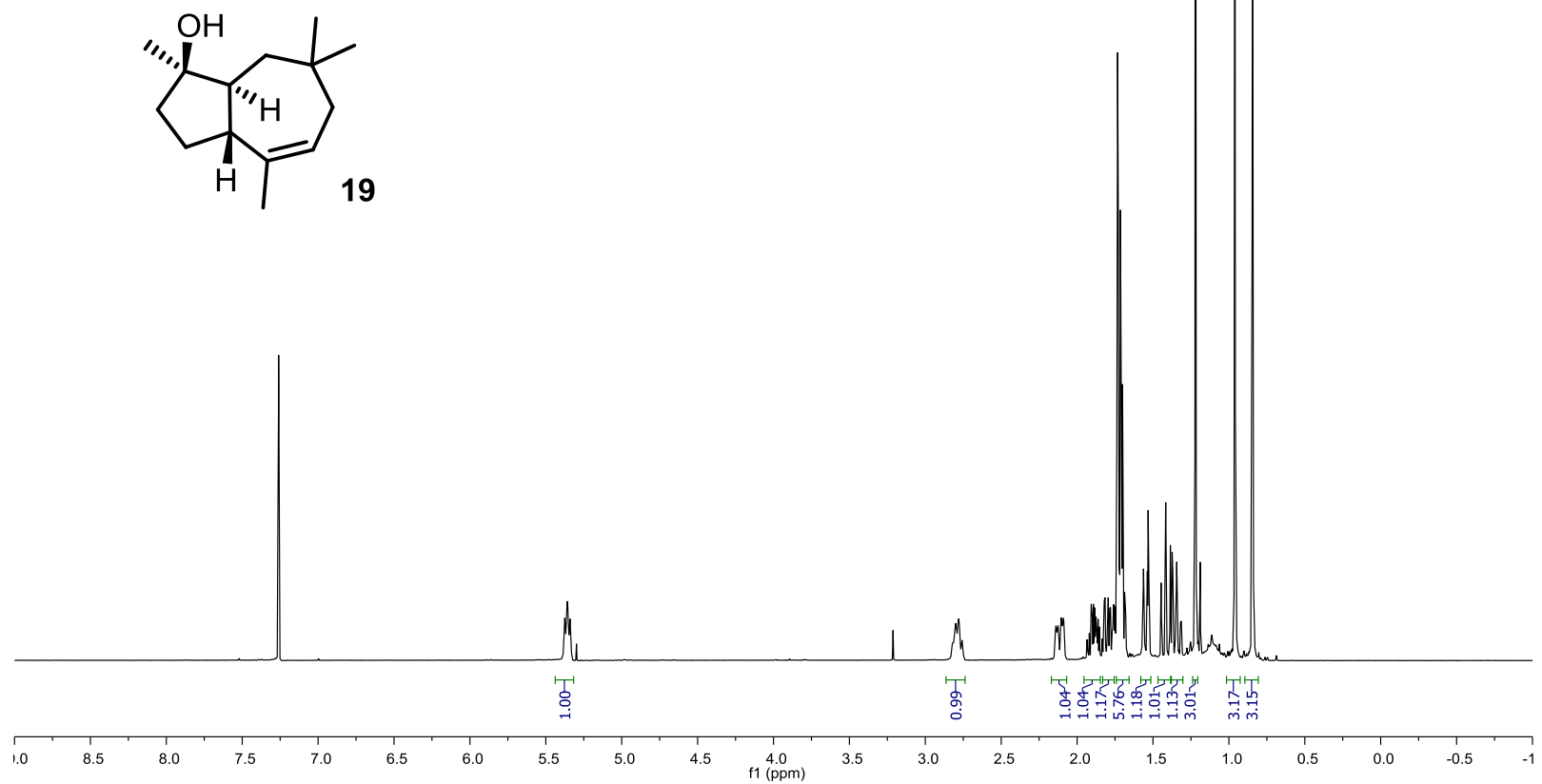

APT-NMR (101 MHz, $\mathrm{CDCl}_{3}$ ) of Compound 19

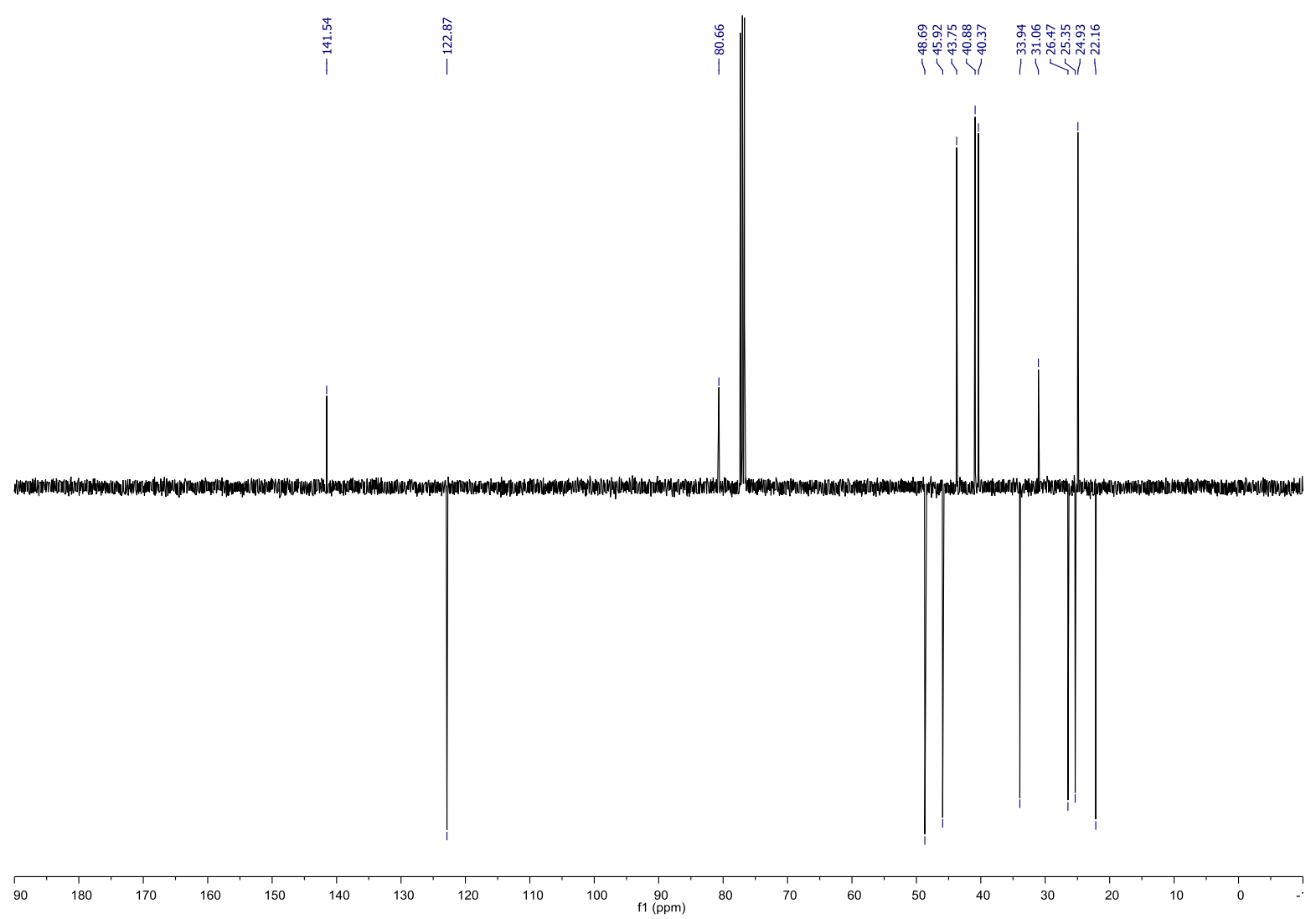


${ }^{1} \mathrm{H}$ NMR (400 MHz, $\mathrm{CDCl}_{3}$ ) of compound 29

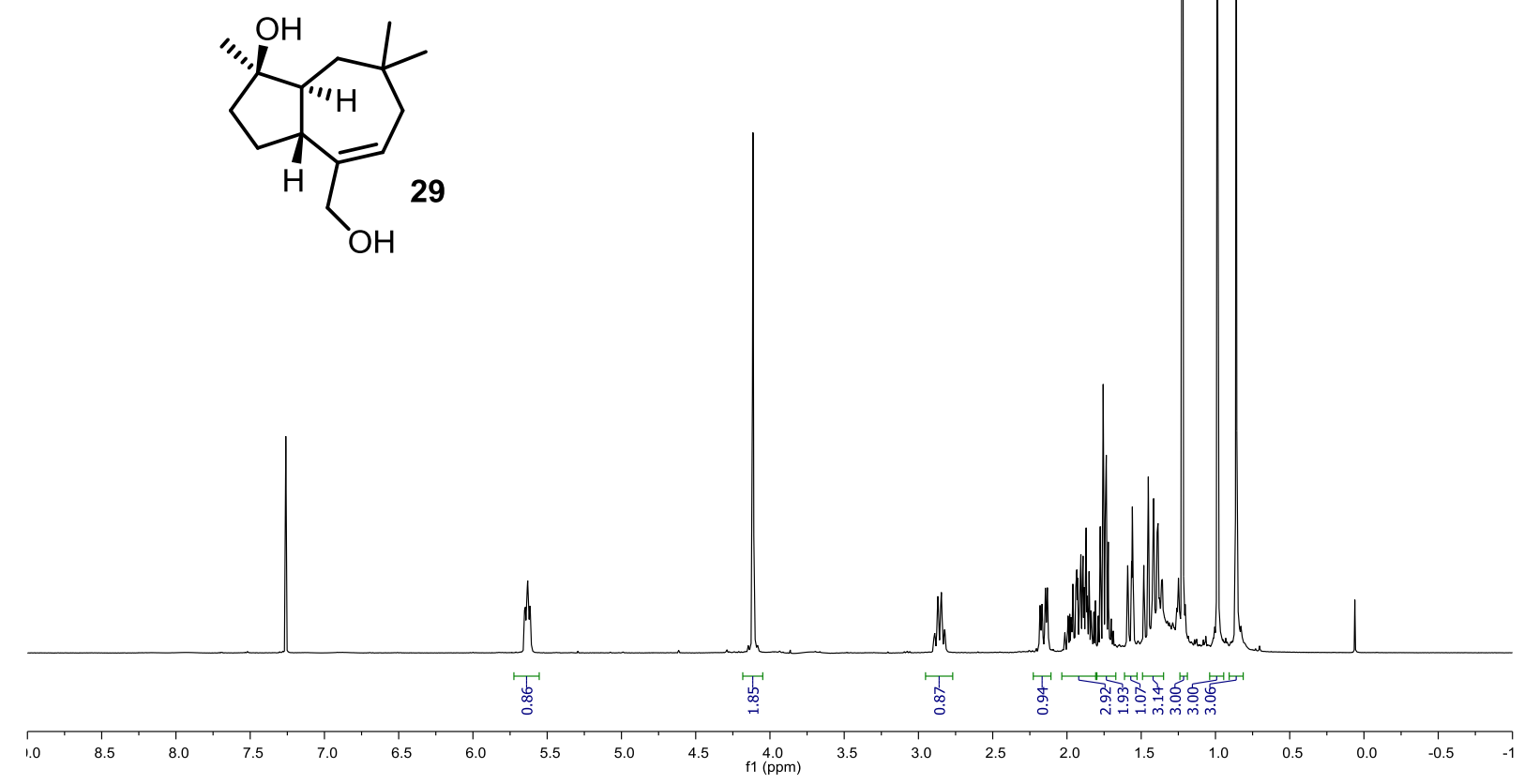

APT-NMR (101 MHz, $\left.\mathrm{CDCl}_{3}\right)$ of Compound 29

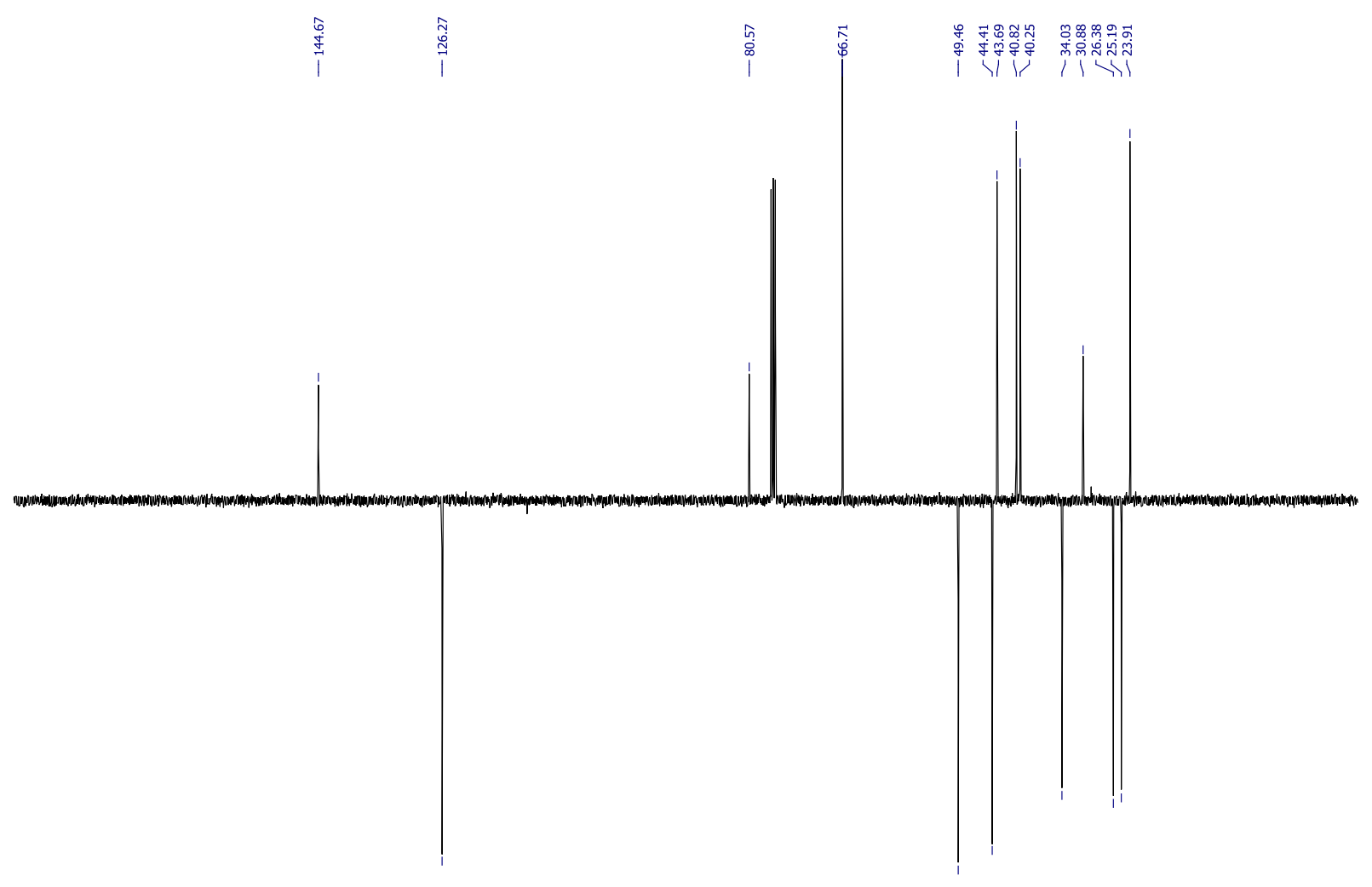

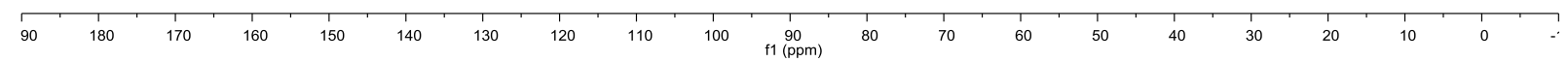


${ }^{1} \mathrm{H}$ NMR (400 MHz, (CD3)2CO) of (+)-Pyxidatol C (2)
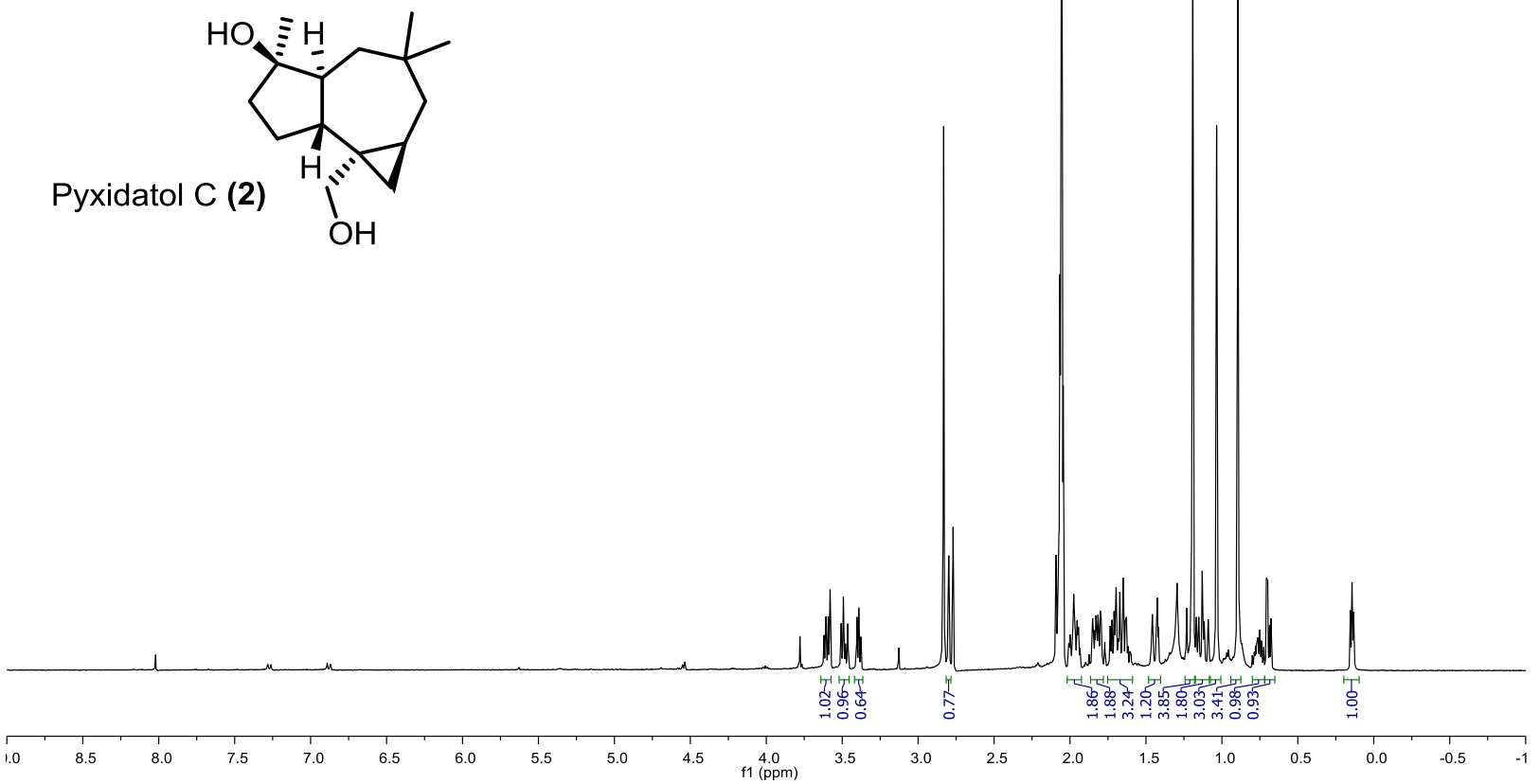

APT-NMR (101 MHz, (CD3)2CO) of (+)-Pyxidatol C (2)
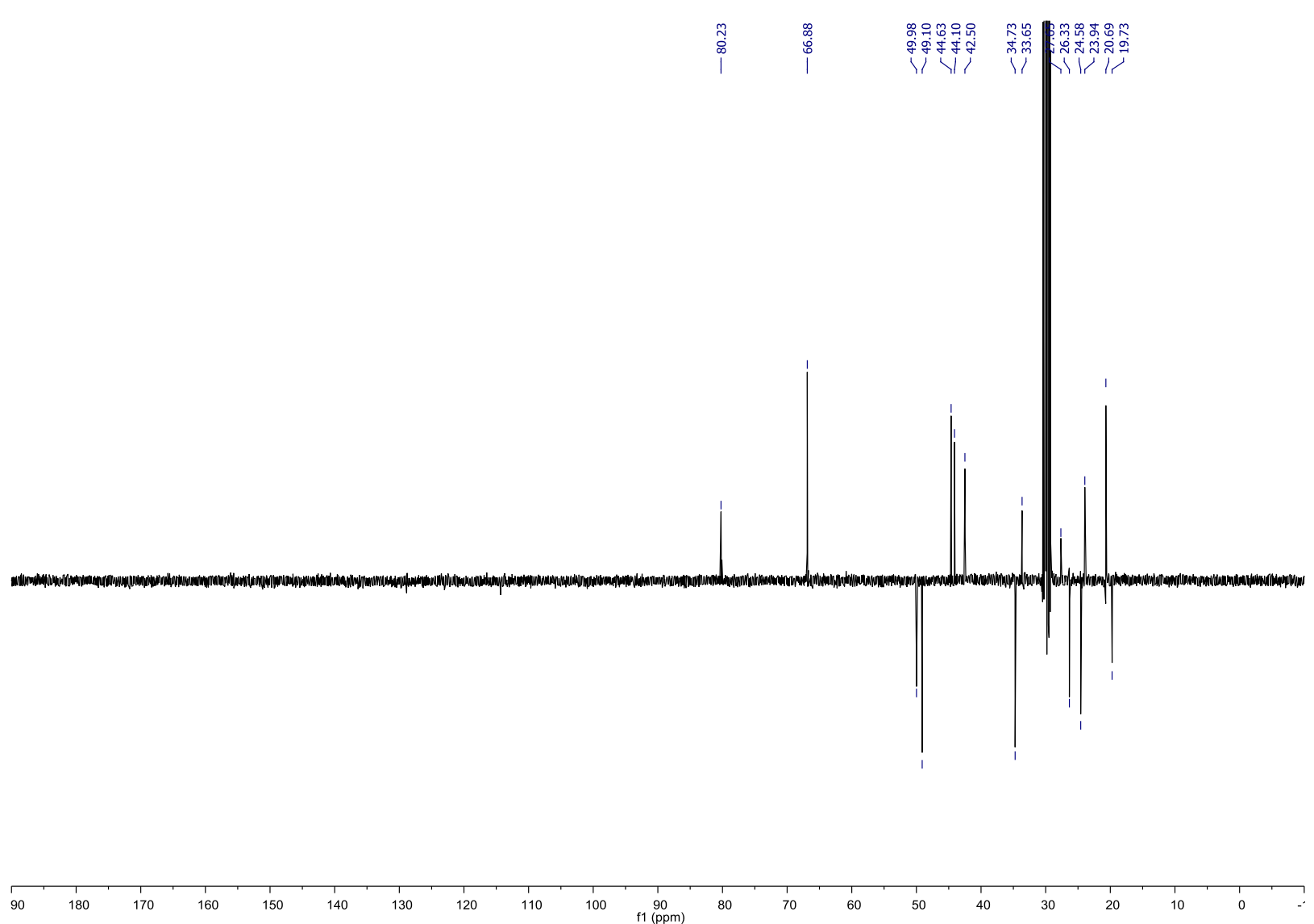